\title{
Synthesis of ( \pm )-Panduratin A and Related Natural Products Using the High Pressure Diels-Alder Reaction
}

\author{
Luke A. Pasfield, Laura de la Cruz, Junming Ho, Michelle L. Coote, Gottfried Otting, and \\ Malcolm D. McLeod*[a]
}

Panduratin A (1) is the prototypical member of a family of related cyclohexenyl natural products and is isolated as a racemic mixture from Boesenbergia rotunda, a perennial herb found in South East Asia (Figure 1). ${ }^{[1-4]}$ The plant has been used in traditional medicine to treat conditions such as diarrhea, asthma, indigestion, dysentery and many others. ${ }^{[1-4]}$ More recently studies have shown that these extracts have anti-HIV, anti-bacterial, anti-fungal and anti-tumor activity. ${ }^{[3-6]}$ Our particular interest in panduratin A (1) and its congeners stems from a report that they competitively inhibit the dengue virus NS2B-NS3 protease. ${ }^{[7,8]}$ Herein we report the synthesis of panduratin A (1) in 6 steps via a high pressure Diels-Alder reaction. This divergent sequence has also allowed for the synthesis of the related natural products 4-hydroxypanduratin A (2), panduratin H (3), panduratin I (4), nicolaioidesin $\mathrm{B}(\mathbf{5})$ and 2-hydroxyisopanduratin $\mathrm{A}(\mathbf{6}) .^{[1-9]} \mathrm{We}$ investigated the binding of 4-hydroxypanduratin $A$ (2) to the dengue virus NS2B-NS3 protease by NMR spectroscopy.

To date two syntheses of panduratin A (1) have been reported, ${ }^{[10,11]}$ both of which employed a cycloaddition reaction between a protected chalcone derivative 7 and $E$-ocimene (8) (Scheme 1). The first reported synthesis by Rahman and co-workers $^{[10]}$ in 2010 was achieved by a thermally promoted Diels-Alder reaction between chalcone derivative 7 ( $\mathrm{P}=\mathrm{MEM})$ and $E$-ocimene (8) followed by deprotection to afford a mixture of panduratin $\mathrm{A}$ and its regioisomer nicolaioidesin $\mathrm{B}$. No regioisomer ratio was reported for this mixture, nor were the regioisomers separated. In the same year Porco and co-workers ${ }^{[11]}$ employed a silver nanoparticle catalyst developed in their laboratory to facilitate the cycloaddition between a chalcone 7 (P $=\mathrm{Ac})$ and $E$-ocimene (8). This methodology afforded the adduct leading to panduratin A (1) with a small amount of the exo isomer corresponding to the isomeric natural product nicolaioidesin A.

[a] L. A. Pasfield, L. de la Cruz, Dr. J. Ho, Prof. M. L. Coote, Prof. G. Otting, Dr. M. D. McLeod

Research School of Chemistry

Australian National University

Canberra ACT 0200 (Australia)

Fax: +61261258114

E-mail:malcolm.mcleod@anu.edu.au

Supporting information for this article is available on the WWW under http://dx.doi.org/10.1002/ajoc.201 xxxxxx.

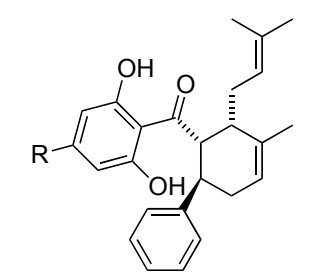

$1(\mathrm{R}=\mathrm{OMe})$ panduratin $\mathrm{A}$ $2(\mathrm{R}=\mathrm{OH})$ 4-hydroxypanduratin $\mathrm{A}$<smiles>COC(=O)[C@H]1[C@@H](CC=C(C)C)C(C)=CC[C@H]1c1ccccc1</smiles>

3 panduratin $\mathrm{H}$

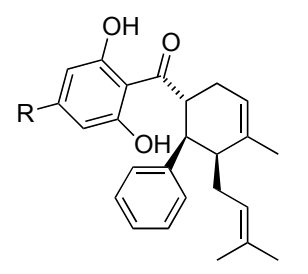

$5(\mathrm{R}=\mathrm{OMe})$ nicolaioidesin $\mathrm{B}$

$6(\mathrm{R}=\mathrm{OH})$ 2-hydroxyisopanduratin A

Figure 1. Panduratin A (1) and related natural products.<smiles>COc1cc(O)c(C(=O)C=Cc2ccccc2)c(O)c1</smiles><smiles>C=CC(C)=CCC(C)C</smiles>
1) cycloaddition 2) deprotection<smiles>[M]OC(=O)/C=C/c1ccccc1</smiles>
9<smiles>C=CC(C)=CCC=C(C)C</smiles>
addition

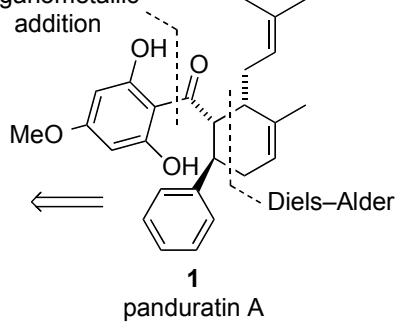

Scheme 1. Rahman ${ }^{[10]}$ and Porco ${ }^{[1]]}$ synthetic strategy and retrosynthetic analysis for this work.

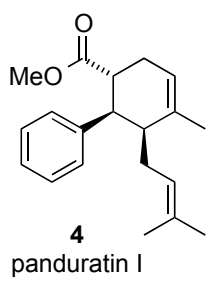

panduratin I 
These efforts serve to highlight two key issues encountered in synthetic strategies targeting the panduratin natural product family. Firstly, the requisite chalcones are typically poor dienophiles in the Diels-Alder reaction due to their high level of substitution and moderate activation and require forcing conditions or special techniques to achieve high yields. Secondly, the diene $E$-ocimene (8) is known to be sensitive to Brønsted and Lewis acids resulting in polymerization and alkene isomerization. ${ }^{[12]} \mathrm{We}$ instead envisaged an early stage high pressure Diels-Alder reaction ${ }^{[13]}$ to construct the cyclohexene core employing a sterically less encumbered and more active dienophile such as methyl cinnamate 9 with $E$-ocimene (8), followed by the construction of the phenylmethanone sidechain through organometallic addition (Scheme 1). This approach allows for late-stage access to different members of the panduratin family and is modular thereby providing avenues for the synthesis of analogues through the variation of building blocks.

The synthesis of panduratin A (1) began with the Diels-Alder cycloaddition of methyl cinnamate $\mathbf{9}$ and $E$-ocimene $(\mathbf{8}$, see Supporting Information) performed under high pressure conditions (19 kbar) to afford the regioisomeric panduratin $\mathrm{H} \mathrm{(3)}$ and $I$ (4) in a 1:2.9 ratio (Scheme 2). Employing thermal conditions or Lewis acids failed to afford the Diels-Alder

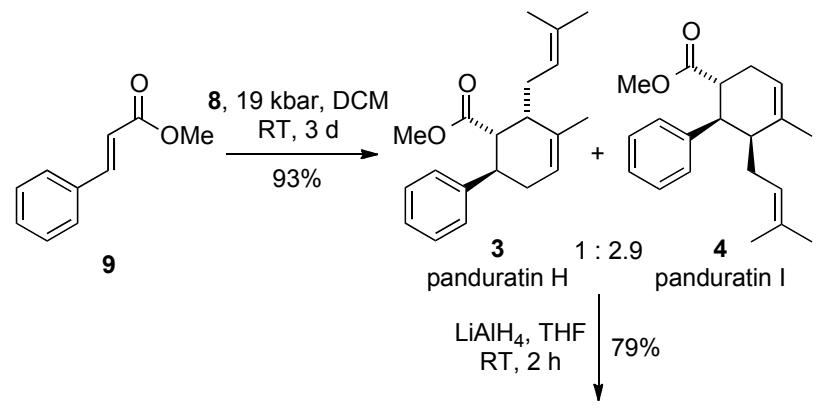<smiles>CC(C)=CC[C@H]1C(C)=CCC(c2ccccc2)[C@@H]1C=O</smiles>

12<smiles>CC(C)=CC[C@H]1C(C)=CC[C@H](CO)[C@H]1c1ccccc1</smiles>

10

11

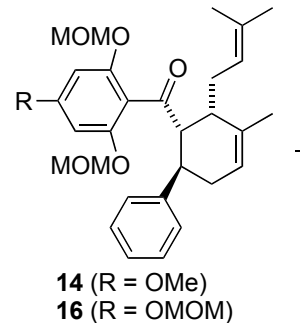

$16(\mathrm{R}=\mathrm{OMOM})$

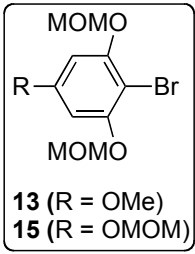

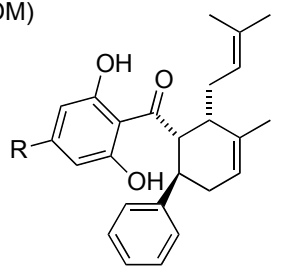

$1(\mathrm{R}=\mathrm{OMe})$ panduratin $\mathrm{A}$ $2(\mathrm{R}=\mathrm{OH})$ 4-hydroxypanduratin $\mathrm{A}$

Scheme 2. Synthesis of panduratin A (1) and 4-hydroxypanduratin A (2)

adduct. A combination of flash chromatography and HPLC separation afforded pure regioisomers that conformed to the reported literature data. ${ }^{[4]}$ In both isomers the orientation of the prenyl sidechain was cis to the adjacent ring substituent. Hydride reduction gave the alcohol products $\mathbf{1 0}$ and $\mathbf{1 1}$ that were readily separated by flash column chromatography, thus providing intermediates with the correct relative stereochemistry for the synthesis of panduratin A (1) and regioisomeric natural products. Subsequent oxidation of alcohol 10 with Dess-Martin periodinane provided aldehyde $\mathbf{1 2}$ as the branching point towards either panduratin A (1) or 4-hydroxypanduratin A (2). Addition of the organolithium reagent derived from bromobenzene $\mathbf{1 3}$ followed by Dess-Martin periodinane oxidation gave MOM protected ketone 14. Removal of the MOM groups with acidic methanol cleanly afforded panduratin A (1). Using alternate bromobenzene $\mathbf{1 5}$ and following the same synthetic sequence via ketone $\mathbf{1 6}$ gave 4-hydroxypanduratin A (2) in a yield of $52 \%$ over 3 steps. The spectroscopic data for panduratin A (1) and 4-hydroxypanduratin A (2) agreed with that of literature..$^{[1,2]}$

To further explore the influence of structure on cycloaddition regioselectivity, various dienophiles 17 with differing electronic and steric attributes were paired with $E$-ocimene $(\mathbf{8})$ in the high pressure Diels-Alder reaction (Table 1). After reaction the products were reduced to the corresponding alcohol or aldehyde which were easily separated and purified by flash column chromatography. In all cases the reactions afforded regioisomer $\mathbf{B}$ as the major product. Stronger activating groups such as the aldehyde or nitrile provided slightly higher ratios favouring regioisomer B. However, substitution of the ethyl ester for the more electron withdrawing 2,2,2-trifluoroethyl ester, a strong inductive electron withdrawing group, provided Diels-Alder adducts in a similar ratio. ${ }^{[14,15]}$

Table 1. High pressure Diels-Alder reactions of different dienophiles 17 with E-ocimene (8).

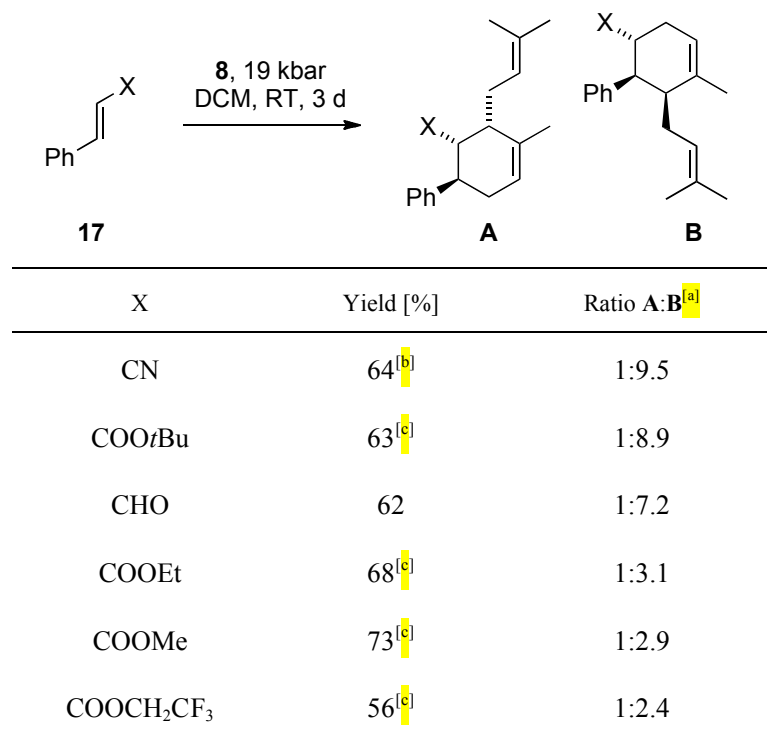

[a] Ratio of regioisomers determined by $400 \mathrm{MHz}{ }^{1} \mathrm{H}$ NMR integration of the crude reaction mixture. [b] After reduction to aldehyde. [c] After reduction to alcohol.

The regiochemical outcome of the Diels-Alder cycloaddition between methyl cinnamate (9) with $E$-ocimene (8) was in accord with an analysis based on computationally-derived frontier 
molecular orbitals (Figure 2). Alignment of the larger diene HOMO $\left(2 \mathrm{P}_{\mathrm{z}}\right.$ atomic) coefficient with that of the corresponding dienophile LUMO coefficient predicts formation of ester $\mathbf{4}$ as the major regioisomer. ${ }^{[16]}$ Orbital coefficients also predict the correct regiochemical outcomes for the other compounds studied in Table 1 (see the Supporting Information for further details).

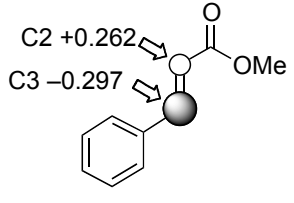

9

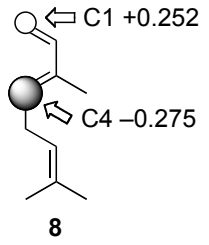

Figure 2. Atomic orbital coefficients of the $2 \mathrm{p}$ orbitals of methyl cinnamate (9), and $E$-ocimene (8) calculated at the B3LYP/6-31G(d) level of theory (see computational methods in the Supporting Information for further details).

Employing cinnamaldehyde (18) in the high pressure DielsAlder reaction afforded a separable 1:7.2 mixture of the two Diels-Alder regioisomers 12 and $\mathbf{1 9}$ (Scheme 3). Addition of the organolithium reagent derived from bromobenzene $\mathbf{1 3}$ to aldehyde 19 followed by oxidation afforded ketone $\mathbf{2 0}$ in $69 \%$ over two steps. Deprotection gave nicolaioidesin B (5) in $69 \%$ yield. $^{[9]}$ Using alternate bromobenzene $\mathbf{1 5}$ and following the same synthetic sequence via ketone $\mathbf{2 1}$ gave 4-hydroxyisopanduratin A (6) in a yield of $50 \%$ over 3 steps. The spectroscopic data for nicolaioidesin B (5) and 4-hydroxyisopanduratin A (6) agreed with that of literature. ${ }^{[3,9]}$

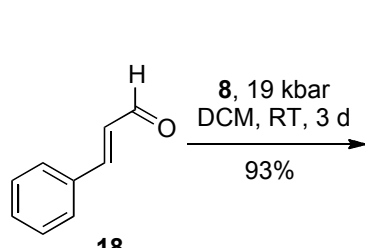

18<smiles>CC(C)=CC[C@H]1C(C)=CC[C@H](c2ccccc2)[C@H]1C=O</smiles>

12

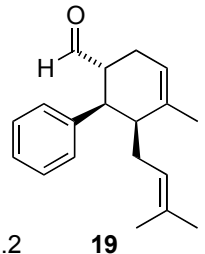

19

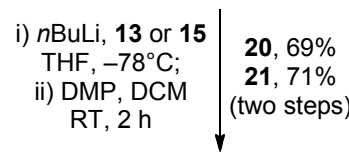

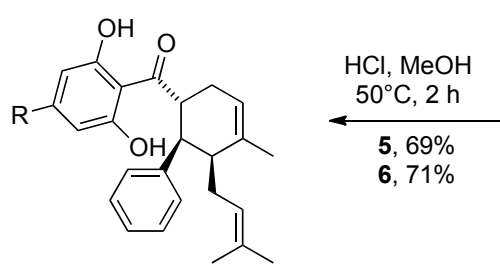

$5(\mathrm{R}=\mathrm{OMe})$ nicolaioidesin $\mathrm{B}$

$6(\mathrm{R}=\mathrm{OH})$ 4-hydroxyisopanduratin $\mathrm{A}$

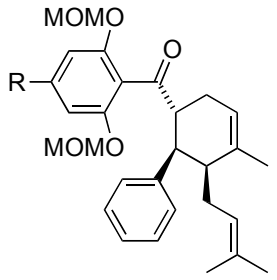

$20(\mathrm{R}=\mathrm{OMe})$

$21(\mathrm{R}=\mathrm{OMOM})$
Scheme 3. Synthesis of nicolaioidesin B (5) and 4-hydroxyisopanduratin A (6).

The dengue virus NS2B-NS3 protease is crucial for viral replication and presents a target for the treatment of dengue virus infections. The hydrophilic NS2B fragment acts as a cofactor essential for full proteolytic activity. Recent NMR studies in association with pseudocontact shifts derived from paramagnetic lanthanide tags have shown that binding of an inhibitor at the substrate-binding site can trigger a large conformational change of the NS2B cofactor, shifting the protein structure from an open to a closed conformation. ${ }^{[17]}$ Crystal structures of the NS2B-NS3 protease with bound inhibitors show that in the closed conformation, which is also thought to be the active conformation, NS2B lines the substrate binding site. ${ }^{[18]}$

Both panduratin A (1) $\left(K_{\mathrm{i}}=25 \quad \pm \quad 8 \quad \mu \mathrm{M}\right)$ and 4-hydroxypanduratin A (2) $\left(K_{\mathrm{i}}=21 \pm 6 \mu \mathrm{M}\right)$ have been reported, based on enzyme kinetic data, to inhibit the dengue virus NS2BNS3 protease competitively, identifying them as important natural product lead structures. ${ }^{[7,8]}$ Thus NMR spectroscopy suggested itself as a suitable method to further define the nature of binding of these natural products at the NS2B-NS3 protease. To test whether panduratins can induce a closed conformation we recorded ${ }^{15} \mathrm{~N}$-HSQC spectra of the uniformly ${ }^{15} \mathrm{~N}$-labelled NS2B$\mathrm{NS} 3$ protease in the presence and absence of the compounds. No changes to the spectrum NS2B-NS3 protease were observed in the presence of panduratin A (1) or 4-hydroxypanduratin A (2), in contrast to the response to previously reported low molecular weight competitive inhibitors. ${ }^{[17]}$ Previous NMR and X-ray data showed, that binding of inhibitors at the substrate binding site can stabilize a closed conformation of the protease that resembles the enzymatically active state. ${ }^{[17,18]}$ The NMR spectra obtained in the presence of either panduratin A (1) or 4-hydroxy-panduratin A (2) showed no evidence for such a conformational change. In our hands, panduratin A (1) and 4-hydroxypanduratin A (2) also proved to be poorly soluble in aqueous media. Addition of either of the two compounds from DMSO stock solutions to the protein sample caused precipitation of the panduratins. Panduratin A (1) precipitated even at a target concentration of $100 \mu \mathrm{M}$ in $15 \%$ aqueous DMSO. These results highlight that some caution is warranted in adopting panduratin A (1) and 4-hydroxypanduratin A (2) as lead structures for the development of dengue virus NS2B-NS3 protease inhibitors.

In conclusion we have completed the synthesis of panduratin A (1) and five related natural products 2-6 using a high pressure Diels-Alder reaction as the key step. This flexible route provides rapid access to various members of the natural product family and is amenable to analogue synthesis. However, attempts to monitor the binding of panduratin A (1) and 4-hydroxypanduratin A (2) to the dengue virus NS2-NS3 protease by NMR failed to show the characteristic changes in protein conformation that have been observed for other known competitive inhibitors.

\section{Acknowledgements}

We thank the Australian Research Council for financial support. MLC thanks the National Facility of the National Computational Infrastructure for supercomputing time.

Keywords: dengue virus $\bullet$ high-pressure chemistry • natural products $\bullet$ protein NMR $\bullet$ total synthesis

[1] P. Tuntiwachwuttikul, O. Pancharoen, V. Reutrakul, L. T. Byrne, Aust. J. Chem. 1984, 37, 449-453.

[2] G. Trakoontivakorn, K. Nakahara, H. Shinmoto, M. Takenaka, M. OnishiKameyama, H. Ono, M. Yoshida, T. Nagata, T. Tsushida, J. Agric. Food Chem. 2001, 49, 3046-3050.

[3] N. N. Win, S. Awale, H. Esumi, Y. Tezuka, S. Kadota, J. Nat. Prod. 2007, 70, $1582-1587$. 
[4] N. N. Win, S. Awale, H. Esumi, Y. Tezuka, S. Kadota, Chem. Pharm. Bull. 2008, 56, 491-496.

[5] S. Cheenpracha, C. Karalai, C. Ponglimanont, S. Subhadhirasakul, S. Tewtrakul, Bioorg. Med. Chem. 2006, 14, 1710-1714.

[6] I. Deb Majumdar, A. Devanabanda, B. Fox, J. Schwartzman, H. Cong, J. A. Porco Jr., H. C. Weber, Biochem. Biophys. Res. Commun. 2011, 416, 397402.

[7] T. S. Kiat, R. Pippen, R. Yusof, H. Ibrahim, N. Khalid, N. A. Rahman, Bioorg. Med. Chem. Lett. 2006, 16, 3337-3340.

[8] A. J. Stevens, M. E. Gahan, S. Mahalingam, P. A. Keller, J. Med. Chem. 2009, 52, 7911-7926.

[9] J.-Q. Gu, E. J. Park, J. S. Vigo, J. G. Graham, H. H. S. Fong, J. M. Pezzuto, A. D. Kinghorn, J. Nat. Prod. 2002, 65, 1616-1620.

[10] C. F. Chee, I. Abdullah, M. J. C. Buckle, N. A. Rahman, Tetrahedron Lett. 2010, 51, 495-498.

[11] H. Cong, C. F. Becker, S. J. Elliott, M. W. Grinstaff, J. A. Porco Jr., J. Am. Chem. Soc. 2010, 132, 7514-7518.
[12] G. Majetich, Y. Zhang, J. Am. Chem. Soc. 1994, 116, 4979-4980.

[13] K. Matsumoto, H. Hamana, H. Iida, Helv. Chim. Acta 2005, 88, 2033-2234.

[14] T. Kan, Y. Ohfune, Tetrahedron Lett. 1995, 36, 943-946.

[15] Y. Ohfune, T. Kan, T. Nakajima, Tetrahedron 1998, 54, 5207-5224.

[16] K. N. Houk, J. Am. Chem. Soc. 1973, 95, 4092-4094.

[17] L. de la Cruz, T. H. D. Nguyen, K. Ozawa, J. Shin, B. Graham, T. Huber, G. Otting, J. Am. Chem. Soc. 2011, 133, 19205-19215.

[18] C. G. Noble, C. C. Seh, A. T. Chao, P. Y. Shi, J. Virol. 2012, 86, 438-446.

Received: ((will be filled in by the editorial staff)) Revised: ((will be filled in by the editorial staff)) Published online: ((will be filled in by the editorial staff)) 
Entry for the Table of Contents

\section{Natural Products}

L. A. Pasfield, L. de la Cruz, J. Ho, M. L. Coote, G. Otting,

M. D. McLeod* ..... Page - Page

Synthesis of ( \pm )-Panduratin $A$ and Related Natural Products Using the High Pressure Diels-Alder Reaction

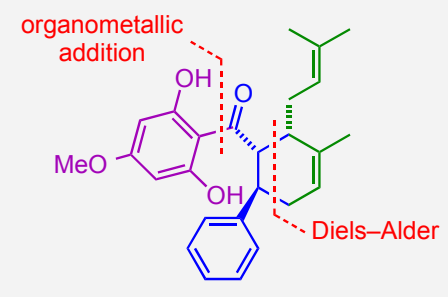

panduratin A (1)
Under Pressure: Panduratin A (1) has been synthesized in 6 steps via a high pressure Diels-Alder reaction. This divergent sequence has also allowed for the synthesis of 4hydroxypanduratin A (2), panduratin $\mathrm{H}$ (3), panduratin I (4), nicolaioidesin $\mathrm{B}$ (5) and 2-hydroxyisopanduratin A (6). The binding of panduratin A (1) to the dengue virus NS2B-NS3 protease has been investigated by NMR spectroscopy. 
Synthesis of ( \pm )-Panduratin A and Related Natural Products Using the High Pressure Diels-Alder Reaction

Luke A. Pasfield, Laura de la Cruz, Junming Ho, Michelle L. Coote, Gottfried Otting, and Malcolm D. McLeod*

Research School of Chemistry, Australian National University, Canberra, ACT 0200, Australia

Fax: +61 26125 8114; E-mail: malcolm.mcleod@anu.edu.au 


\section{Contents}

$\begin{array}{ll}\text { Chemistry Experimental } & \mathrm{S} 3\end{array}$

${ }^{1} \mathrm{H}$ and ${ }^{13} \mathrm{C}$ NMR Comparisons $\quad$ S18

$\begin{array}{ll}\text { Computational Details } & \text { S30 }\end{array}$

B3LYP/6-31+G(d) Optimized Geometries $\quad$ S30

$\begin{array}{ll}\text { Binding Studies by NMR Spectroscopy } & \text { S34 }\end{array}$

$\begin{array}{ll}\text { References } & \text { S35 }\end{array}$

$\begin{array}{ll}{ }^{1} \mathrm{H} \text { and }{ }^{13} \mathrm{C} \text { NMR Spectra } & \text { S37 }\end{array}$ 


\section{Chemistry Experimental}

\section{General}

Infrared absorption (IR) spectra were obtained using with a Perkin-Elmer Spectrum One FTIR spectrometer. Compounds were prepared as a thin film between $0.5 \mathrm{~cm}$ sodium chloride plates. Absorption maxima $\left(v_{\max }\right)$ are expressed in wavenumbers $\left(\mathrm{cm}^{-1}\right) .{ }^{1} \mathrm{H}$ Nuclear magnetic resonance spectra were recorded using a Varian Mercury 300 (300 MHz), Varian Mercury 400 (400 MHz) or Bruker $800(800 \mathrm{MHz})$ spectrometer at $25^{\circ} \mathrm{C}$, and are recorded in parts per million (ppm) downfield shift from tetramethylsilane $\left(\delta_{\mathrm{TMS}}=0\right)$, using residual chloroform $(\delta 7.26)$ or methanol $(\delta$ 3.31) solvent as internal reference. The data is reported as chemical shift $\left(\delta_{\mathrm{H}}\right)$, relative integral, multiplicity $(\mathrm{s}=$ singlet, $\mathrm{br}=$ broad, $\mathrm{d}=$ doublet, $\mathrm{t}=$ triplet, $\mathrm{q}=$ quartet, $\mathrm{m}=$ multiplet $)$, coupling constant $(J \mathrm{~Hz})$, and assignment. ${ }^{13} \mathrm{C}$ Nuclear magnetic resonance spectra were recorded using a Varian Mercury $300(75 \mathrm{MHz})$ or Varian Mercury $400(100 \mathrm{MHz})$ spectrometer at $25^{\circ} \mathrm{C}$ with complete proton decoupling. Data is expressed in parts per million (ppm) downfield relative to tetramethylsilane $\left(\delta_{\mathrm{TMS}}=0\right)$ using deuterated chloroform $(\delta 77.00)$ solvent as an internal reference and is reported as chemical shift $\left(\delta_{\mathrm{C}}\right)$. High and low resolution electron impact ionization (EI) mass spectra were recorded using a Micromass VG Autospec mass spectrometer. Low and high resolution electrospray ionization (ESI) mass spectra were recorded using a Micromass ZMD LR mass spectrometer or a Waters LCT Premier XE mass spectrometer respectively. Preparative HPLC was performed on a Waters preparative HPLC system consisting of a 600E solvent delivery system, in-line degasser and 2996 diode array detector. Samples were injected manually via a Rheodyne $7725 \mathrm{i}$ injection valve fitted with a $5 \mathrm{~mL}$ loop and separated on a Waters XBridge C18 $5 \mu \mathrm{m} 150$

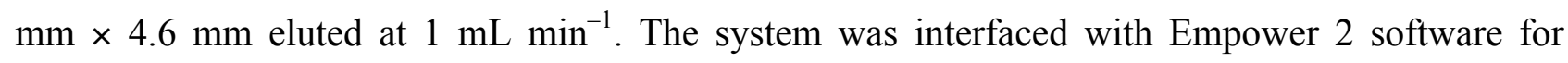
instrument control and data acquisition. Analytical thin layer chromatography (TLC) was performed using $0.2 \mathrm{~mm}$ thick aluminum backed pre-coated silica gel plates (Merck Kieselgel 60 F254). Flash chromatography was carried out using Merck Kieselgel 60 (230-400 mesh ASTM), under a positive pressure of nitrogen. Solvent compositions were mixed v/v as specified. High pressure reactions were carried out in a PSIKA high pressure reactor. All solvents and reagents were purified according to standard literature procedures. ${ }^{[1]}$

\section{3-Methyl-2-(3-methylbut-2-en-1-yl)-2,5-dihydrothiophene 1,1-dioxide (22) ${ }^{[2]}$}

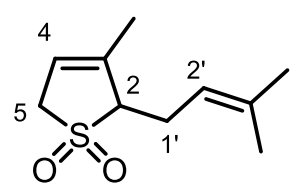

3-Methyl sulfolene (3.46 g, $26.2 \mathrm{mmol})$, prenyl bromide (1.53 mL, $13.1 \mathrm{mmol})$ and tetrahydrofuran $(50 \mathrm{~mL})$ were added to a flask and under a nitrogen atmosphere and cooled to $-95^{\circ} \mathrm{C}$ with stirring. 
LHMDS (26.2 mL, $1 \mathrm{M}, 26.2 \mathrm{mmol})$ in tetrahydrofuran was then slowly added over $1 \mathrm{~min}$ and the reaction allowed to stir for a further $1 \mathrm{~min}$. Quenching with a saturated solution of ammonium chloride $(30 \mathrm{~mL})$ was then followed by extraction with diethyl ether $(3 \times 20 \mathrm{~mL})$. The combined organic layers were then dried over magnesium sulfate, filtered, concentrated under reduced pressure and purified via silica gel chromatography using diethyl ether : hexane (2:3) to afford 3methyl-2-(3-methylbut-2-en-1-yl)-2,5-dihydrothiophene 1,1-dioxide 22 as a clear yellow oil (2.15 g, 82\%); $\mathbf{R}_{\mathbf{f}}$ (diethyl ether:hexane 2:3) 0.19; ${ }^{1} \mathbf{H}-\mathbf{N M R}\left(400 \mathrm{MHz}, \mathrm{CDCl}_{3}\right) \delta 5.68(1 \mathrm{H}, \mathrm{m}, \mathrm{H} 4), 5.21$ (1H, m, H2'), 3.73-3.60 (2H, m, H5), 3.50 (1H, t, $J=6.0$ Hz, H2), 2.61-2.49 (2H, m, H1'), 1.85 (3H, s, $\left.\mathrm{CH}_{3}\right), 1.72\left(3 \mathrm{H}, \mathrm{s}, \mathrm{CH}_{3}\right), 1.66\left(3 \mathrm{H}, \mathrm{s}, \mathrm{CH}_{3}\right) ;{ }^{13} \mathbf{C}-\mathbf{N M R}\left(100 \mathrm{MHz}, \mathrm{CDCl}_{3}\right) \delta 138.6,135.3,118.3$, 117.0, 67.2, 55.6, 26.4, 25.7, 18.1, 17.8; IR (thin film) $v_{\max }=2970$ (m), 2920 (m), 2856 (m), 1730 (w), 1671 (w), 1442 (m) 1380 (m), 1303 (s) $\mathrm{cm}^{-1}$; LRMS (EI $\left.{ }^{+}\right) \mathrm{m} / z=200\left(\mathrm{M}^{+\bullet}, 10 \%\right), 135([\mathrm{M}-$ $\left.\left.\mathrm{SO}_{2}-\mathrm{H}\right]^{+\bullet}, 100 \%\right)$; HRMS $\left(\mathrm{EI}^{+}\right)$calcd for $\mathrm{C}_{10} \mathrm{H}_{16} \mathrm{O}_{2} \mathrm{~S}\left(\mathrm{M}^{+\bullet}\right)$ 200.0871, found 200.0873, calcd for $\mathrm{C}_{10} \mathrm{H}_{15}\left(\left[\mathrm{M}-\mathrm{SO}_{2} \mathrm{H}\right]^{+\bullet}\right)$ 135.1174, found 135.1172 .

\section{$\left(\right.$ E)-Ocimene (8) ${ }^{[2]}$}

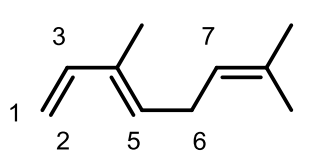

3-Methyl-2-(3-methylbut-2-en-1-yl)-2,5-dihydrothiophene 1,1-dioxide 22 (403 mg, $2.01 \mathrm{mmol}$ ) and pyridine $(20 \mathrm{~mL})$ were added to a flask and heated at reflux for 6 hours with stirring. After cooling to room temperature the reaction was extracted with $30-40$ petroleum spirits $(20 \mathrm{~mL})$ and washed with $2 \mathrm{M}$ hydrochloric acid solution $(4 \times 20 \mathrm{~mL})$. The petroleum spirit layer was then washed through a silica gel plug and concentrated under reduced pressure to give $E$-ocimene $\mathbf{8}$ as a clear colorless oil (222 mg, 81\%); $\mathbf{R}_{\mathbf{f}}$ (diethyl ether:hexane 2:3) $0.75 ;{ }^{1} \mathbf{H}-\mathbf{N M R}\left(300 \mathrm{MHz}, \mathrm{CDCl}_{3}\right) \delta 6.37$ (1H, dd, $J=17.4 \& 11.1 \mathrm{~Hz}, \mathrm{H} 3), 5.46$ (1H, t, $J=7.8 \mathrm{~Hz}, \mathrm{H} 5), 5.16-5.07$ (2H, m, H2 \& H7), 4.94 $(1 \mathrm{H}, \mathrm{d}, J=10.5 \mathrm{~Hz}, \mathrm{H} 1), 2.84(2 \mathrm{H}, \mathrm{t}, J=7.5 \mathrm{~Hz}, \mathrm{H} 6), 1.77\left(3 \mathrm{H}, \mathrm{s}, \mathrm{CH}_{3}\right), 1.71\left(3 \mathrm{H}, \mathrm{s}, \mathrm{CH}_{3}\right), 1.65$ $\left(3 \mathrm{H}, \mathrm{s}, \mathrm{CH}_{3}\right) ;{ }^{13} \mathbf{C}-\mathbf{N M R}\left(75 \mathrm{MHz}, \mathrm{CDCl}_{3}\right) \delta 141.6,133.8,132.3,131.9,122.3,110.7,27.4,25.8$, 17.8, 11.7; IR (thin film) $v_{\max }=2975$ (s), 2926 (s), 2857 (m), 1789 (w), 1640 (m), 1606 (m), 1448 (m), $1376(\mathrm{~m}) \mathrm{cm}^{-1}$; LRMS $\left(\mathrm{EI}^{+}\right) \mathrm{m} / z=136\left(\mathrm{M}^{+\bullet}, 45 \%\right), 93(100 \%)$. 
( \pm )-Panduratin $\mathrm{H}(3)$ and $( \pm)$-panduratin I (4) ${ }^{[3]}$
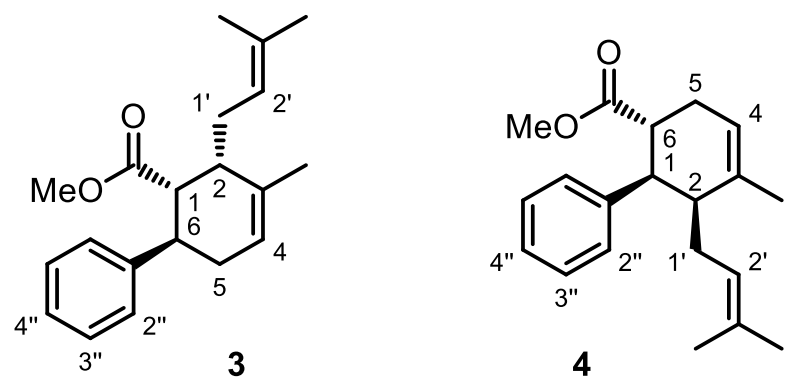

Methyl cinnamate 9 (601 mg, $3.71 \mathrm{mmol}), E$-ocimene 8 (2.02 g, $14.8 \mathrm{mmol})$ and dichloromethane $(2 \mathrm{~mL})$ were added to a pressure vessel, sealed and pressurized to $19 \mathrm{kbar}$ for 3 days. The reactions was then diluted with dichloromethane $(20 \mathrm{~mL})$, evaporated on to silica and purified by column chromatography using diethyl ether : hexane (1:9) and then preparative HPLC on Waters Xbridge C18, 60\% acetonitrile, $40 \%$ water: to afford $\left(\mathrm{R}_{\mathrm{t}} 31.1 \mathrm{~min}\right)( \pm)$-panduratin $\mathrm{H} 3$ as a clear colorless oil (257 mg, 23\%); $\mathbf{R}_{\mathbf{f}}$ (diethyl ether:hexane 1:4) 0.44; ${ }^{1} \mathbf{H}-\mathbf{N M R}\left(400 \mathrm{MHz}, \mathrm{CDCl}_{3}\right) \delta 7.26(2 \mathrm{H}, \mathrm{m}$, H3"), 7.20-7.13 (3H, m, H2"\& H4"), 5.42 (1H, br s, H4), 5.06 (1H, m, H2'), 3.40 (3H, s, $\left.\mathrm{OCH}_{3}\right)$, 3.19 (1H, ddd, $J=12.0,10.4 \& 6.4 \mathrm{~Hz}, \mathrm{H} 6), 3.08(1 \mathrm{H}, \mathrm{dd}, J=12.4,4.8 \mathrm{~Hz}, \mathrm{H} 1), 2.45$ (1H, dd, $J=$

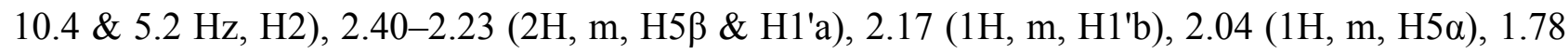
$\left(3 \mathrm{H}, \mathrm{s}, \mathrm{CH}_{3}\right), 1.67\left(3 \mathrm{H}, \mathrm{s}, \mathrm{CH}_{3}\right), 1.60\left(3 \mathrm{H}, \mathrm{s}, \mathrm{CH}_{3}\right) ;{ }^{13} \mathbf{C}-\mathbf{N M R}\left(100 \mathrm{MHz}, \mathrm{CDCl}_{3}\right) \delta 173.8,145.9$, 136.3, 132.0, 128.3 127.1, 126.0, 123.8, 121.0, 51.0, 49.1, 42.6, 37.1, 35.1, 29.2, 25.9, 22.6, 17.9; IR (thin film) $v_{\max }=2966$ (m), 2949 (m), 2914 (m), 1741 (vs), 1494 (w), 1434 (m), 1301 (w), 1157(s); LRMS $\left(\mathrm{EI}^{+}\right) \mathrm{m} / z=298\left(\mathrm{M}^{+\bullet}, 60 \%\right), 169(100 \%)$; HRMS (EI $)$ calcd for $\mathrm{C}_{20} \mathrm{H}_{26} \mathrm{O}_{2}\left(\mathrm{M}^{+\bullet}\right)$ 298.1933, found 298.1935. A further fraction from flash column chromatography gave $( \pm)$ panduratin I 4 as a colorless oil (772 mg, 70\%); $\mathbf{R}_{\mathbf{f}}$ (diethyl ether:hexane 1:4) 0.39; ${ }^{\mathbf{H}} \mathbf{H}-\mathbf{N M R}$ (400 $\left.\mathrm{MHz} \mathrm{CDCl}_{3}\right) \delta 7.26$ (2H, m, H3"), 7.19-7.14 (3H, m, H2"\& H4"), 5.45 (1H, br s, H4), 4.81 (1H, m, H2'), $3.49\left(3 \mathrm{H}, \mathrm{s}, \mathrm{OCH}_{3}\right), 3.33(1 \mathrm{H}, \mathrm{dd}, J=11.2 \& 5.2 \mathrm{~Hz}, \mathrm{H} 1), 3.18(1 \mathrm{H}, \mathrm{ddd}, J=11.2,9.6$ \& 6.4 Hz, H6), 2.44 (1H, m, H5ß), 2.32 (1H, m, H5 ), 2.23 (1H, dd, $J=10.8 \& 5.2$ Hz, H2), 1.99-1.86 $\left(2 \mathrm{H}, \mathrm{m}, \mathrm{H1} 1^{\prime}\right), 1.74\left(3 \mathrm{H}, \mathrm{s}, \mathrm{CH}_{3}\right), 1.57\left(3 \mathrm{H}, \mathrm{s}, \mathrm{CH}_{3}\right), 1.34\left(3 \mathrm{H}, \mathrm{s}, \mathrm{CH}_{3}\right) ;{ }^{13} \mathbf{C}-\mathbf{N M R}\left(100 \mathrm{MHz}, \mathrm{CDCl}_{3}\right)$ $\delta 176.5,142.3,137.6,131.0,128.2,128.1,126.1,123.4,119.5,51.5,46.3,44.5,39.9,29.5,27.6$, 25.8, 22.9, 17.7; IR (thin film) $v_{\max }=2966$ (m), 2950 (m), 2913 (m), 1739 (vs), 1496 (w), 1435 (m), 1265 (m), $1162(\mathrm{~s})$; LRMS $\left(\mathrm{EI}^{+}\right) \mathrm{m} / z=298\left(\mathrm{M}^{+*}, 60 \%\right), 169(100 \%)$; HRMS (EI $)$ calcd for $\mathrm{C}_{20} \mathrm{H}_{26} \mathrm{O}_{2}\left(\mathrm{M}^{+\bullet}\right)$ 298.1933, found 298.1935. 

(11)
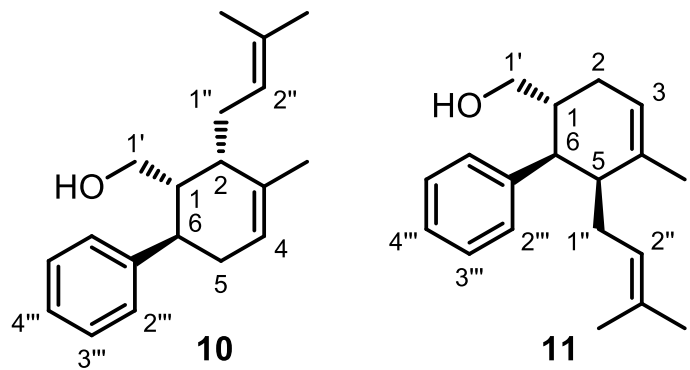

To a solution of panduratin H 3 and panduratin I 4 (251 mg, $0.841 \mathrm{mmol})$ in tetrahydrofuran (20 $\mathrm{mL})$ was added $1 \mathrm{M}$ lithium aluminum hydride in tetrahydrofuran $(3.37 \mathrm{~mL}, 3.37 \mathrm{mmol})$ dropwise over 5 minutes. After $1 \mathrm{~h}$ of stirring at room temperature the reaction was cooled to $0^{\circ} \mathrm{C}$ and ethyl acetate $(10 \mathrm{~mL})$ was added dropwise over 10 minutes. The reaction was then diluted with diethyl ether $(80 \mathrm{~mL})$ and a saturated solution of potassium sodium tartrate $(80 \mathrm{~mL})$ and stirred vigorously for 30 minutes. The diethyl ether layer was then separated and the aqueous layer re-extracted with diethyl ether $(2 \times 50 \mathrm{~mL})$. The combined organic layers were washed with brine $(50 \mathrm{~mL})$ and evaporated on to silica with purification by column chromatography using diethyl ether : hexane (1:4) affording (3-methyl-2-(3-methylbut-2-en-1-yl)-6-phenyl-3-cyclohexene-1-yl)methanol 10 as a colorless oil (45.5 mg, 20\%); $\mathbf{R}_{\mathbf{f}}$ (diethyl ether:hexane $\left.3: 7\right) \quad 0.20 ;{ }^{1} \mathbf{H}-\mathbf{N M R}\left(400 \mathrm{MHz}, \mathrm{CDCl}_{3}\right) \delta$ 7.27 (2H, m, H3'"), 7.20-7.17 (3H, t, J=8.4 Hz, H2"'\& H4"'), 5.45 (1H, br s, H4), 5.28 (1H, m, H2"), 3.38-3.26 (2H, m, H1'), 2.79 (1H, ddd, $J=10.8,10.8$ \& 6.4 Hz, H6), 2.33-2.15 (6H, m, H1, H2 H5 \& H1"), $1.78\left(3 \mathrm{H}, \mathrm{s}, \mathrm{CH}_{3}\right), 1.71\left(3 \mathrm{H}, \mathrm{s}, \mathrm{CH}_{3}\right), 1.68\left(3 \mathrm{H}, \mathrm{s}, \mathrm{CH}_{3}\right),(\mathrm{OH}$ not observed); ${ }^{13}$ C-NMR $\left(100 \mathrm{MHz}, \mathrm{CDCl}_{3}\right) \delta 145.0,137.2,132.0,128.5,127.6,126.3,124.6,121.2,63.4,45.0$, 40.5, 39.0, 34.9, 27.7, 25.8, 22.7, 18.0; IR (thin film) $v_{\max }=3350$ (m), 2963 (s), 2911 (s), $1602(\mathrm{w})$, 1452 (m), 1376 (w), 1048 (m), 757 (m); LRMS (ESI $\left.{ }^{+}\right)$m/z = $293\left([\mathrm{M}+\mathrm{Na}]^{+}, 58 \%\right), 102$ (100\%); HRMS $\left(\mathrm{ESI}^{+}\right)$calcd for $\mathrm{C}_{19} \mathrm{H}_{26} \mathrm{ONa}\left([\mathrm{M}+\mathrm{Na}]^{+}\right)$, 293.1881, found 293.1881. A second fraction afforded (4-methyl-5-(3-methylbut-2-en-1-yl)-6-phenylcyclohexa-3-en-1-yl)methanol $\mathbf{1 1}$ as a colorless oil (134 mg, 59\%); $\mathbf{R}_{\mathbf{f}}$ (diethyl ether:hexane $\left.3: 7\right)$ 0.15; ${ }^{1} \mathbf{H}-\mathbf{N M R}$ (400 MHz, $\mathrm{CDCl}_{3}$ ) $\delta 7.28$ (2H, m, H3"'), 7.20-7.16 (3H, m, H2"'\& H4"'), 5.48 (1H, s, H3), 4.90 (1H, m, H2"), 3.60 (1H, m, H1'a), 3.42 (1H, m, H1'b), 2.92 (1H, dd, $J=9.6$ \& 4.8 Hz, H6), 2.36-2.24 (2H, m, H1 \& H2a), 2.15 (1H, m, H5), 2.06-1.99 (3H, m, H2b \& H1"), $1.73\left(3 \mathrm{H}, \mathrm{s}, \mathrm{CH}_{3}\right), 1.58\left(3 \mathrm{H}, \mathrm{s}, \mathrm{CH}_{3}\right), 1.34(3 \mathrm{H}, \mathrm{s}$, $\left.\mathrm{CH}_{3}\right),\left(\mathrm{OH}\right.$ not observed); ${ }^{13} \mathbf{C}-\mathbf{N M R}\left(100 \mathrm{MHz}, \mathrm{CDCl}_{3}\right) \delta 142.4,137.1,130.9,129.3,128.0,126.0$, 123.6, 121.1, 66.1, 46.1, 44.1, 35.0, 28.1, 27.5, 25.8, 22.8, 17.7; IR (thin film) $v_{\max }=3326(\mathrm{~m})$, 2962 (s), 2913 (s), 1494 (m), 1452 (s), 1376 (m), 1032 (m), 703 (s); LRMS (ESI ) m/z = 293 $\left([\mathrm{M}+\mathrm{Na}]^{+}, 100 \%\right)$; HRMS $\left(\mathrm{ESI}^{+}\right)$calcd for $\mathrm{C}_{19} \mathrm{H}_{26} \mathrm{ONa}\left([\mathrm{M}+\mathrm{Na}]^{+}\right), 293.1881$, found 293.1884. 

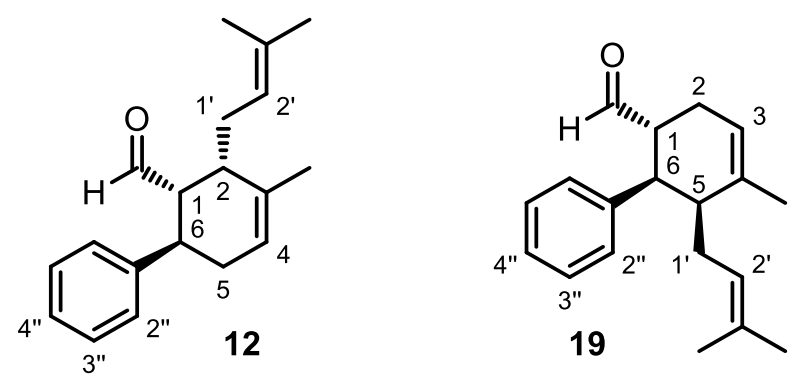

Cinnamaldehyde 18 (917 mg, $6.94 \mathrm{mmol}), E$-ocimene 8 (3.79 g, $27.8 \mathrm{mmol})$ and dichloromethane $(3 \mathrm{~mL})$ were added to a pressure vessel, sealed and pressurized to $19 \mathrm{kbar}$ for 3 days. The reactions was then diluted with dichloromethane $(20 \mathrm{~mL})$, evaporated on to silica and purified by column chromatography using diethyl ether : hexane (1:9) to afford as (3-methyl-2-(3-methylbut-2-en-1-yl)6-phenylcyclohex-3-en-1-yl)methanal 12 a clear colorless oil (144 mg, 8\%); $\mathbf{R}_{\mathbf{f}}$ (diethyl ether:hexane 1:4) 0.54; ${ }^{1} \mathrm{H}-\mathbf{N M R}\left(400 \mathrm{MHz}, \mathrm{CDCl}_{3}\right) \delta 9.46(1 \mathrm{H}, \mathrm{d}, J=3.6 \mathrm{~Hz}, \mathrm{CHO}), 7.28(2 \mathrm{H}, \mathrm{m}$, H2"), 7.21-7.16 (3H, m, H3"\& H4"), 5.49 (1H, br s, H4), 5.05 (1H, m, H2'), 3.35 (1H, ddd, J= 10.8, 10.8 \& $6.0 \mathrm{~Hz}, \mathrm{H} 6), 2.81(1 \mathrm{H}, \mathrm{ddd}, J=10.8,4.4 \& 3.6 \mathrm{~Hz}, \mathrm{H} 1), 2.52-2.40$ (2H, m, H2 \& H5a), $2.31\left(2 \mathrm{H}, \mathrm{t}, J=5.6 \mathrm{~Hz}, \mathrm{H}^{\prime}\right), 2.14(1 \mathrm{H}, \mathrm{m}, \mathrm{H} 5 \beta), 1.78\left(3 \mathrm{H}, \mathrm{s}, \mathrm{CH}_{3}\right), 1.66\left(3 \mathrm{H}, \mathrm{s}, \mathrm{CH}_{3}\right), 1.60(3 \mathrm{H}, \mathrm{s}$, $\left.\mathrm{CH}_{3}\right) ;{ }^{13} \mathbf{C}-\mathbf{N M R}\left(100 \mathrm{MHz}, \mathrm{CDCl}_{3}\right) \delta 205.80,144.0,136.3,132.3,128.7,127.7,126.6,123.7$, 121.6, 55.3, 42.2, 36.9, 34.0, 28.9, 25.7, 22.2, 18.0; IR (thin film) $v_{\max }=2964$ (m), 2914 (m), 1725 (s), 1604 (w), 1493 (m), 1447 (m), 1209 (w), 748 (m); LRMS (EI $) m / z=268\left(\mathrm{M}^{+\bullet}, 80 \%\right), 91$ $(100 \%)$; HRMS $\left(\mathrm{EI}^{+}\right)$calcd for $\mathrm{C}_{19} \mathrm{H}_{24} \mathrm{O}\left(\mathrm{M}^{+\bullet}\right)$ 268.1827, found 268.1824. A second fraction afforded (4-methyl-5-(3-methylbut-2-en-1-yl)-6-phenylcyclohex-3-en-1-yl)methanal 19 as a clear colorless oil (630 mg, 54\%); $\mathbf{R}_{\mathbf{f}}$ (diethyl ether:hexane 1:4) $0.48 ;{ }^{1} \mathbf{H}-\mathbf{N M R}$ (400 MHz, $\mathrm{CDCl}_{3}$ ) $\delta 9.42$ (1H, d, $J=3.6 \mathrm{~Hz}, \mathrm{CHO}), 7.27$ (2H, m, H3"), 7.20-7.16 (3H, m, H2"\& H4"), 5.50 (1H, m, H3), $4.85(1 \mathrm{H}, \mathrm{m}, \mathrm{H} 2$ '), 3.35 (1H, dd, $J=10.4 \& 5.2 \mathrm{~Hz}, \mathrm{H} 6), 2.99(1 \mathrm{H}, \mathrm{dddd}, J=10.4,7.2,7.2$ \& $3.2 \mathrm{~Hz}$, H1), 2.28-2.22 (3H, m, H2 \& H5), 1.97 (2H, dd, $J=11.6 \& 6.4, \mathrm{H} 1$ '), $1.75\left(3 \mathrm{H}, \mathrm{s}, \mathrm{CH}_{3}\right), 1.57$ (3H, $\left.\mathrm{s}, \mathrm{CH}_{3}\right), 1.27\left(3 \mathrm{H}, \mathrm{s}, \mathrm{CH}_{3}\right) ;{ }^{13} \mathbf{C}-\mathbf{N M R}\left(100 \mathrm{MHz} \mathrm{CDCl}_{3}\right) \delta$ 204.1, 141.1, 137.9, 131.42, 128.8, $128.2,126.4,122.9,119.2,46.5,44.0,43.5,27.3,25.7,24.5,22.7,17.5$; IR (thin film) $v_{\max }=2964$ (m), 2913 (m), 1724 (s), 1602 (w), 1495 (m), 1452 (m), 1377 (m), 750 (m); LRMS (EI $\left.{ }^{+}\right) m / z=268$ $\left(\mathrm{M}^{+\bullet}, 80 \%\right), 91(100 \%)$; HRMS $\left(\mathrm{EI}^{+}\right)$calcd for $\mathrm{C}_{19} \mathrm{H}_{24} \mathrm{O}\left(\mathrm{M}^{+\bullet}\right) 268.1827$, found 268.1824.

To a solution of (3-methyl-2-(3-methylbut-2-en-1-yl)-6-phenylcyclohex-3-en-1-yl)methanol 10 $(171 \mathrm{mg}, 633 \mu \mathrm{mol})$ in dichloromethane $(20 \mathrm{~mL})$ was added Dess-Martin periodinane (403 mg, 950 
$\mu \mathrm{mol})$ and the mixture was stirred at room temperature for $1 \mathrm{~h}$. The reaction was then evaporated on to silica under reduced pressure and purified by column chromatography using diethyl ether : hexane (1:9) to afford 3-methyl-2-(3-methylbut-2-en-1-yl)-6-phenylcyclohex-3-en-1-yl)methanal 12 as a clear colorless oil (132 $\mathrm{mg}, 78 \%)$.

\section{General procedure for high pressure reactions (Table 1)}

Dienophile (917 mg, $6.94 \mathrm{mmol}), E$-ocimene 8 (3.79 g, $27.8 \mathrm{mmol})$ and dichloromethane $(3 \mathrm{~mL})$ were added to a pressure vessel, sealed and pressurized to 19 kbar for 3 days. The reactions were then diluted with dichloromethane $(20 \mathrm{~mL})$, evaporated onto silica and purified by column chromatography to afford the corresponding Diels-Alder adducts.

Reduction to alcohols. To a solution of Diels-Alder adducts $(251 \mathrm{mg}, 0.841 \mathrm{mmol})$ in tetrahydrofuran $(20 \mathrm{~mL})$ was added lithium aluminum hydride in tetrahydrofuran $(3.37 \mathrm{~mL}, 1 \mathrm{M}$, $3.37 \mathrm{mmol}$ ) dropwise over $5 \mathrm{~min}$. After $1 \mathrm{~h}$ of stirring at room temperature the reaction was cooled to $0^{\circ} \mathrm{C}$ and ethyl acetate $(10 \mathrm{~mL})$ was added dropwise over 10 minutes. The reaction was then diluted with diethyl ether $(80 \mathrm{~mL})$ and a saturated solution of potassium sodium tartrate $(80 \mathrm{~mL})$ and stirred vigorously for $30 \mathrm{~min}$. The diethyl ether layer was then separated and the aqueous layer re-extracted with diethyl ether $(2 \times 50 \mathrm{~mL})$. The combined organic layers were washed with brine $(50 \mathrm{~mL})$ and evaporated on to silica with purification by column chromatography to afford the corresponding alcohols.

Reduction to aldehydes. To a solution of Diels-Alder adducts (454 mg, $1.71 \mathrm{mmol}$ ) in dichloromethane $(20 \mathrm{~mL})$ was added diisobutylaluminum hydride in dichloromethane $(5.13 \mathrm{~mL}, 1$ $\mathrm{M}, 5.13 \mathrm{mmol}$ ) at $-78^{\circ} \mathrm{C}$. After $30 \mathrm{~min}$, the reaction was quenched with saturated aqueous sodium potassium tartrate $(50 \mathrm{~mL})$, and stirred vigorously for $30 \mathrm{~min}$. The dichloromethane layer was then separated and the aqueous layer re-extracted with dichloromethane $(2 \times 50 \mathrm{~mL})$. The combined organic layers were washed with brine $(50 \mathrm{~mL})$ and evaporated on to silica with purification by column chromatography to afford the corresponding aldehydes.

\section{2-Bromo-5-methoxybenzene-1,3-diol (23) ${ }^{[4]}$}<smiles>COc1cc(O)c(Br)c(O)c1</smiles> 
To a solution of 5-methoxyresorcinol (1.92 g, $13.7 \mathrm{mmol})$ in chloroform $(100 \mathrm{~mL})$ and dioxane $(5$ $\mathrm{mL}$ ) at $0^{\circ} \mathrm{C}$ was added portion-wise over $1 \mathrm{~h}$ a solution of 2,4,4,6-tetrabromo-2,5-cyclohexadienone $(6.79 \mathrm{~g}, 16.6 \mathrm{mmol})$ in chloroform $(20 \mathrm{~mL})$. The resulting reaction mixture was stirred at $0^{\circ} \mathrm{C}$ for $5 \mathrm{~h}$ and then concentrated under reduced pressure. Purification via silica gel chromatography using dichloromethane followed by recrystallization from dichloromethane gave 2-bromo-5methoxybenzene-1,3-diol 23 as white crystals (1.60 g, 54\%); $\mathbf{R}_{\mathbf{f}}(\mathrm{DCM}) 0.13$; m.p. $73-75^{\circ} \mathrm{C}\left(\right.$ (lit. $^{[5]}$ m.p. 73-75 $\left.{ }^{\circ} \mathrm{C}\right) ;{ }^{1} \mathbf{H}-\mathbf{N M R}\left(400 \mathrm{MHz}, \mathrm{CDCl}_{3}\right) \delta 6.22(2 \mathrm{H}, \mathrm{s}, \mathrm{CH}), 5.41(2 \mathrm{H}, \mathrm{s}, \mathrm{OH}), 3.75(3 \mathrm{H}, \mathrm{s}$, $\left.\mathrm{CH}_{3} \mathrm{O}\right) ;{ }^{13} \mathbf{C}-\mathbf{N M R}\left(100 \mathrm{MHz}, \mathrm{CDCl}_{3}\right) \delta 160.7,153.3,94.7,90.6,55.5$; IR (thin film) $v_{\max }=3453$ (m), 2940 (w), 2849 (w), 1597 (vs), 1506 (m), 1444 (m), 1368 (m), 1323 (w) cm ${ }^{-1}$; LRMS (ESI ${ }^{-}$) $m / z=217\left(\mathrm{C}_{7} \mathrm{H}_{6} \mathrm{O}_{3}{ }^{79} \mathrm{Br},[\mathrm{M}-\mathrm{H}]^{-}, 22 \%\right), 219\left(\mathrm{C}_{7} \mathrm{H}_{6} \mathrm{O}_{3}{ }^{81} \mathrm{Br},[\mathrm{M}-\mathrm{H}]^{-}, 21 \%\right), 122(100 \%)$; HRMS (ESI ${ }^{-}$) calcd for $\mathrm{C}_{7} \mathrm{H}_{6} \mathrm{O}_{3}{ }^{79} \mathrm{Br}\left([\mathrm{M}-\mathrm{H}]^{-}\right)$216.9500, found 216.9500 , calcd for $\mathrm{C}_{7} \mathrm{H}_{6} \mathrm{O}_{3}{ }^{81} \mathrm{Br}\left([\mathrm{M}-\mathrm{H}]^{-}\right)$ 218.9480, found 218.9482 .

\section{2-Bromo-5-methoxy-1,3-bis(methoxymethoxy)benzene $(13)^{[6]}$}

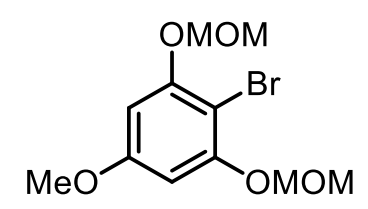

Sodium hydride 60\% dispersion in mineral oil $(557 \mathrm{mg}, 13.9 \mathrm{mmol})$ and hexanes $(60 \mathrm{~mL})$ were added to a flask and stirred vigorously at room temperature for $30 \mathrm{~min}$. The mixture was then allowed to settle and the majority of the hexane removed by pipette. Dimethylformamide $(30 \mathrm{~mL})$ was added to the flask followed by cooling to $0^{\circ} \mathrm{C}$. 2-Bromo-5-methoxybenzene-1,3-diol 23 (1.02 g, $4.66 \mathrm{mmol})$ was then added portion wise over $20 \mathrm{~min}$ followed by methoxymethyl chloride $(881 \mu \mathrm{L}$, $11.6 \mathrm{mmol}$ ) dropwise over $10 \mathrm{~min}$. After warming to room temperature the resulting solution was stirred for $16 \mathrm{~h}$ and then diluted with water $(60 \mathrm{~mL})$. Extraction with ether $(3 \times 50 \mathrm{~mL})$, drying over magnesium sulfate, concentration under reduced pressure and purification by silica gel chromatography using diethyl ether : hexane (1:4) gave 2-bromo-5-methoxy-1,3bis(methoxymethoxy)benzene 13 as a clear colorless oil (946 $\mathrm{mg}, 66 \%)$; $\mathbf{R}_{\mathbf{f}}$ (diethyl ether:hexane 3:7) 0.26; ${ }^{1} \mathbf{H}-\mathbf{N M R}\left(400 \mathrm{MHz}, \mathrm{CDCl}_{3}\right) \delta 6.46(2 \mathrm{H}, \mathrm{s}, \mathrm{CH}), 5.22\left(4 \mathrm{H}, \mathrm{s}, \mathrm{CH}_{3} \mathrm{OCH}_{2} \mathrm{O}\right), 3.77(3 \mathrm{H}, \mathrm{s}$, $\left.\mathrm{CH}_{3} \mathrm{O}\right), 3.51\left(6 \mathrm{H}, \mathrm{s}, \mathrm{CH}_{3} \mathrm{OCH}_{2} \mathrm{O}\right) ;{ }^{13} \mathbf{C}-\mathbf{N M R}\left(100 \mathrm{MHz}, \mathrm{CDCl}_{3}\right) \delta$ 160.0, 155.1, 96.2, 95.0, 94.6, 56.2, 55.4; IR (thin film) $v_{\max }=2963$ (s), 2911 (s), 2831 (m), 1605 (s), 1537 (w), 1482 (s), 1391 (m), $1303(\mathrm{~m}) \mathrm{cm}^{-1}$; LRMS $\left(\mathrm{EI}^{+}\right) \mathrm{m} / z=306\left(\mathrm{C}_{11} \mathrm{H}_{15} \mathrm{O}_{5}{ }^{79} \mathrm{Br}, \mathrm{M}^{+\bullet}, 100 \%\right), 308\left(\mathrm{C}_{11} \mathrm{H}_{15} \mathrm{O}_{5}{ }^{81} \mathrm{Br}, \mathrm{M}^{+\bullet}\right.$, 98\%); HRMS (EI $\left.{ }^{+}\right)$calcd for $\mathrm{C}_{11} \mathrm{H}_{15} \mathrm{O}_{5}{ }^{79} \mathrm{Br}\left(\mathrm{M}^{+\bullet}\right)$ 306.0103, found 306.0103, calcd for $\mathrm{C}_{11} \mathrm{H}_{15} \mathrm{O}_{5}{ }^{81} \mathrm{Br}\left(\mathrm{M}^{+\bullet}\right)$ 308.0082, found 308.0066. 


\section{1,3,5-Tris(methoxymethoxy)benzene $(24)^{[7]}$}

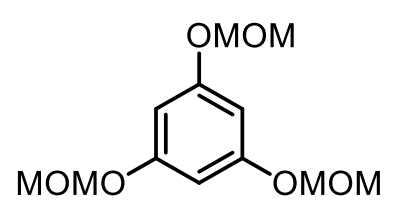

Sodium hydride $60 \%$ dispersion in mineral oil ( $5.70 \mathrm{~g}, 143 \mathrm{mmol})$ and hexane (40 $\mathrm{mL})$ were added to a flask and stirred vigorously at room temperature for $30 \mathrm{~min}$. The mixture was then allowed to settle and the majority of the hexane removed by pipette. Dimethylformamide $(80 \mathrm{~mL})$ was added to the flask followed by cooling to $0^{\circ} \mathrm{C}$. Phloroglucinol $(4.50 \mathrm{~g}, 35.7 \mathrm{mmol})$ was then added portion wise over $10 \mathrm{~min}$ followed by methoxymethyl chloride $(8.97 \mathrm{~mL}, 118 \mathrm{mmol})$ dropwise over $10 \mathrm{~min}$. After warming to room temperature the resulting solution was stirred for $16 \mathrm{~h}$ and then diluted with water $(100 \mathrm{~mL})$. Extraction with ether $(3 \times 50 \mathrm{~mL})$, drying over magnesium sulfate, concentration under reduced pressure and purification by silica gel chromatography using ethyl acetate : hexane (1:4) afforded 1,3,5-tris(methoxymethoxy)benzene 24 as a clear colorless oil (5.68 g, 62\%); $\mathbf{R}_{\mathbf{f}}$ (ethyl acetate:hexane 1:4) $0.22 ;{ }^{1} \mathrm{H}-\mathbf{N M R}\left(400 \mathrm{MHz}, \mathrm{CDCl}_{3}\right) \delta 6.41(3 \mathrm{H}, \mathrm{s}, \mathrm{CH}), 5.13(6 \mathrm{H}, \mathrm{s}$, $\left.\mathrm{CH}_{3} \mathrm{OCH}_{2} \mathrm{O}\right), 3.47\left(9 \mathrm{H}, \mathrm{s}, \underline{\mathrm{CH}}_{3} \mathrm{OCH}_{2} \mathrm{O}\right) ;{ }^{13} \mathbf{C}-\mathbf{N M R}\left(100 \mathrm{MHz}, \mathrm{CDCl}_{3}\right) \delta 158.9,98.4,94.5,56.1$; IR (thin film) $v_{\max }=2955(\mathrm{w}), 2901(\mathrm{~m}), 2827(\mathrm{~m}), 1598(\mathrm{~s}), 1471(\mathrm{~m}), 1439(\mathrm{w}), 1399(\mathrm{~m}), 1314(\mathrm{w})$ $\mathrm{cm}^{-1}$; LRMS $\left(\mathrm{EI}^{+}\right) \mathrm{m} / z=258\left(\mathrm{M}^{+\bullet}, 55 \%\right), 45\left(\mathrm{CH}_{3} \mathrm{OCH}_{2}{ }^{+}, 100 \%\right)$; HRMS $\left(\mathrm{EI}^{+}\right)$calcd for $\mathrm{C}_{12} \mathrm{H}_{18} \mathrm{O}_{6}$ $\left(\mathrm{M}^{+\bullet}\right)$ 258.1103, found 258.1116.

\section{2-Bromo-1,3,5-tris(methoxymethoxy)benzene $(15)^{[7]}$}<smiles>COc1cc(OC)c(Br)c(OC)c1</smiles>

To a solution of 1,3,5-tris(methoxymethoxy)benzene 24 (5.68 g, $22.0 \mathrm{mmol})$ in dichloromethane $(60 \mathrm{~mL})$ was added $N$-bromosuccinimide $(4.11 \mathrm{~g}, 23.1 \mathrm{mmol})$ followed by stirring at room temperature for $1 \mathrm{~h}$. The reaction was then evaporated on to silica under reduced pressure and purified by silica gel chromatography using ethyl acetate : hexane (3:17) to give 2-bromo-1,3,5tris(methoxymethoxy)benzene 15 as a white solid (7.34 g, 98\%); $\mathbf{R}_{\mathbf{f}}$ (ethyl acetate:hexane 1:4) 0.20; m.p. $35-40^{\circ} \mathrm{C} ;{ }^{1} \mathrm{H}-\mathrm{NMR}\left(400 \mathrm{MHz}, \mathrm{CDCl}_{3}\right) \delta 6.60(2 \mathrm{H}, \mathrm{s}, \mathrm{CH}), 5.23\left(4 \mathrm{H}, \mathrm{s}, \mathrm{CH}_{3} \mathrm{OCH}_{2} \mathrm{O}\right), 5.14$ $\left(2 \mathrm{H}, \mathrm{s}, \mathrm{CH}_{3} \mathrm{OCH}_{2} \mathrm{O}\right), 3.52\left(6 \mathrm{H}, \mathrm{s}, \underline{\mathrm{CH}}_{3} \mathrm{OCH}_{2} \mathrm{O}\right), 3.47\left(3 \mathrm{H}, \mathrm{s}, \underline{\mathrm{CH}}_{3} \mathrm{OCH}_{2} \mathrm{O}\right) ;{ }^{13} \mathbf{C}-\mathbf{N M R}(100 \mathrm{MHz}$, $\left.\mathrm{CDCl}_{3}\right) \delta 157.7,155.2,98.5,96.1,95.1,94.6,56.4,56.1$; IR (thin film) $v_{\max }=2957(\mathrm{~m}), 2907(\mathrm{~m})$, 2828 (m), 1588 (vs), 1469 (m), 1392 (s), 1307 (w), 1232 (m) cm ${ }^{-1}$; LRMS (EI $\left.{ }^{+}\right) \mathrm{m} / z=336$ $\left(\mathrm{C}_{12} \mathrm{H}_{17} \mathrm{O}_{6}{ }^{79} \mathrm{Br}, \mathrm{M}^{+\bullet}, 100 \%\right), 338\left(\mathrm{C}_{12} \mathrm{H}_{17} \mathrm{O}_{6}{ }^{81} \mathrm{Br}, \mathrm{M}^{+\bullet}, 100 \%\right)$; HRMS (EI $\left.{ }^{+}\right)$calcd for $\mathrm{C}_{12} \mathrm{H}_{17}{ }^{79} \mathrm{BrO}_{6}$ $\left(\mathrm{M}^{+\bullet}\right)$ 336.0208, found 336.0208, calcd for $\mathrm{C}_{12} \mathrm{H}_{17}{ }^{81} \mathrm{BrO}_{6}\left(\mathrm{M}^{+\bullet}\right)$ 338.0188, found 338.0192. 


\section{2,6-bis(methoxymethoxy) phenyl) methanone (14)}

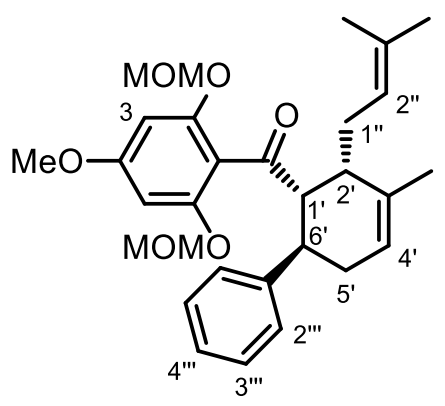

2-Bromo-5-methoxy-1,3-bis(methoxymethoxy)benzene 13 (230 mg, $748 \mu \mathrm{mol}$ ) and tetrahydrofuran $(15 \mathrm{~mL})$ were added to a flask and cooled to $-78^{\circ} \mathrm{C}$ under a nitrogen atmosphere. Butyl lithium (575 $\mu \mathrm{L}, 1.6 \mathrm{M}, 748 \mu \mathrm{mol}$ ) was then added dropwise over $5 \mathrm{~min}$ and the resulting solution stirred for 30 min. A solution of (3-methyl-2-(3-methylbut-2-en-1-yl)-6-phenylcyclohex-3-en-1-yl)methanal 12 (66.9 $\mathrm{mg}, 249 \mu \mathrm{mol})$ in tetrahydrofuran $(5 \mathrm{~mL})$ was then added dropwise over $5 \mathrm{~min}$ followed by stirring at $-78^{\circ} \mathrm{C}$ for $15 \mathrm{~min}$. After this the reaction was allowed to warm to room temperature and stirred for a further 30 minutes followed by quenching with a saturated solution of ammonium chloride $(30 \mathrm{~mL})$. Extraction with diethyl ether $(3 \times 30 \mathrm{~mL})$, drying over magnesium sulfate and purification by column chromatography using diethyl ether : hexane (3:7) gave a mixture of the alcohol diastereomer products; $\mathbf{R}_{\mathbf{f}}$ (diethyl ether:hexane 1:1) $0.11 \& 0.22$; The alcohol products, Dess-Martin periodinane $(211 \mathrm{mg}, 498 \mu \mathrm{mol})$ and dichloromethane $(15 \mathrm{~mL})$ were added to a flask and stirred at room temperature for $1 \mathrm{~h}$. The reaction was then evaporated on to silica under reduced pressure and purified by column chromatography using diethyl ether : hexane (3:7) to afford (3methyl-2-(3-methylbut-2-en-1-yl)-6-phenylcyclohex-3-en-1-yl) (4-methoxy-2,6-bis(methoxymethoxy)phenyl)methanone $\mathbf{1 4}$ as colorless oil (87.5 mg, 71\% over 2 steps); $\mathbf{R}_{\mathbf{f}}$ (diethyl ether:hexane 1:1) 0.19; ${ }^{1} \mathbf{H}-\mathbf{N M R}\left(400 \mathrm{MHz} \mathrm{CDCl}_{3}\right) \delta 7.31$ (2H, d, $\left.J=8.0 \mathrm{~Hz}, \mathrm{H} 2 " '\right), 7.23$ (2H, t, $J$ $\left.=8.0 \mathrm{~Hz}, \mathrm{H} 3{ }^{\prime \prime}\right), 7.10\left(1 \mathrm{H}, \mathrm{t}, J=7.2 \mathrm{~Hz}, \mathrm{H} 4{ }^{\prime \prime}\right), 6.36(2 \mathrm{H}, \mathrm{s}, \mathrm{H} 3), 5.40(1 \mathrm{H}, \mathrm{br} \mathrm{s}, \mathrm{H} 4 '), 5.11(1 \mathrm{H}, \mathrm{m}$, H2"), $5.01\left(4 \mathrm{H}, \mathrm{s}, \underline{\mathrm{CH}}_{2} \mathrm{OCH}_{3}\right), 3.84\left(1 \mathrm{H}, \mathrm{dd}, J=10.8 \& 3.2 \mathrm{~Hz}, \mathrm{H} 1^{\prime}\right), 3.77\left(3 \mathrm{H}, \mathrm{s}, \mathrm{OCH}_{3}\right), 3.48(1 \mathrm{H}$, m, H6'), 3.38 (6H, s, $\left.\mathrm{CH}_{2} \mathrm{OCH}_{3}\right), 2.51$ (1H, m, H5' $\beta$ ), 2.36-2.34 (3H, m, H2' \& H1"), 2.17 (1H, m, $\left.5^{\prime} \alpha\right), 1.72\left(3 \mathrm{H}, \mathrm{s}, \mathrm{CH}_{3}\right), 1.67\left(3 \mathrm{H}, \mathrm{s}, \mathrm{CH}_{3}\right), 1.61\left(3 \mathrm{H}, \mathrm{s}, \mathrm{CH}_{3}\right) ;{ }^{13} \mathbf{C}-\mathbf{N M R}\left(100 \mathrm{MHz}, \mathrm{CDCl}_{3}\right) \delta 203.3$, $161.8,156.5,146.8,138.3,130.9,128.2,127.7,125.5,124.6,120.6,114.8,94.9,94.5,58.2,56.1$, 55.4, 40.7, 36.8, 34.5, 29.6, 25.8, 23.6, 18.0; IR (thin film) $v_{\max }=2960$ (m), 2912 (m), 1695 (m), 1605 (vs), 1452 (m), 1391 (w), 1215 (m), 1151 (vs); LRMS (EI $\left.{ }^{+}\right) m / z=494\left(\mathrm{M}^{+\bullet}, 2 \%\right), 197$ (100\%); HRMS $\left(\mathrm{EI}^{+}\right)$calcd for $\mathrm{C}_{30} \mathrm{H}_{38} \mathrm{O}_{6}\left(\mathrm{M}^{+\bullet}\right)$ 494.2668, found 494.2669. 
( \pm -Panduratin A $\left(1^{[8]}\right.$

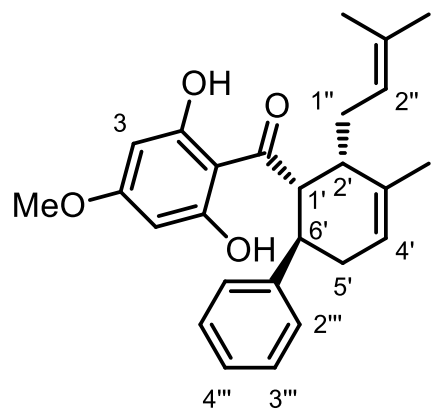

(3-Methyl-2-(3-methylbut-2-en-1-yl)-6-phenylcyclohex-3-en-1-yl) (4-methoxy-2,6-bis(methoxymethoxy)phenyl)methanone $14(83.4 \mathrm{mg}, 169 \mu \mathrm{mol})$, methanol $(10 \mathrm{~mL})$ and 2 drops of concentrated hydrochloric acid were added to a flask and heated at $50^{\circ} \mathrm{C}$ with stirring for $2 \mathrm{~h}$. The reaction mixture was then neutralized with saturated sodium bicarbonate solution, extracted with ethyl acetate $(2 \times 30 \mathrm{~mL})$ and dried over magnesium sulfate. Filtration, concentration under reduced pressure and purification via silica gel chromatography using diethyl ether : hexane (3:7) afforded ( \pm )-panduratin A 1 as a yellow solid (48.3 mg, 70\%); $\mathbf{R}_{\mathbf{f}}$ (diethyl ether:hexane 1:1) 0.35; m.p. 154$156^{\circ} \mathrm{C}$ (lit. ${ }^{[8]}$ m.p. $\left.157-157.5^{\circ} \mathrm{C}\right) ;{ }^{1} \mathbf{H}-\mathbf{N M R}\left(400 \mathrm{MHz}, \mathrm{CD}_{3} \mathrm{OD}\right) \delta 7.18-7.16$ (4H, m, H2"' \& H3"'), 7.05 (1H, m, H4'"), 5.88 (2H, s, H3), 5.41 (1H br s, H4'), 4.88 (1H, obscured by HOD, H2"), 4.77 (1H, dd, $\left.J=11.6 \& 4.4, \mathrm{H}^{\prime}\right), 3.74$ (3H, s, OMe), 3.38 (1H, ddd, $J=10.8,10.8,6.4$ Hz, H6'), 2.65

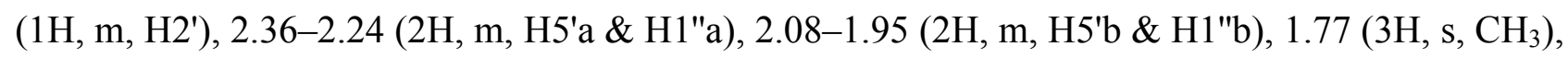
$1.50\left(6 \mathrm{H}, \mathrm{s}, \mathrm{CH}_{3}\right) ;{ }^{13} \mathbf{C}-\mathbf{N M R}\left(100 \mathrm{MHz}, \mathrm{CD}_{3} \mathrm{OD}\right) \delta 208.1,167.0,165.4,148.5,138.4,132.4,129.2$, $128.3,126.4,125.7,122.0,107.2,94.4,55.76,55.0,43.4,38.4,37.1,29.8,25.9,23.0,18.1$; IR (thin film) $v_{\max }=3401(\mathrm{~s}), 2964(\mathrm{w}), 2914$ (w), 1627 (s), 1581 (m), 1424 (w), 1231 (m), $1162(\mathrm{~m}) \mathrm{cm}^{-1}$; LRMS $\left(\mathrm{ESI}^{-}\right) \mathrm{m} / z=405\left([\mathrm{M}-\mathrm{H}]^{-}, 100 \%\right)$; HRMS $\left(\mathrm{ESI}^{-}\right)$calcd for $\mathrm{C}_{26} \mathrm{H}_{29} \mathrm{O}_{4}\left([\mathrm{M}-\mathrm{H}]^{-}\right) 405.2066$, found 405.2066 .

( \pm )-((1R,2S,6R)-3-Methyl-2-(3-methylbut-2-en-1-yl)-6-phenylcyclohex-3-en-1-yl)

$(2,4,6-$ tris(methoxymethoxy)phenyl)methanone (16)

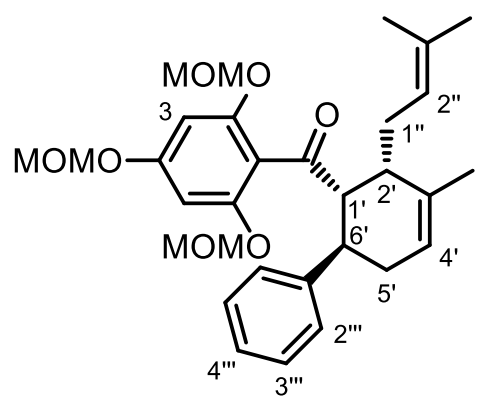

2-Bromo-1,3,5-tris(methoxymethoxy)benzene 15 (277 mg, $822 \mu \mathrm{mol})$ and tetrahydrofuran (15 mL) were added to a flask and cooled to $-78^{\circ} \mathrm{C}$ under a nitrogen atmosphere. Butyl lithium $(514 \mu \mathrm{L}$, $1.6 \mathrm{M}, 823 \mu \mathrm{mol}$ ) was then added dropwise over $5 \mathrm{~min}$ and the resulting solution stirred for $30 \mathrm{~min}$. 
A solution of (3-methyl-2-(3-methylbut-2-en-1-yl)-6-phenylcyclohex-3-en-1-yl)methanal 12 (55.2 $\mathrm{mg}, 206 \mu \mathrm{mol})$ in tetrahydrofuran $(5 \mathrm{~mL})$ was then added dropwise over 5 min followed by stirring at $-78^{\circ} \mathrm{C}$ for $15 \mathrm{~min}$. After this the reaction was allowed to warm to room temperature and stirred for a further 30 min followed by quenching with a saturated solution of ammonium chloride (30 $\mathrm{mL})$. Extraction with diethyl ether $(3 \times 30 \mathrm{~mL})$, drying over magnesium sulfate and purification by column chromatography using diethyl ether : hexane (3:7) )gave a mixture of the alcohol diastereomer products; $\mathbf{R}_{\mathbf{f}}$ (diethyl ether:hexane 1:1) $0.20 \& 0.28$; The alcohol products, DessMartin periodinane $(131 \mathrm{mg}, 309 \mu \mathrm{mol})$ and dichloromethane $(15 \mathrm{~mL})$ were added to a flask and stirred at room temperature for $1 \mathrm{~h}$. The reaction was then evaporated on to silica under reduced pressure and purified by column chromatography using diethyl ether : hexane (3:7) to afford (3methyl-2-(3-methylbut-2-en-1-yl)-6-phenylcyclohex-3-en-1-yl) (2,4,6-tris(methoxymethoxy)phenyl) methanone 16 as colorless oil (78.9 mg, 73\% over 2 steps); $\mathbf{R}_{\mathbf{f}}$ (diethyl ether:hexane 1:1) 0.37; ${ }^{1} \mathbf{H}-$ NMR (400 MHz, $\left.\mathrm{CDCl}_{3}\right) \delta 7.31\left(2 \mathrm{H}, \mathrm{d}, J=7.6 \mathrm{~Hz}, \mathrm{H} 2{ }^{\prime \prime}\right), 7.23\left(2 \mathrm{H}, \mathrm{t}, J=7.2 \mathrm{~Hz}, \mathrm{H} 3{ }^{\prime \prime}\right), 7.11(1 \mathrm{H}, \mathrm{t}$, $\left.J=7.2 \mathrm{~Hz}, \mathrm{H} 4{ }^{\prime \prime}\right), 6.48(2 \mathrm{H}, \mathrm{s}, \mathrm{H} 3), 5.41$ (1H, br s, H4'), $5.13\left(3 \mathrm{H}, \mathrm{s}, \mathrm{H} 2 "\right.$ \& $\left.\underline{\mathrm{CH}}_{2} \mathrm{OCH}_{3}\right), 5.00(4 \mathrm{H}, \mathrm{s}$, $\left.\underline{\mathrm{CH}}_{2} \mathrm{OCH}_{3}\right), 3.81\left(1 \mathrm{H}, \mathrm{dd}, J=11.2 \& 3.2 \mathrm{~Hz}, \mathrm{H} 1^{\prime}\right), 3.52-3.47\left(4 \mathrm{H}, \mathrm{m}, \mathrm{H} 6{ }^{\prime} \& \mathrm{CH}_{2} \mathrm{OCH}_{3}\right), 3.37(6 \mathrm{H}$, s, $\left.\mathrm{CH}_{2} \mathrm{OCH}_{3}\right), 2.51\left(1 \mathrm{H}, \mathrm{m}, \mathrm{H} 5^{\prime} \beta\right), 2.36-2.33$ (3H, m, H2' \& H1"), 2.17 (1H, m, H5' $\left.\alpha\right), 1.73$ (3H, s, $\left.\mathrm{CH}_{3}\right), 1.67\left(3 \mathrm{H}, \mathrm{s}, \mathrm{CH}_{3}\right), 1.61\left(3 \mathrm{H}, \mathrm{s}, \mathrm{CH}_{3}\right) ;{ }^{13} \mathbf{C}-\mathbf{N M R}\left(100 \mathrm{MHz}, \mathrm{CDCl}_{3}\right) \delta 203.3,159.4,156.3$, 146.7, 138.3, 130.9, 128.3, 127.7, 125.5, 124.6, 120.7, 115.9, 97.0, 94.5, 94.4, 58.3, 56.3, 56.2, 40.6, 36.7, 34.4, 29.6, 25.8, 23.6, 18.0; IR (thin film) $v_{\max }=2959$ (m), 2911 (m), 1697 (m), 1605 (s), 1451 (w), 1217 (w), 1155 (s), 1050 (vs); LRMS (EI $\left.{ }^{+}\right) m / z=524\left(\mathbf{M}^{+\bullet}, 3 \%\right), 389$ (100\%); HRMS $\left(\mathrm{EI}^{+}\right)$calcd for $\mathrm{C}_{31} \mathrm{H}_{40} \mathrm{O}_{7}\left(\mathrm{M}^{+\bullet}\right)$ 524.2774, found 524.2794.

\section{$( \pm)-4-H y d r o x y p a n d u r a t i n ~ A ~(2)^{[9]}$}

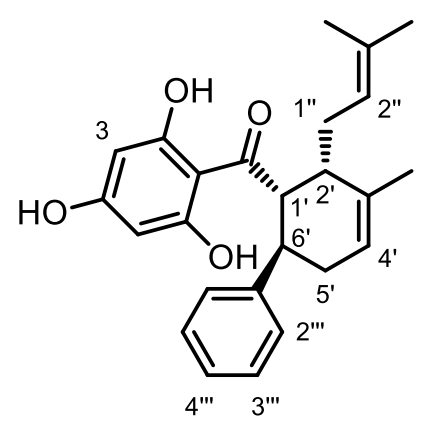

(3-Methyl-2-(3-methylbut-2-en-1-yl)-6-phenylcyclohex-3-en-1yl)

(2,4,6-tris(methoxymethoxy)phenyl) methanone $16(69.7 \mathrm{mg}, 141 \mu \mathrm{mol})$, methanol $(10 \mathrm{~mL})$ and 2 drops of concentrated hydrochloric acid were added to a flask and heated at $50^{\circ} \mathrm{C}$ with stirring for $2 \mathrm{~h}$. The reaction mixture was then neutralized with saturated sodium bicarbonate solution, extracted with ethyl acetate $(2 \times 30 \mathrm{~mL})$ and dried over magnesium sulfate. Filtration, concentration under reduced pressure and purification via silica gel chromatography using diethyl ether : hexane (2:3) afforded 
( \pm )-4-hydroxypanduratin A 2 as a pale yellow oil (39.3 $\mathrm{mg}, 71 \%$ ); $\mathbf{R}_{\mathbf{f}}$ (diethyl ether:hexane 1:1)

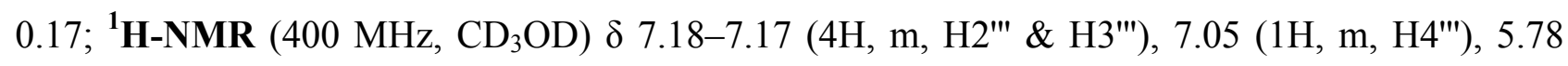
(2H, s, H3), 5.40 (1H, br s, H4'), 4.91 (1H, obscured by HOD, H2"), 4.76 (1H, dd, J= 12.0, 4.8 Hz, H1'), 3.37 (1H, ddd, $J=10.4,10.4,6.4, \mathrm{H6}$ '), 2.65 (1H, m, H2'), 2.36-2.22 (2H, m, H5'a \& H1"a), 2.08-1.94 (2H, m, H5'b \& H1"b), $1.77\left(3 \mathrm{H}, \mathrm{s}, \mathrm{CH}_{3}\right), 1.51\left(6 \mathrm{H}, \mathrm{s}, \mathrm{CH}_{3}\right) ;{ }^{13} \mathrm{C}-\mathbf{N M R}(100 \mathrm{MHz}$, $\left.\mathrm{CD}_{3} \mathrm{OD}\right) \delta 207.8,165.5,148.6,138.4,132.2,129.1,128.2,126.4,125.7,121.9,106.5,95.8(2 \times \mathrm{C})$, 54.8, 43.8, 38.4, 37.1, 29.9, 25.9, 23.0, 18.0; IR (thin film) $v_{\max }=3400(v s), 2958(w), 2913(w)$, 1623 (s), 1597 (m), 1492 (w), 1436 (w), 1216 (w) cm ${ }^{-1}$; LRMS (ESI $\left.{ }^{-}\right) m / z=391\left([\mathrm{M}-\mathrm{H}]^{-}, 100 \%\right)$;

HRMS $\left(\mathrm{ESI}^{-}\right)$calcd for $\mathrm{C}_{25} \mathrm{H}_{27} \mathrm{O}_{4}\left([\mathrm{M}-\mathrm{H}]^{-}\right)$391.1909, found 391.1909.

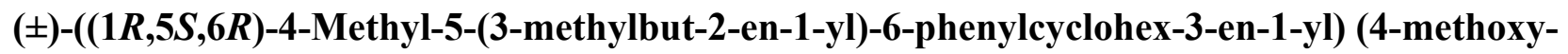

\section{2,6-bis(methoxymethoxy)phenyl) methanone (20)}

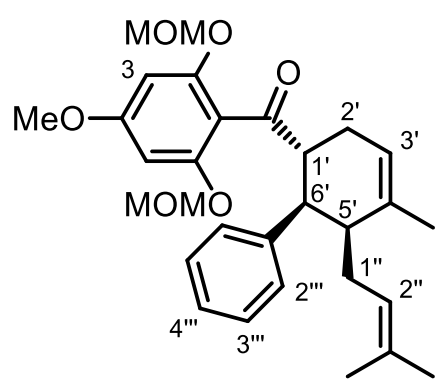

2-Bromo-5-methoxy-1,3-bis(methoxymethoxy)benzene $\quad \mathbf{1 3} \quad(229.7 \quad \mathrm{mg}, \quad 748 \quad \mu \mathrm{mol})$ and tetrahydrofuran $(20 \mathrm{~mL})$ were added to a flask and cooled to $-78^{\circ} \mathrm{C}$ under a nitrogen atmosphere. Butyl lithium $(575 \mu \mathrm{L}, 1.3 \mathrm{M}, 748 \mu \mathrm{mol})$ was then added dropwise over $5 \mathrm{~min}$ and the resulting solution stirred for $30 \mathrm{~min}$. A solution of (3-methyl-2-(3-methylbut-2-en-1-yl)-6-phenylcyclohex-3en-1-yl)methanal $19(66.9 \mathrm{mg}, 249 \mu \mathrm{mol})$ in tetrahydrofuran $(5 \mathrm{~mL})$ was then added dropwise over 5 min followed by stirring at $-78^{\circ} \mathrm{C}$ for $15 \mathrm{~min}$. After this the reaction was allowed to warm to room temperature and stirred for a further 30 min followed by quenching with a saturated solution of ammonium chloride $(30 \mathrm{~mL})$. Extraction with diethyl ether $(3 \times 20 \mathrm{~mL})$, drying over magnesium sulfate and purification by column chromatography using diethyl ether : hexane (1:3) gave a mixture of the alcohol diastereomer products; $\mathbf{R}_{\mathbf{f}}$ (diethyl ether:hexane 1:1) $0.11 \& 0.19$; The alcohol products, Dess-Martin periodinane $(158 \mathrm{mg}, 374 \mu \mathrm{mol})$ and dichloromethane $(10 \mathrm{~mL})$ were added to a flask and stirred at room temperature for $1 \mathrm{~h}$. The reaction was then evaporated on to silica under reduced pressure and purified by column chromatography using diethyl ether : hexane (3:7) to afford (3-methyl-2-(3-methylbut-2-en-1-yl)-6-phenylcyclohex-3-en-1-yl) (4-methoxy-2,6bis(methoxymethoxy)phenyl) methanone $\mathbf{2 0}$ as colorless oil (85.0 $\mathrm{mg}, 69 \%$ over 2 steps); $\mathbf{R}_{\mathbf{f}}$ (diethyl ether:hexane 1:1) 0.39; ${ }^{1} \mathbf{H}-\mathbf{N M R}\left(400 \mathrm{MHz}, \mathrm{CDCl}_{3}\right) \delta$ 7.23-7.08 (5H, m, H2"', H3"', H4"'), 6.37 (2H, s, H3), 5.48 (1H, s, H3'), 5.06-4.98 (4H, m, $\left.\underline{\mathrm{CH}}_{2} \mathrm{OCH}_{3}\right), 4.87$ (1H, t, $\left.J=6.4 \mathrm{~Hz}, \mathrm{H} 2 "\right)$, 
$3.79(3 \mathrm{H}, \mathrm{s}, \mathrm{OMe}), 3.71(1 \mathrm{H}, \mathrm{dd}, J=16.0 \& 7.2 \mathrm{~Hz}, \mathrm{H} 6 '), 3.47-3.42\left(7 \mathrm{H}, \mathrm{m}, \mathrm{H} 1^{\prime} \& \mathrm{CH}_{2} \mathrm{OCH}_{3}\right)$, 2.35-2.32 (3H, m, H2' \& H5'), 2.04-1.90 (2H, m, H1"), 1.74 (3H, s, $\left.\mathrm{CH}_{3}\right), 1.55$ (3H, s, $\left.\mathrm{CH}_{3}\right), 1.25$ $\left(3 \mathrm{H}, \mathrm{s}, \mathrm{CH}_{3}\right) ;{ }^{13} \mathbf{C}-\mathbf{N M R}\left(100 \mathrm{MHz}, \mathrm{CDCl}_{3}\right) \delta 204.8,162.0,156.2,143.2,137.2,130.9,129.0,127.6$, 125.6, 123.6, 120.6, 115.1, 94.8, 94.7, 56.2, 55.4, 48.4, 45.5, 43.5, 27.8, 27.0, 25.7, 22.7, 17.5; IR (thin film) $v_{\max }=2960(\mathrm{w}), 2913(\mathrm{w}), 1698(\mathrm{~m}), 1606(\mathrm{~s}), 1452(\mathrm{w}), 1153(\mathrm{~s}), 1048(\mathrm{~s}), 924(\mathrm{w})$ $\mathrm{cm}^{-1}$; LRMS $\left(\mathrm{EI}^{+}\right) \mathrm{m} / \mathrm{z}=494\left(\mathrm{M}^{+\bullet}, 2 \%\right), 255(100 \%)$; HRMS $\left(\mathrm{EI}^{+}\right)$calcd for $\mathrm{C}_{30} \mathrm{H}_{38} \mathrm{O}_{6}\left(\mathrm{M}^{+\bullet}\right)$ 494.2668, found 494.2673.

\section{( \pm -Nicolaioidesin B (5) ${ }^{[10]}$}

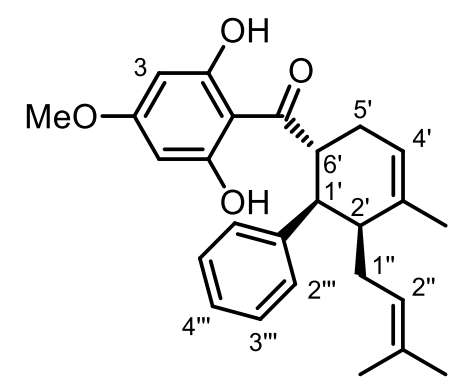

(3-Methyl-2-(3-methylbut-2-en-1-yl)-6-phenylcyclohex-3-en-1-yl) (4-methoxy-2,6-bis(methoxymethoxy)phenyl) methanone $20(71.3 \mathrm{mg}, 175 \mu \mathrm{mol})$, methanol $(10 \mathrm{~mL})$ and 4 drops of concentrated hydrochloric acid were added to a flask and heated at $50^{\circ} \mathrm{C}$ with stirring for $2 \mathrm{~h}$. The reaction mixture was then neutralized with saturated sodium bicarbonate solution, extracted with ethyl acetate $(2 \times 20 \mathrm{~mL})$ and dried over magnesium sulfate. Filtration, concentration under reduced pressure and purification via silica gel chromatography using diethyl ether : hexane (35:65) afforded $\left( \pm\right.$ )-nicolaioidesin B 5 as a pale yellow oil (49.1 mg, 69\%); $\mathbf{R}_{\mathbf{f}}$ (diethyl ether:hexane 2:3) 0.07; 'H-NMR (400 MHz, CD $\left.{ }_{3} \mathrm{OD}\right) \delta$ 7.18-7.11 (4H, m, H2'" \& H3'"), 7.07 (1H, m, H4"'), 5.93 (2H, s, H3), 5.49 (1H, s, H4'), 4.92 (1H, m, H6'), 4.88 (1H, m, H2"), 3.77 (3H, s, OMe), 3.51 (1H, dd, $J=11.6$ \& $\left.5.2 \mathrm{~Hz}, \mathrm{H} 1^{\prime}\right), 2.61$ (1H, m, H5'a), 2.24 (1H, m, H2'), 2.07-1.83 (3H, m, H5'b \& H1"), $1.75\left(3 \mathrm{H}, \mathrm{s}, \mathrm{CH}_{3}\right), 1.57\left(3 \mathrm{H}, \mathrm{s}, \mathrm{CH}_{3}\right), 1.34\left(3 \mathrm{H}, \mathrm{s}, \mathrm{CH}_{3}\right) ;{ }^{13} \mathbf{C}-\mathbf{N M R}\left(100 \mathrm{MHz}, \mathrm{CD}_{3} \mathrm{OD}\right) \delta 211.2$, $167.4,165.5,144.9,138.6,131.4,129.4,129.0,126.7,124.8,121.4,106.8,94.5,55.8,47.6,47.0$, 44.6, 31.9, 29.0, 26.1, 23.3, 18.0; IR (thin film) $v_{\max }=3292(\mathrm{w}), 2964$ (w), 2913 (w), 1625 (vs), 1575 (s), 1377 (w), 1211 (s), 1079 (w) cm ${ }^{-1}$; LRMS (ESI $\left.{ }^{-}\right) m / z=405$ ([M-H] $\left.]^{-}, 40 \%\right), 113$ (100\%); HRMS (ESI $\left.{ }^{-}\right)$calcd for $\mathrm{C}_{26} \mathrm{H}_{29} \mathrm{O}_{4}\left([\mathrm{M}-\mathrm{H}]^{-}\right)$405.2066, found 405.2067. 


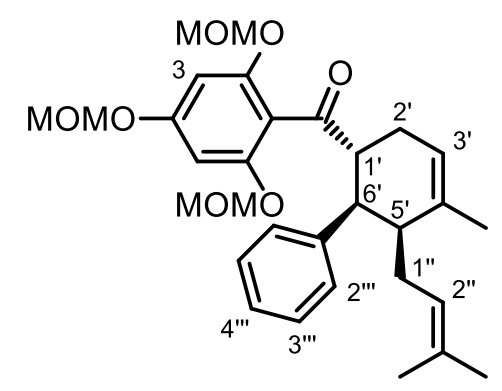

2-Bromo-1,3,5-tris(methoxymethoxy)benzene 15 (136 mg, $406 \mu \mathrm{mol})$ and tetrahydrofuran $(10 \mathrm{~mL})$ were added to a flask and cooled to $-78^{\circ} \mathrm{C}$ under a nitrogen atmosphere. Butyl lithium $(162 \mu \mathrm{L}, 1.6$ M, $406 \mu \mathrm{mol}$ ) was then added dropwise over $5 \mathrm{~min}$ and the resulting solution stirred for $30 \mathrm{~min}$. A solution of (4-methyl-5-(3-methylbut-2-en-1-yl)-6-phenylcyclohex-3-ene-1-yl)methanal 19 (36.3 $\mathrm{mg}, 135 \mu \mathrm{mol})$ in tetrahydrofuran $(5 \mathrm{~mL})$ was then added dropwise over $5 \mathrm{~min}$ followed by stirring at $-78^{\circ} \mathrm{C}$ for $15 \mathrm{~min}$. After this the reaction was allowed to warm to room temperature and stirred for a further $30 \mathrm{~min}$ followed by quenching with a saturated solution of ammonium chloride (30 $\mathrm{mL})$. Extraction with diethyl ether $(3 \times 20 \mathrm{~mL})$, drying over magnesium sulfate and purification by column chromatography using diethyl ether : hexane (1:3) )gave a mixture of the alcohol diastereomer products; $\mathbf{R}_{\mathbf{f}}$ (diethyl ether:hexane 1:1) $0.11 \&$ 0.19; The alcohol products, DessMartin periodinane $(83.2 \mathrm{mg}, 196 \mu \mathrm{mol})$ and dichloromethane $(5 \mathrm{~mL})$ were added to a flask and stirred at room temperature for $1 \mathrm{~h}$. The reaction was then evaporated on to silica under reduced pressure and purified by column chromatography using diethyl ether : hexane (3:7) to afford (4methyl-5-(3-methylbut-2-en-1-yl)-6-phenylcyclohex-3-en-1-yl) (2,4,6-tris(methoxymethoxy)phenyl) methanone 21 as colorless oil (50.3 mg, 71\% over 2 steps); $\mathbf{R}_{\mathbf{f}}$ (diethyl ether:hexane 2:3) 0.17; ${ }^{\mathbf{1}} \mathbf{H}$ NMR (400 MHz, $\left.\mathrm{CDCl}_{3}\right) \delta$ 7.24-7.16 (4H, m, H2'" \& H3"'), 7.11 (1H, m, H4"'), 6.50 (2H, s, H3), $5.47\left(1 \mathrm{H}, \mathrm{s}, \mathrm{H} 3{ }^{\prime}\right), 5.15\left(2 \mathrm{H}, \mathrm{s}, \underline{\mathrm{CH}}_{2} \mathrm{OCH}_{3}\right), 5.07-4.99\left(4 \mathrm{H}, \mathrm{m}, \underline{\mathrm{CH}}_{2} \mathrm{OCH}_{3}\right), 4.87\left(1 \mathrm{H}, \mathrm{m}, \mathrm{H} 2^{\prime \prime}\right), 3.69$ $(1 \mathrm{H}, \mathrm{dd}, J=8.4 \& 7.2 \mathrm{~Hz}, \mathrm{H} 6 '), 3.50-3.42\left(10 \mathrm{H}, \mathrm{m}, \mathrm{H} 1^{\prime} \& \mathrm{CH}_{2} \mathrm{OCH}_{3}\right), 2.35-2.34$ (3H, m, H2' \& H5'), 2.04-1.90 (2H, m, H1"), $1.75\left(3 \mathrm{H}, \mathrm{s}, \mathrm{CH}_{3}\right), 1.55\left(3 \mathrm{H}, \mathrm{s}, \mathrm{CH}_{3}\right), 1.24\left(3 \mathrm{H}, \mathrm{s}, \mathrm{CH}_{3}\right) ;{ }^{13} \mathbf{C}-\mathbf{N M R}$ $\left(100 \mathrm{MHz}, \mathrm{CDCl}_{3}\right) \delta 204.7,159.6,155.9,143.2,137.3,131.0,129.0,127.6,125.6,123.6,120.5$, 116.0, 96.8, 94.7, 94.4, $56.3(2 \times \mathrm{C}), 48.5,45.3,43.4,27.8,26.8,25.7,22.7,17.5$; IR (thin film) $v_{\max }=2959(\mathrm{~m}), 2912(\mathrm{~m}), 1700(\mathrm{~m}), 1605(\mathrm{~s}), 1451(\mathrm{~m}), 1216(\mathrm{~m}), 1155(\mathrm{~s}), 1050(\mathrm{~s}) \mathrm{cm}^{-1}$; LRMS $\left(\mathrm{EI}^{+}\right) m / z=524\left(\mathrm{M}^{+\bullet}, 1 \%\right), 285(100 \%)$; HRMS $\left(\mathrm{EI}^{+}\right)$calcd for $\mathrm{C}_{31} \mathrm{H}_{40} \mathrm{O}_{7}\left(\mathrm{M}^{+\bullet}\right) 524.2774$, found 524.2790 . 
(土)-4-Hydroxyisopanduratin A $(6)^{[11]}$

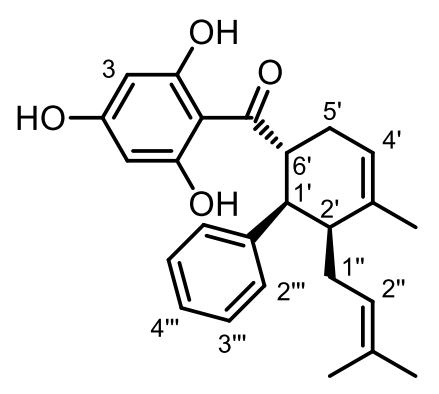

(3-Methyl-2-(3-methylbut-2-enyl)-6-phenylcyclohex-3-enyl)(2,4,6-tris(methoxymethoxy)phenyl)methanone $21(40.0 \mathrm{mg}, 76.2 \mu \mathrm{mol})$, methanol $(10 \mathrm{~mL})$ and 4 drops of concentrated hydrochloric acid were added to a flask and heated at $50^{\circ} \mathrm{C}$ with stirring for $2 \mathrm{~h}$. The reaction mixture was then neutralized with saturated sodium bicarbonate solution, extracted with ethyl acetate $(2 \times 20 \mathrm{~mL})$ and dried over magnesium sulfate. Filtration, concentration under reduced pressure and purification via silica gel chromatography using diethyl ether : hexane (35:65) afforded ( \pm )-4hydroxyisopanduratin A 6 as a pale yellow oil (21.1 mg, 71\%); $\mathbf{R}_{\mathbf{f}}$ (diethyl ether:hexane 2:3) 0.07; ${ }^{1}$ H-NMR (800 MHz, CD 3 OD) $\delta$ 7.19-7.06 (5H, m, H2'", H3"' \& H4"'), 5.81 (2H, s, H3), 5.49 (1H, s, H4'), 4.92 (1H, ddd, $J=11.1,11.1 \& 6.1$ Hz, H6'), 4.88 (1H, obscured by HOD, H2"), 3.50 (1H, dd, $\left.J=11.7 \& 5.0 \mathrm{~Hz}, \mathrm{H} 1^{\prime}\right), 2.60$ (1H, m, H5' $\beta$ ), 2.24 (1H, m, H2'), 2.05-1.96 (2H, m, H1"), 1.86 (1H, m, $\left.5^{\prime} \alpha\right), 1.75\left(3 \mathrm{H}, \mathrm{s}, \mathrm{CH}_{3}\right), 1.57\left(3 \mathrm{H}, \mathrm{s}, \mathrm{CH}_{3}\right), 1.34\left(3 \mathrm{H}, \mathrm{s}, \mathrm{CH}_{3}\right) ;{ }^{13} \mathbf{C}-\mathbf{N M R}\left(200 \mathrm{MHz}, \mathrm{CD}_{3} \mathrm{OD}\right) \delta$ 210.8, 166.1, 145.0, 138.6, 131.4, 129.5, 129.0, 126.7, 124.9, 121.5, 106.1, 95.9 (2 × C), 47.6, 47.1, 44.4, 31.9, 29.1, 26.1, 23.3, 17.9; IR (thin film) $v_{\max }=3235$ (w), $2918(w), 2853(w), 1627(\mathrm{~s})$, 1599 (s), 1451 (m), 1202 (m), 1073 (m) cm ${ }^{-1}$; LRMS (ESI $\left.{ }^{-}\right) m / z=392\left([\mathrm{M}-\mathrm{H}]^{-}, 10 \%\right), 153$ (100\%); HRMS (ESI $\left.{ }^{-}\right)$calcd for $\mathrm{C}_{25} \mathrm{H}_{27} \mathrm{O}_{4}\left([\mathrm{M}-\mathrm{H}]^{-}\right)$391.1909, found 391.1899. 
Panduratin H (3) ${ }^{1} \mathrm{H}$ NMR Comparison.

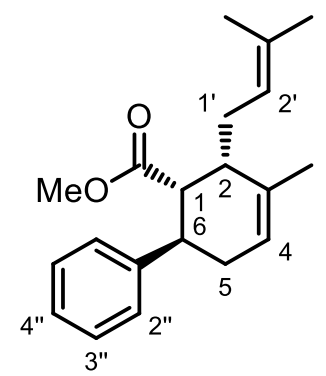

\begin{tabular}{cccc}
\hline Proton & $\delta_{\mathrm{L}}\left(\mathrm{Lit}_{\text {. }}{ }^{[3]} 400 \mathrm{MHz}, \mathrm{CDCl}_{3}\right)$ & $\delta_{3}\left(\right.$ Compound $\left.3,400 \mathrm{MHz}, \mathrm{CDCl}_{3}\right)$ & $\Delta \delta\left(\delta_{\mathrm{L}}-\delta_{3}\right)$ \\
\hline $\mathrm{H} 3^{\prime \prime}$ & $7.25(\mathrm{~m})$ & $7.26(\mathrm{~m})$ & -0.01 \\
$\mathrm{H}{ }^{\prime}, \mathrm{H} 4 "$ & $7.15(\mathrm{~m})$ & $7.17^{\mathrm{a}}(\mathrm{m})$ & 0.02 \\
$\mathrm{H} 4$ & $5.42(\mathrm{~s})$ & $5.42(\mathrm{~s})$ & 0.00 \\
$\mathrm{H} 2^{\prime}$ & $5.06(\mathrm{t}, 6.8)$ & $5.06(\mathrm{~m})$ & 0.00 \\
$\mathrm{OCH}_{3}$ & $3.40(\mathrm{~s})$ & $3.40(\mathrm{~s})$ & 0.00 \\
$\mathrm{H} 6$ & $3.19(\mathrm{ddd}, 11.2,10.0,6.0)$ & $3.19(\mathrm{ddd}, 12.0,10.4,6.4)$ & 0.00 \\
$\mathrm{H} 1$ & $3.09(\mathrm{dd}, 11.2,5.4)$ & $2.45(\mathrm{dd}, 12.4,4.4,5.2)$ & 0.01 \\
$\mathrm{H} 2$ & $2.45(\mathrm{dd}, 10.2,5.4)$ & $2.32^{a}(\mathrm{~m})$ & 0.00 \\
$\mathrm{H} 5 \beta$ & $2.34(\mathrm{dt}, 18.3,6.0,1.9)$ & & - \\
$\mathrm{H} 1^{\prime} \mathrm{a}$ & $2.26(\mathrm{~m})$ & $2.17(\mathrm{~m})$ & - \\
$\mathrm{H} 1^{\prime} \mathrm{b}$ & $2.18(\mathrm{~m})$ & $2.04(\mathrm{~m})$ & 0.01 \\
$\mathrm{H} 5 \alpha$ & $2.04(\mathrm{~m})$ & $1.78(\mathrm{~s})$ & 0.00 \\
$\mathrm{CH}_{3}$ & $1.77(\mathrm{~s})$ & $1.67(\mathrm{~s})$ & -0.01 \\
$\mathrm{CH}_{3}$ & $1.67(\mathrm{~s})$ & $1.60(\mathrm{~s})$ & 0.00 \\
$\mathrm{CH}_{3}$ & $1.59(\mathrm{~s})$ & & -0.01 \\
\hline
\end{tabular}

${ }^{a}$ Calculated from midpoint of multiplet. 
Panduratin H (3) ${ }^{13} \mathrm{C}$ NMR Comparison.

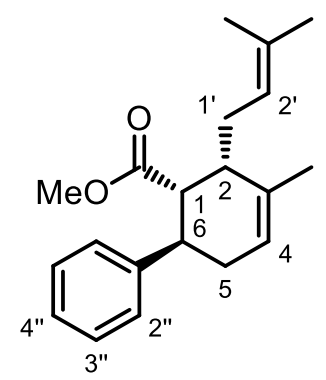

Carbon $\quad \delta_{\mathrm{L}}\left(\right.$ Lit., $\left.^{[3]} 100 \mathrm{MHz}, \mathrm{CDCl}_{3}\right) \quad \delta_{\mathbf{3}}\left(\right.$ Compound 3, $\left.100 \mathrm{MHz}, \mathrm{CDCl}_{3}\right) \quad \Delta \delta\left(\delta_{\mathrm{L}}-\delta_{\mathbf{3}}\right)$

\begin{tabular}{cccc}
\hline $\mathrm{COOCH}_{3}$ & 173.7 & 173.8 & -0.1 \\
$\mathrm{C}{ }^{\prime \prime}$ & 145.9 & 145.9 & 0.0 \\
$\mathrm{C} 3$ & 136.3 & 136.3 & 0.0 \\
$\mathrm{C}^{\prime}$ & 131.9 & 132.0 & -0.1 \\
$\mathrm{C}^{\prime \prime}, \mathrm{C}^{\prime \prime}$ & 128.3 & 128.3 & 0.0 \\
$\mathrm{C}^{\prime \prime}, \mathrm{C}^{\prime \prime}$ & 127.1 & 127.1 & 0.0 \\
$\mathrm{C} 4 "$ & 125.9 & 126.0 & -0.1 \\
$\mathrm{C}{ }^{\prime}$ & 123.0 & 123.8 & -0.8 \\
$\mathrm{C} 4$ & 121.1 & 121.0 & 0.1 \\
$\mathrm{COOC} \mathrm{H}_{3}$ & 51.0 & 51.0 & 0.0 \\
$\mathrm{C} 1$ & 49.2 & 49.1 & 0.1 \\
$\mathrm{C} 2$ & 42.7 & 42.6 & 0.1 \\
$\mathrm{C}^{\prime}$ & 37.2 & 37.1 & 0.1 \\
$\mathrm{C}^{\prime}$ & 35.1 & 35.1 & 0.0 \\
$\mathrm{C}^{\prime}$ & 29.2 & 29.2 & 0.0 \\
$\mathrm{C}^{\prime}$ & 25.9 & 25.9 & 0.0 \\
$3-\mathrm{CH}_{3}$ & 22.6 & 22.6 & 0.0 \\
$\mathrm{C}^{\prime}$ & 17.9 & 17.9 & 0.0 \\
\hline
\end{tabular}


Panduratin I (4) ${ }^{1} \mathrm{H}$ NMR Comparison.

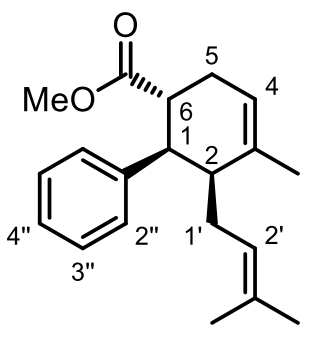

\begin{tabular}{|c|c|c|c|}
\hline Proton & $\delta_{\mathrm{L}}\left(\right.$ Lit., $\left.{ }^{\left[{ }^{[3]}\right.} 400 \mathrm{MHz}, \mathrm{CDCl}_{3}\right)$ & $\delta_{3}\left(\right.$ Compound 4, $\left.400 \mathrm{MHz}, \mathrm{CDCl}_{3}\right)$ & $\Delta \delta\left(\delta_{\mathrm{L}}-\delta_{4}\right)$ \\
\hline H3" & $7.25(\mathrm{~m})$ & $7.26(\mathrm{~m})$ & -0.01 \\
\hline H2", H4" & $7.15(\mathrm{~m})$ & $7.17^{\mathrm{a}}(\mathrm{m})$ & -0.02 \\
\hline $\mathrm{H} 4$ & $5.45(\mathrm{~s})$ & $5.45(\mathrm{~s})$ & 0.00 \\
\hline $\mathrm{H} 2^{\prime}$ & $4.80(t, 7.6)$ & $4.81(\mathrm{~m})$ & -0.01 \\
\hline $\mathrm{OCH}_{3}$ & $3.49(\mathrm{~s})$ & $3.49(\mathrm{~s})$ & 0.00 \\
\hline $\mathrm{H} 1$ & $3.33(\mathrm{dd}, 11.2,5.6)$ & $3.33(\mathrm{dd}, 11.2,5.2)$ & 0.00 \\
\hline H6 & $3.18(\mathrm{ddd}, 11.2,9.6,6.1)$ & $3.18(\mathrm{ddd}, 11.2,9.6,6.4)$ & 0.00 \\
\hline $\mathrm{H} 5 \beta$ & $2.43(\mathrm{dt}, 18.0,6.1,1.9)$ & $2.44(\mathrm{~m})$ & -0.01 \\
\hline $\mathrm{H} 5 \alpha$ & $2.34(\mathrm{~m})$ & $2.32,(\mathrm{~m})$ & 0.02 \\
\hline $\mathrm{H} 2$ & $2.21(\mathrm{dd}, 10.2,5.6)$ & $2.23(\mathrm{dd}, 10.8,5.2)$ & -0.02 \\
\hline $\mathrm{H} 1^{\prime}$ & $1.93(\mathrm{~m})$ & $1.93^{a}(\mathrm{~m})$ & 0.00 \\
\hline $\mathrm{CH}_{3}$ & $1.74(\mathrm{~s})$ & $1.74(\mathrm{~s})$ & 0.00 \\
\hline $\mathrm{CH}_{3}$ & $1.56(\mathrm{~s})$ & $1.57(\mathrm{~s})$ & -0.01 \\
\hline $\mathrm{CH}_{3}$ & $1.34(\mathrm{~s})$ & $1.34(\mathrm{~s})$ & 0.00 \\
\hline
\end{tabular}

${ }^{a}$ Calculated from midpoint of multiplet. 
Panduratin I (4) ${ }^{13} \mathrm{C}$ NMR Comparison.

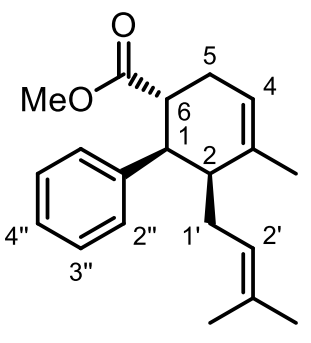

\begin{tabular}{|c|c|c|c|}
\hline Carbon & $\delta_{\mathrm{L}}\left(\right.$ Lit., $\left.^{\left[{ }^{[3]}\right.} 100 \mathrm{MHz} \mathrm{CDCl}_{3}\right)$ & $\delta_{3}\left(\right.$ Compound 3, $\left.100 \mathrm{MHz}, \mathrm{CDCl}_{3}\right)$ & $\Delta \delta\left(\delta_{\mathrm{L}}-\delta_{4}\right)$ \\
\hline $\mathrm{COOCH}_{3}$ & 176.5 & 176.5 & 0.0 \\
\hline C1" & 142.4 & 142.3 & 0.1 \\
\hline $\mathrm{C} 3$ & 137.7 & 137.6 & 0.1 \\
\hline $\mathrm{C} 3^{\prime}$ & 131.0 & 131.0 & 0.0 \\
\hline \multirow[t]{2}{*}{ C2", C4", C6" } & 128.2 & 128.2 & - \\
\hline & & 128.1 & - \\
\hline $\mathrm{C} 3 ", \mathrm{C} 5^{\prime \prime}$ & 126.2 & 126.1 & 0.1 \\
\hline $\mathrm{C} 2^{\prime}$ & 123.4 & 123.4 & 0.0 \\
\hline $\mathrm{C} 4$ & 119.5 & 119.5 & 0.0 \\
\hline $\mathrm{COOCH}_{3}$ & 51.5 & 51.5 & 0.0 \\
\hline $\mathrm{C} 1$ & 46.3 & 46.3 & 0.0 \\
\hline $\mathrm{C} 2$ & 44.6 & 44.5 & 0.1 \\
\hline C6 & 39.9 & 39.9 & 0.0 \\
\hline $\mathrm{C} 5$ & 29.5 & 29.5 & 0.0 \\
\hline $\mathrm{C} 1^{\prime}$ & 27.7 & 27.6 & 0.1 \\
\hline $\mathrm{C} 4^{\prime}$ & 25.8 & 25.8 & 0.0 \\
\hline $3-\mathrm{CH}_{3}$ & 22.9 & 22.9 & 0.0 \\
\hline $\mathrm{C} 5^{\prime}$ & 17.7 & 17.7 & 0.0 \\
\hline
\end{tabular}


Panduratin A (1) ${ }^{1} \mathrm{H}$ NMR Comparison.

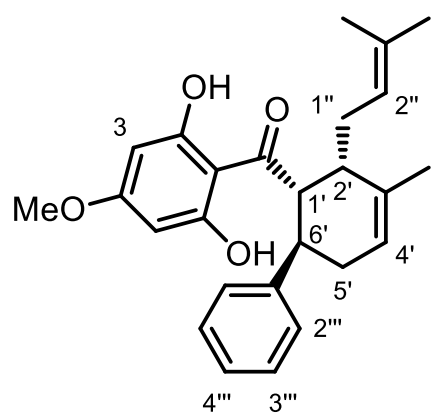

\begin{tabular}{|c|c|c|c|}
\hline Proton & $\delta_{\mathrm{L}}\left(\right.$ Lit., $\left.^{\left[{ }^{[8]}\right.} 500 \mathrm{MHz}, \mathrm{CDCl}_{3}\right)$ & $\delta_{\mathbf{3}}\left(\right.$ Compound $\left.\mathbf{1}, 400 \mathrm{MHz}, \mathrm{CD}_{3} \mathrm{OD}\right)$ & $\Delta \delta\left(\delta_{\mathrm{L}}-\delta_{1}\right)$ \\
\hline H2", H3" & $7.21(\mathrm{~m})$ & $7.17^{\mathrm{a}}(\mathrm{m})$ & 0.04 \\
\hline H4" & $7.10(\mathrm{~m})$ & $7.05(\mathrm{~m})$ & 0.05 \\
\hline $\mathrm{H} 3$ & $5.87(\mathrm{~s})$ & $5.88(\mathrm{~s})$ & -0.01 \\
\hline $\mathrm{H} 4^{\prime}$ & $5.43(\mathrm{~s})$ & $5.41(\mathrm{~s})$ & 0.02 \\
\hline $\mathrm{H} 2 "$ & $4.87(\mathrm{t}, 6.7)$ & $4.88^{b}$ & -0.01 \\
\hline $\mathrm{H} 1^{\prime}$ & $4.66(\mathrm{dd}, 11.3,4.6)$ & $4.77(\mathrm{dd}, 11.6,4.4)$ & -0.11 \\
\hline $\mathrm{OMe}$ & $3.74(\mathrm{~s})$ & $3.74(\mathrm{~s})$ & 0.00 \\
\hline $\mathrm{H} 6^{\prime}$ & $3.43(\mathrm{ddd}, 11.0,11.0,6.3)$ & $3.38(\mathrm{ddd}, 10.8,10.8,6.4)$ & 0.05 \\
\hline $\mathrm{H} 2^{\prime}$ & $2.63(\mathrm{~m})$ & $2.65(\mathrm{~m})$ & -0.02 \\
\hline H5'a, H1"a & $2.40(\mathrm{~m})$ & $2.30^{a}(\mathrm{~m})$ & 0.10 \\
\hline $\mathrm{H} 5^{\prime} \mathrm{b}, \mathrm{H} 11^{\prime \prime} \mathrm{b}$ & $2.03(\mathrm{~m})$ & $2.02^{a}(\mathrm{~m})$ & 0.01 \\
\hline $\mathrm{CH}_{3}$ & $1.78(\mathrm{~s})$ & $1.77(\mathrm{~s})$ & 0.01 \\
\hline $\mathrm{CH}_{3}$ & $1.52(\mathrm{~s})$ & $1.50(\mathrm{~s})$ & 0.02 \\
\hline
\end{tabular}

${ }^{a}$ Calculated from midpoint of multiplet.

${ }^{b}$ Obscured by HOD peak, chemical shift determined from COSY. 
Panduratin A (1) ${ }^{13} \mathrm{C}$ NMR Comparison.

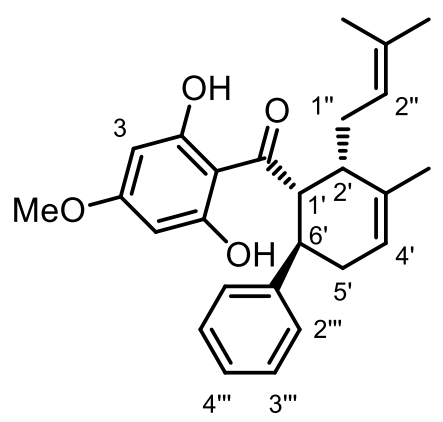

\begin{tabular}{|c|c|c|c|}
\hline Carbon & $\delta_{\mathrm{L}}\left(\right.$ Lit., $\left.{ }^{[8]} 125 \mathrm{MHz}, \mathrm{CDCl}_{3}\right)$ & $\delta_{\mathbf{3}}\left(\right.$ Compound $\left.\mathbf{1}, 100 \mathrm{MHz}, \mathrm{CD}_{3} \mathrm{OD}\right)$ & $\Delta \delta\left(\delta_{\mathrm{L}}-\delta_{1}\right)$ \\
\hline $\mathrm{C}=\mathrm{O}$ & 206.4 & 208.1 & -1.7 \\
\hline $\mathrm{C} 2$ & 165.0 & 167.0 & -2.0 \\
\hline $\mathrm{C} 4$ & 165.0 & 165.4 & -0.4 \\
\hline $\mathrm{C} 1^{\prime \prime \prime}$ & 147.0 & 148.5 & -1.5 \\
\hline $\mathrm{C} 3^{\prime}$ & 137.1 & 138.4 & -1.3 \\
\hline C3" & 131.9 & 132.4 & -0.5 \\
\hline $\mathrm{C} 3^{\prime \prime \prime}$ & 128.3 & 129.2 & -0.9 \\
\hline $\mathrm{C} 2^{\prime \prime \prime}$ & 127.0 & 128.3 & -1.3 \\
\hline $\mathrm{C} 4{ }^{\prime \prime \prime}$ & 125.6 & 126.4 & -0.6 \\
\hline $\mathrm{C} 2 "$ & 124.2 & 125.7 & -1.5 \\
\hline $\mathrm{C} 4^{\prime}$ & 121.1 & 122.0 & -0.9 \\
\hline $\mathrm{C} 1$ & 105.7 & 107.2 & -1.5 \\
\hline $\mathrm{C} 3$ & 94.5 & 94.4 & 0.1 \\
\hline $\mathrm{OMe}$ & 55.4 & 55.8 & -0.4 \\
\hline $\mathrm{C} 1^{\prime}$ & 53.9 & 55.0 & -1.1 \\
\hline $\mathrm{C} 2^{\prime}$ & 42.6 & 43.4 & -0.6 \\
\hline $\mathrm{C} 6^{\prime}$ & 37.0 & 38.4 & -1.4 \\
\hline $\mathrm{C} 5^{\prime}$ & 35.9 & 37.1 & -1.2 \\
\hline $\mathrm{C} 1 "$ & 28.8 & 29.8 & -1.0 \\
\hline $9^{\prime}-\mathrm{CH}_{3}$ & 25.6 & 25.9 & -0.3 \\
\hline $3^{\prime}-\mathrm{CH}_{3}$ & 22.7 & 23.0 & -0.3 \\
\hline $9^{\prime}-\mathrm{CH}_{3}$ & 17.9 & 18.1 & -0.2 \\
\hline
\end{tabular}


4-Hydroxypanduratin A (2) ${ }^{1} \mathrm{H}$ NMR Comparison.

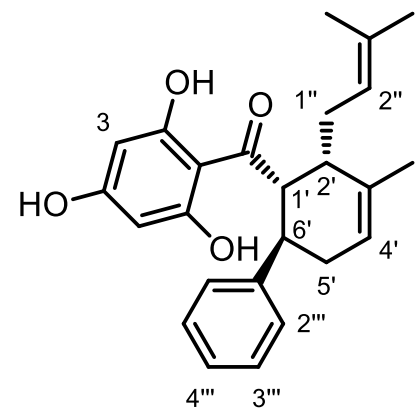

\begin{tabular}{|c|c|c|c|}
\hline Proton & $\delta_{\mathrm{L}}\left(\right.$ Lit., $\left.{ }^{[9]} 600 \mathrm{MHz}, \mathrm{CD}_{3} \mathrm{OD}\right)$ & $\delta_{3}\left(\right.$ Compound $\left.2,400 \mathrm{MHz}, \mathrm{CD}_{3} \mathrm{OD}\right)$ & $\Delta \delta\left(\delta_{\mathrm{L}}-\delta_{2}\right)$ \\
\hline H2", H3" & $7.17(\mathrm{~m})$ & $7.18^{\mathrm{a}}(\mathrm{m})$ & -0.01 \\
\hline H4" & $7.05(\mathrm{~m})$ & $7.05(\mathrm{~m})$ & 0.00 \\
\hline $\mathrm{H} 3$ & $5.76(\mathrm{~s})$ & $5.78(\mathrm{~s})$ & -0.02 \\
\hline $\mathrm{H} 4^{\prime}$ & 5.41 (ddd, $4.5,2.8,1.9)$ & $5.40(\mathrm{~s})$ & 0.01 \\
\hline $\mathrm{H} 2 "$ & $4.90(\mathrm{dd}, 7.1,7.1)$ & $4.91^{\mathrm{b}}$ & -0.01 \\
\hline $\mathrm{H} 1^{\prime}$ & $4.75(\mathrm{dd}, 11.6,4.7)$ & $4.76(\mathrm{dd}, 12.0,4.8)$ & -0.01 \\
\hline $\mathrm{H} 6^{\prime}$ & $3.36(\mathrm{ddd}, 11.6,10.6,6.4)$ & $3.37(\mathrm{ddd}, 10.4,10.4,6.4)$ & -0.01 \\
\hline $\mathrm{H} 2^{\prime}$ & 2.64 (dddd, $7.2,4.7,4.5,1.6)$ & $2.65(\mathrm{~m})$ & -0.01 \\
\hline $\mathrm{H} 5^{\prime} \mathrm{a}$ & 2.33 (dddq, $18.1,6.4,4.5,1.9)$ & $2.29^{\mathrm{a}}(\mathrm{m})$ & - \\
\hline $\mathrm{H} 1 " \mathrm{a}$ & $2.25(\mathrm{ddd}, 15.2,7.2,7.1)$ & & - \\
\hline H1b" & $2.05(\mathrm{ddd}, 15.2,7.1,4.5)$ & & - \\
\hline $\mathrm{H} 5 \mathrm{~s}^{\prime} \mathrm{b}$ & 1.98 (ddddq, 10.6, 2.8, 1.9, 1.6) & $2.01(\mathrm{~m})$ & -0.03 \\
\hline $\mathrm{CH}_{3}$ & $1.77(\mathrm{ddd}, 1.9,1.9,1.9)$ & $1.77(\mathrm{~s})$ & 0.00 \\
\hline $\mathrm{CH}_{3}$ & $1.51(\mathrm{~s})$ & $1.51(\mathrm{~s})$ & 0.00 \\
\hline
\end{tabular}

${ }^{a}$ Calculated from midpoint of multiplet.

${ }^{b}$ Obscured by HOD peak, chemical shift determined from COSY. 
4-Hydroxypanduratin A (2) ${ }^{13} \mathrm{C}$ NMR Comparison.

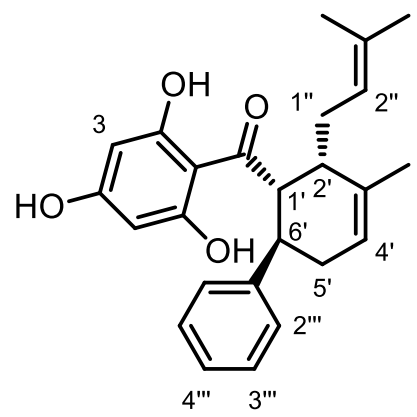

\begin{tabular}{|c|c|c|c|}
\hline Carbon & $\delta_{\mathrm{L}}\left(\right.$ Lit.. $^{[9]} 150 \mathrm{MHz}$, DMSO- $\left.d_{6}\right)$ & $\delta_{\mathbf{3}}\left(\right.$ Compound $\left.2,100 \mathrm{MHz}, \mathrm{CD}_{3} \mathrm{OD}\right)$ & $\Delta \delta\left(\delta_{\mathrm{L}}-\delta_{2}\right)$ \\
\hline $\mathrm{C}=\mathrm{O}$ & 205.7 & 207.8 & -2.1 \\
\hline $\mathrm{C} 4$ & 164.3 & 165.5 & - \\
\hline $\mathrm{C} 2$ & 164.1 & & - \\
\hline $\mathrm{C} 1^{\prime \prime \prime}$ & $a$ & 148.6 & - \\
\hline $\mathrm{C} 1 "$ & $137.1^{b}$ & 138.4 & -1.3 \\
\hline C3" & 130.6 & 132.2 & -1.6 \\
\hline C3"' & 128.1 & 129.1 & -1.0 \\
\hline $\mathrm{C} 2$ '" & 126.9 & 128.2 & -1.3 \\
\hline $\mathrm{C} 4{ }^{\prime \prime \prime}$ & 125.3 & 126.4 & -1.1 \\
\hline $\mathrm{C} 2 "$ & 124.3 & 125.7 & -1.4 \\
\hline $\mathrm{C} 4^{\prime}$ & 120.9 & 121.9 & -1.0 \\
\hline $\mathrm{C} 1$ & 104.7 & 106.5 & -1.8 \\
\hline $\mathrm{C} 3$ & 94.8 & 95.8 & -1.0 \\
\hline $\mathrm{C} 1^{\prime}$ & 52.8 & 54.8 & -2.0 \\
\hline $\mathrm{C} 2^{\prime}$ & 42.1 & 43.8 & -1.7 \\
\hline $\mathrm{C} 6^{\prime}$ & 36.4 & 38.4 & -2.0 \\
\hline $\mathrm{C}^{\prime}{ }^{\prime}$ & 35.7 & 37.1 & -1.4 \\
\hline C1" & 28.4 & 29.9 & -1.5 \\
\hline $\mathrm{CH}_{3}$ & 25.5 & 25.9 & -0.4 \\
\hline $\mathrm{CH}_{3}$ & 22.6 & 23.0 & -0.4 \\
\hline $\mathrm{CH}_{3}$ & 17.6 & 18.0 & -0.4 \\
\hline
\end{tabular}

${ }^{a}$ Not reported in isolation paper

${ }^{b}$ Reported as 7.1 and assumed to be a typographical error 
Nicolaioidesin B (5) ${ }^{1} \mathrm{H}$ NMR Comparison.

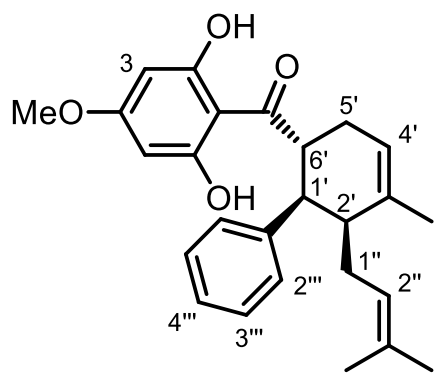

\begin{tabular}{cccc}
\hline Proton & $\delta_{\mathrm{L}}\left(\mathrm{Lit}_{\text {. }}{ }^{[10]} 500 \mathrm{MHz}, \mathrm{CDCl}_{3}\right)$ & $\delta_{\mathbf{3}}$ (Compound $\left.\mathbf{5}, 400 \mathrm{MHz}, \mathrm{CD}_{3} \mathrm{OD}\right)$ & $\Delta \delta\left(\delta_{\mathrm{L}}-\delta_{\mathbf{5}}\right)$ \\
\hline $\mathrm{H}$ & $7.16^{\mathrm{a}}(\mathrm{m})$ & $7.15^{\mathrm{a}}(\mathrm{m})$ & 0.01 \\
$\mathrm{H}$ & $7.08(\mathrm{~m})$ & $7.07(\mathrm{~m})$ & 0.01 \\
$\mathrm{H} 3$ & $5.92(\mathrm{~s})$ & $5.93(\mathrm{~s})$ & -0.01 \\
$\mathrm{H} 4^{\prime}$ & $5.49(\mathrm{~s})$ & $5.49(\mathrm{~s})$ & 0.00 \\
$\mathrm{H} 6{ }^{\prime}$ & $4.86(\mathrm{t}, 5.9)$ & $4.92(\mathrm{~m})$ & -0.06 \\
$\mathrm{H} 2^{\prime \prime}$ & $4.76(\mathrm{ddd}, 11.0,11.0,6.1)$ & $4.88^{\mathrm{b}}(\mathrm{m})$ & -0.12 \\
$\mathrm{OMe}$ & $3.76(\mathrm{~s})$ & $3.77(\mathrm{~s})$ & -0.01 \\
$\mathrm{H} 1^{\prime}$ & $3.57(\mathrm{dd}, 11.0,5.1)$ & $3.51(\mathrm{dd}, 11.6,5.2)$ & 0.06 \\
$\mathrm{H} 5^{\prime} \mathrm{a}$ & $2.62(\mathrm{dt}, 18.0,4.2)$ & $2.61(\mathrm{~m})$ & 0.01 \\
$\mathrm{H} 2^{\prime}$ & $2.27(\mathrm{dt}, 5.0,4.9)$ & $2.24(\mathrm{~m})$ & 0.03 \\
$\mathrm{H} 1^{\prime \prime}, \mathrm{H}^{\prime} \mathrm{b}$ & $1.97(\mathrm{~m})$ & $1.95^{\mathrm{a}}(\mathrm{m})$ & 0.02 \\
$3-\mathrm{CH}_{3}$ & $1.76(\mathrm{~s})$ & $1.75(\mathrm{~s})$ & 0.01 \\
$3^{\prime \prime}-\mathrm{CH}_{3}$ & $1.56(\mathrm{~s})$ & $1.57(\mathrm{~s})$ & -0.01 \\
$3^{\prime \prime}-\mathrm{CH}_{3}$ & $1.33(\mathrm{~s})$ & $1.34(\mathrm{~s})$ & -0.01 \\
\hline
\end{tabular}

${ }^{a}$ Calculated from midpoint of multiplet.

${ }^{b}$ Obscured by HOD peak, chemical shift determined from COSY. 
Nicolaioidesin B (5) ${ }^{13} \mathrm{C}$ NMR Comparison.

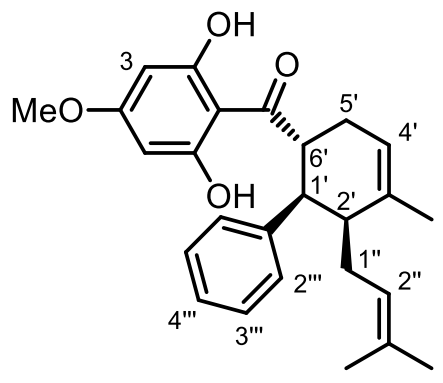

\begin{tabular}{|c|c|c|c|}
\hline Carbon & $\delta_{\mathrm{L}}\left(\right.$ Lit.. $\left.^{[10]} 125 \mathrm{MHz}, \mathrm{CDCl}_{3}\right)$ & $\delta_{\mathbf{3}}\left(\right.$ Compound $\left.\mathbf{5}, 100 \mathrm{MHz}, \mathrm{CD}_{3} \mathrm{OD}\right)$ & $\Delta \delta\left(\delta_{L}-\delta_{5}\right)$ \\
\hline $\mathrm{C}=\mathrm{O}$ & 209.2 & 211.2 & -2.0 \\
\hline 4 & 165.4 & 167.4 & -2.0 \\
\hline 2 & 162.7 & 165.5 & -2.8 \\
\hline $1^{\prime \prime \prime}$ & 143.4 & 144.9 & -1.5 \\
\hline $3^{\prime}$ & 137.6 & 138.6 & -1.0 \\
\hline $3^{\prime \prime}$ & 130.4 & 131.4 & -1.0 \\
\hline $2^{\prime \prime \prime}$ & 128.1 & 129.4 & -1.3 \\
\hline 3"' & 128.1 & 129.0 & -0.9 \\
\hline $4^{\prime \prime \prime}$ & 125.7 & 126.7 & -1.0 \\
\hline $2^{\prime \prime}$ & 123.8 & 124.8 & -1.0 \\
\hline $4^{\prime}$ & 120.1 & 121.4 & -1.3 \\
\hline 1 & 105.2 & 106.8 & -1.6 \\
\hline 3 & 94.6 & 94.5 & -0.9 \\
\hline $\mathrm{OMe}$ & 55.5 & 55.8 & -0.3 \\
\hline $1^{\prime}$ & 46.0 & 47.6 & -1.6 \\
\hline $2^{\prime}$ & 45.6 & 47.0 & -1.4 \\
\hline $6^{\prime}$ & 43.8 & 44.6 & -0.8 \\
\hline $5^{\prime}$ & 30.9 & 31.9 & -1.0 \\
\hline $1^{\prime \prime}$ & 28.0 & 29.0 & -1.0 \\
\hline $3 "-\mathrm{CH}_{3}$ & 25.9 & 26.1 & -0.2 \\
\hline $3^{\prime}-\mathrm{CH}_{3}$ & 22.9 & 23.3 & -0.4 \\
\hline 3"- $-\mathrm{CH}_{3}$ & 17.8 & 18.0 & -0.2 \\
\hline
\end{tabular}


4-Hydroxyisopanduratin A (6) ${ }^{1} \mathrm{H}$ NMR Comparison.

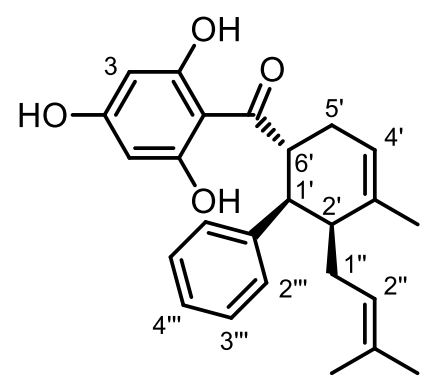

\begin{tabular}{|c|c|c|c|}
\hline Proton & $\delta_{\mathrm{L}}\left(\right.$ Lit.. $\left.{ }^{[1]]} 400 \mathrm{MHz}, \mathrm{CD}_{3} \mathrm{OD}\right)$ & $\delta_{3}\left(\right.$ Compound $\left.6,800 \mathrm{MHz}, \mathrm{CD}_{3} \mathrm{OD}\right)$ & $\Delta \delta\left(\delta_{\mathrm{L}}-\delta_{6}\right)$ \\
\hline $\mathrm{H} 2^{\prime \prime \prime}$ & $7.16^{a}(\mathrm{~m})$ & $7.17^{a}(\mathrm{~m})$ & -0.01 \\
\hline H3"', H4"' & $7.06(\mathrm{~m})$ & $7.07(\mathrm{~m})$ & -0.01 \\
\hline H3 & $5.80(\mathrm{~s})$ & $5.81(\mathrm{~s})$ & -0.01 \\
\hline $\mathrm{H} 4^{\prime}$ & $5.47(\mathrm{~s})$ & $5.49(\mathrm{~s})$ & -0.02 \\
\hline $\mathrm{H} 2 "$ & $4.80(t, 7.0)$ & $4.91(\mathrm{~m})$ & -0.11 \\
\hline H6' & $4.77(\mathrm{ddd}, 11.9,11.9,6.0)$ & $4.87^{b}$ & -0.10 \\
\hline $\mathrm{H} 1^{\prime}$ & $3.49(\mathrm{dd}, 11.9,5.1)$ & $3.50(\mathrm{dd}, 11.7,5.0)$ & -0.01 \\
\hline $\mathrm{H} 5^{\prime} \mathrm{b}$ & $2.61(\mathrm{ddd}, 17.3,6.0,1.9)$ & $2.60(\mathrm{~d}, 17.3)$ & 0.01 \\
\hline $\mathrm{H} 2^{\prime}$ & $2.23(\mathrm{dd}, 9.8,5.1)$ & $2.24(\mathrm{~m})$ & -0.01 \\
\hline $\mathrm{H} 5^{\prime} \mathrm{a}$ & $2.04(\mathrm{ddd}, 17.3,11.9,1.9)$ & $2.00(\mathrm{~m})$ & 0.04 \\
\hline $\mathrm{H} 1 "$ & $1.94^{a}(\mathrm{~m})$ & $1.86^{a}(\mathrm{~m})$ & -0.07 \\
\hline $\mathrm{CH}_{3}$ & $1.74(\mathrm{~s})$ & $1.75(\mathrm{~s})$ & -0.01 \\
\hline $\mathrm{CH}_{3}$ & $1.57(\mathrm{~s})$ & $1.57(\mathrm{~s})$ & 0.00 \\
\hline $\mathrm{CH}_{3}$ & $1.42(\mathrm{~s})$ & $1.34(\mathrm{~s})$ & 0.08 \\
\hline
\end{tabular}

${ }^{a}$ Calculated from midpoint of multiplet.

${ }^{b}$ Obscured by HOD peak, chemical shift determined from COSY. 
4-Hydroxyisopanduratin A (6) ${ }^{13} \mathrm{C}$ NMR Comparison.

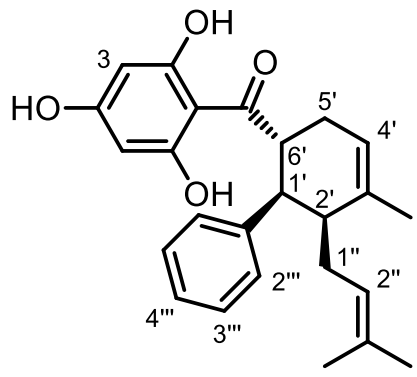

\begin{tabular}{|c|c|c|c|}
\hline Carbon & $\delta_{\mathrm{L}}\left(\right.$ Lit., $\left.{ }^{[11]} 100 \mathrm{MHz}, \mathrm{CD}_{3} \mathrm{OD}\right)$ & $\delta_{3}\left(\right.$ Compound 6 ppm, $\left.200 \mathrm{MHz}, \mathrm{CD}_{3} \mathrm{OD}\right)$ & $\Delta \delta\left(\delta_{\mathrm{L}}-\delta_{3}\right)$ \\
\hline $\mathrm{C} 7$ & 210.7 & 210.8 & -0.1 \\
\hline $\mathrm{C} 2, \mathrm{C} 4$ & 165.9 & 166.1 & -0.2 \\
\hline $\mathrm{C} 1 " '$ & 144.9 & 145.0 & -0.1 \\
\hline $\mathrm{C} 3^{\prime}$ & 138.5 & 138.6 & -0.1 \\
\hline C3" & 131.3 & 131.4 & -0.1 \\
\hline C3"' & 129.4 & 129.5 & -0.1 \\
\hline $\mathrm{C} 2$ "' & 128.9 & 129.0 & -0.1 \\
\hline C4"' & 126.6 & 126.7 & -0.1 \\
\hline C2" & 124.9 & 124.9 & 0.0 \\
\hline $\mathrm{C} 4^{\prime}$ & 121.4 & 121.5 & -0.1 \\
\hline $\mathrm{C} 1$ & 106.1 & 106.1 & 0.0 \\
\hline $\mathrm{C} 3$ & 95.9 & 95.9 & 0.0 \\
\hline $\mathrm{C} 1^{\prime}$ & 47.5 & 47.6 & -0.1 \\
\hline $\mathrm{C} 2^{\prime}$ & 47.0 & 47.1 & -0.1 \\
\hline C6' & 44.4 & 44.4 & 0.0 \\
\hline $\mathrm{C}^{\prime}$ & 31.9 & 31.9 & 0.0 \\
\hline C1" & 29.1 & 29.1 & 0.0 \\
\hline C4" & 26.1 & 26.1 & 0.0 \\
\hline $3^{\prime} \mathrm{CH}_{3}$ & 23.3 & 23.3 & 0.0 \\
\hline C5" & 17.9 & 17.9 & 0.0 \\
\hline
\end{tabular}




\section{Computational Details}

The geometries of all compounds were optimized at the B3LYP/6-31+G(d) level of theory. Systematic conformer searching was carried out to locate the lowest energy conformer of each compound, and vibrational frequencies were computed at the same level of theory to confirm that these are indeed global minimum energy structures. Geometry optimization and frequency calculations were performed using the Gaussian09 program. ${ }^{[12]}$ The atomic coefficients of the $\mathrm{p}$ orbitals were calculated at the B3LYP/6-31G(d) level of theory and these were carried out using the GAMESSPLUS package. ${ }^{[13]}$

Table S1. The B3LYP/6-31G(d) orbital coefficients of the $2 p_{z}$ and $3 p_{z}$ atomic orbitals on carbon atoms $\mathrm{C} 2$ and $\mathrm{C} 3$ in the $\mathrm{LUMO}^{a}$ of the dienophile.

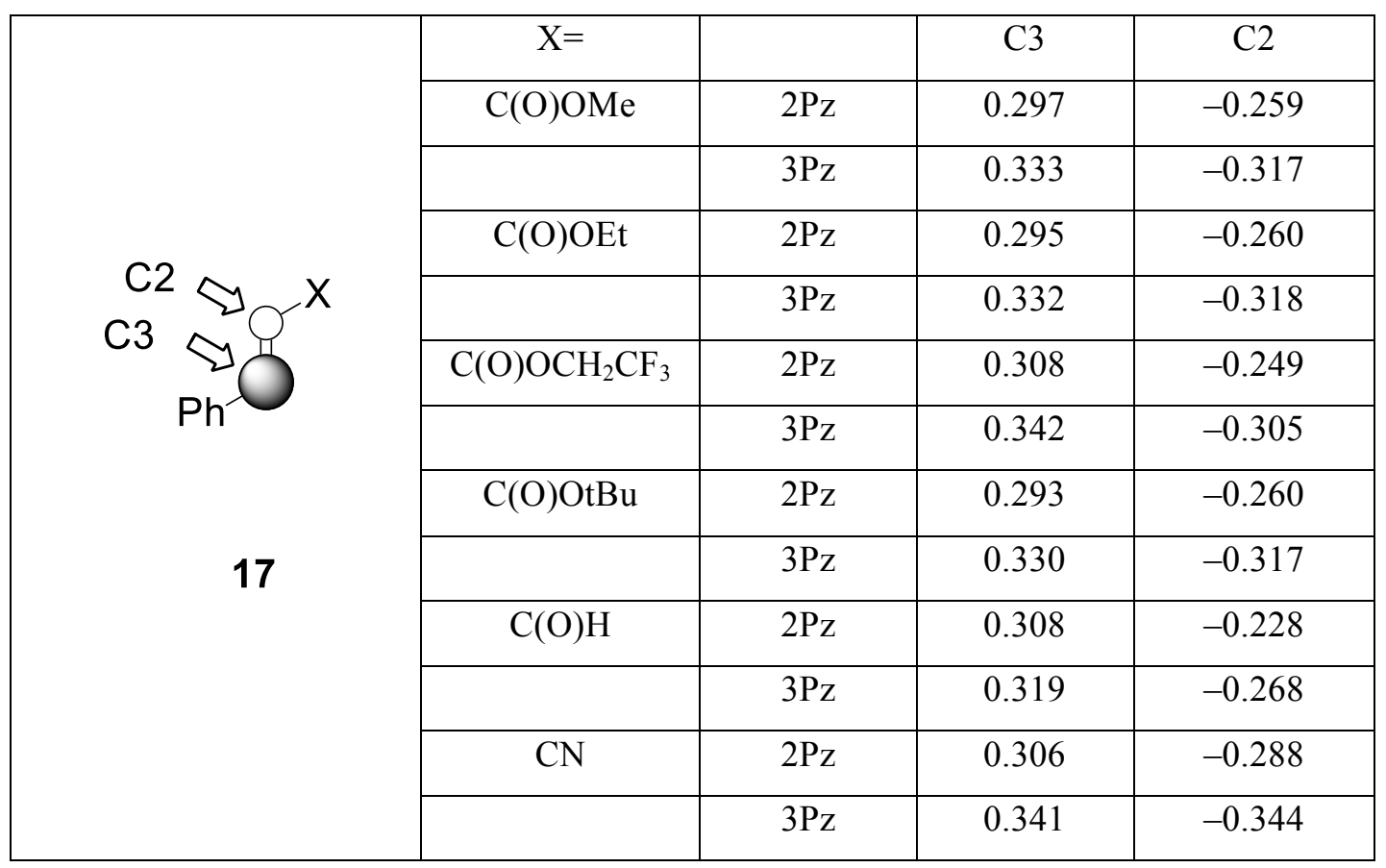

${ }^{a}$ The HOMO(diene)/LUMO(dienophile) pair has the smaller HOMO-LUMO energy gap.

\section{B3LYP/6-31+G(d) Optimized Geometries}

\section{(E)-Ocimene 8}

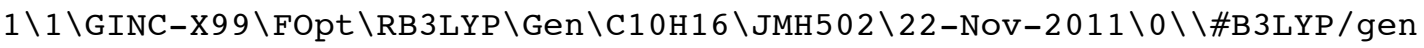

6D INT(grid=ultrafine) OPT IOP(2/17=4) Freq=noraman

maxdisk=458752000\\diene. $a 1 b 3$. freq $\backslash \backslash 0,1 \backslash C,-0.0405851764,0.0105054699,0$

$.0053161552 \backslash \mathrm{C},-0.0442948282,0.1664628127,1.3377679647 \backslash \mathrm{H}, 0.9215610749$,- 
$0.1183696032,-0.4959264884 \backslash \mathrm{H}, 0.8835605129,0.2155148495,1.9017734026 \backslash \mathrm{H}$, $-0.9689966901,0.2416491764,1.9055077407 \backslash \mathrm{C},-1.2287195827,-0.0503967268$, $-0.8766232606 \backslash \mathrm{C},-2.2967446031,0.7421459353,-0.6488334183 \backslash \mathrm{C},-1.10425571$ $88,-1.0309566721,-2.0233016309 \backslash \mathrm{C},-3.60857321,0.7953604735,-1.397199929$ $9 \backslash \mathrm{H},-2.2330346664,1.437434396,0.1884548776 \backslash \mathrm{H},-0.2038744121,-0.81592322$ $2,-2.6154786821 \backslash \mathrm{H},-0.9951238907,-2.0577121945,-1.6481110559 \backslash \mathrm{H},-1.96019$ $63824,-1.0105401052,-2.7018793014 \backslash \mathrm{H},-3.7676063685,1.8162141509,-1.7659$ $833268 \backslash \mathrm{H},-3.5774946959,0.1492196623,-2.2828318228 \backslash \mathrm{C},-4.761283379,0.363$ $427998,-0.5158688005 \backslash \mathrm{C},-5.8030781834,1.0927884071,-0.0786041533 \backslash \mathrm{H},-4.7$ $053029023,-0.6777392625,-0.1930509066 \backslash C,-6.8581639933,0.4729943067,0.8$ $099671274 \backslash \mathrm{C},-6.0440187583,2.5479564662,-0.4045343435 \backslash \mathrm{H},-7.0077173851,2$ $.6744489057,-0.9184022331 \backslash \mathrm{H},-6.1031850757,3.144183093,0.5172299229 \backslash \mathrm{H},-$ $5.2685482369,2.9877962368,-1.0364832721 \backslash \mathrm{H},-7.852657732,0.5354209961,0$. $3449275467 \backslash \mathrm{H},-6.6477189415,-0.5807292124,1.0209930472 \backslash \mathrm{H},-6.9284141122$, $1.0053285449,1.7696192395 \backslash \backslash$ Version=EM64L-G03RevE. $01 \backslash$ State=1-A $\backslash H F=-390$. $662807 \backslash \mathrm{RMSD}=4.610 \mathrm{e}-09 \backslash \mathrm{RMSF}=3.957 \mathrm{e}-06 \backslash \mathrm{Thermal}=0 . \backslash \mathrm{Dipole}=-0.1884948,0.04$ $73043,-0.1222158 \backslash \mathrm{PG}=\mathrm{C} 01 \quad[\mathrm{X}(\mathrm{C} 10 \mathrm{H} 16)] \backslash \backslash @$

\section{7, $\mathrm{X}=\mathrm{CN}$}

$1 \backslash 1 \backslash G I N C-X 99 \backslash P O p t \backslash R B 3 L Y P \backslash G e n \backslash C 9 H 7 N 1 \backslash J M H 502 \backslash 16-N o v-2011 \backslash 1 \backslash \backslash$ \#B3LYP/gen 6D INT(grid=ultrafine) OPT=(z-matrix) IOP(2/17=4) Freq=noraman maxdisk=458752000 \eto-dienophile.freq $\backslash \backslash 0,1 \backslash \mathrm{C} \backslash \mathrm{C}, 1, \mathrm{cc} 2 \backslash \mathrm{C}, 2, \mathrm{cc} 3,1, \mathrm{ccc} 3 \backslash \mathrm{C}$ $, 3, \operatorname{cc} 4,2, \operatorname{ccc} 4,1, \operatorname{dih} 4,0 \backslash \mathrm{C}, 4, \operatorname{cc} 5,3, \operatorname{ccc} 5,2, \operatorname{dih} 5,0 \backslash c, 1, \operatorname{cc} 6,2, \operatorname{ccc} 6,3, \operatorname{dih} 6,0$ $\backslash \mathrm{H}, 2, \mathrm{hc} 7,1, \operatorname{hcc} 7,6, \operatorname{dih} 7,0 \backslash \mathrm{H}, 3, \mathrm{hc} 8,2, \mathrm{hcc} 8,1, \operatorname{dih} 8,0 \backslash \mathrm{H}, 4, \mathrm{hc} 9,3, \mathrm{hcc} 9,2$, dih 9 $, 0 \backslash \mathrm{H}, 5, \operatorname{hc} 10,4, \operatorname{hcc} 10,3, \operatorname{dih} 10,0 \backslash \mathrm{H}, 6, \operatorname{hc} 11,1, \operatorname{hcc} 11,2, \operatorname{dih} 11,0 \backslash \mathrm{C}, 1, \operatorname{cc} 12,2, \mathrm{Cc}$ $\mathrm{c} 12,3, \operatorname{dih} 12,0 \backslash \mathrm{C}, 12, \operatorname{cc} 13,1, \operatorname{ccc} 13,2, \operatorname{dih} 13,0 \backslash \mathrm{H}, 12, \operatorname{hc} 14,1, \operatorname{hcc} 14,2, \operatorname{dih} 14,0 \backslash$ $\mathrm{C}, 13, \operatorname{cc} 15,12, \operatorname{ccc} 15,1, \operatorname{dih} 15,0 \backslash \mathrm{H}, 13, \operatorname{hc} 16,12, \operatorname{hcc} 16,1, \operatorname{dih} 16,0 \backslash x, 15,1 ., 13,9$ $0 ., 12,180 ., 0 \backslash \mathrm{N}, 15, \mathrm{nc} 18,17, \operatorname{ncxx} 18,13, \operatorname{dih} 18,0 \backslash \backslash \mathrm{cc} 2=1.40972775 \backslash \mathrm{cc} 3=1.3920$ $2365 \backslash \operatorname{ccc} 3=120.69522174 \backslash \operatorname{cc} 4=1.40078177 \backslash \operatorname{ccc} 4=120.34102277 \backslash \mathrm{dih} 4=0 . \backslash \operatorname{cc} 5=1$. $39668263 \backslash \operatorname{ccc} 5=119.68737746 \backslash \mathrm{dih} 5=0 . \backslash \mathrm{cc} 6=1.40809063 \backslash \mathrm{CCC} 6=118.25289198 \backslash \mathrm{di}$ h $6=0 . \backslash$ hc $7=1.08622795 \backslash$ hCc $7=120.19034854 \backslash \mathrm{dih} 7=180 . \backslash$ hc $8=1.08678972 \backslash \mathrm{hcc} 8=1$ $19.65619899 \backslash \mathrm{dih} 8=180 . \backslash \mathrm{hc} 9=1.08671384 \backslash \mathrm{hcc} 9=120.09408092 \backslash \mathrm{dih} 9=180 . \backslash \mathrm{hc} 10=$ $1.08668184 \backslash \operatorname{hcc} 10=120.23013944 \backslash$ dih $10=180 . \backslash$ hc $11=1.08770529 \backslash$ hcc $11=119.213$ $57822 \backslash \mathrm{dih} 11=180 . \backslash \mathrm{cc} 12=1.46394125 \backslash \mathrm{ccc} 12=123.38454357 \backslash \mathrm{dih} 12=180 . \backslash \operatorname{cc} 13=1$. $35207371 \backslash \operatorname{ccc} 13=127.1302215 \backslash \operatorname{dih} 13=0 . \backslash \mathrm{hc} 14=1.08889079 \backslash \mathrm{hcc} 14=115.31291014$ $\backslash \mathrm{dih} 14=180 . \backslash \mathrm{CC} 15=1.42528951 \backslash \mathrm{CCC} 15=122.12993471 \backslash \mathrm{dih} 15=180 . \backslash \mathrm{hc} 16=1.08690$ $591 \backslash \mathrm{hcc} 16=122.2071591 \backslash \mathrm{dih} 16=0 . \backslash \mathrm{nc} 18=1.16567148 \backslash \mathrm{ncxx} 18=88.83624971 \backslash \mathrm{dih} 1$

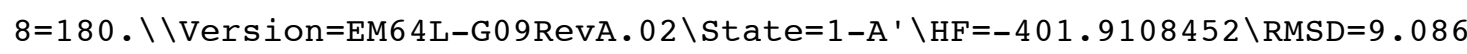
e-09 $\backslash$ RMSF $=2.509 e-05 \backslash \mathrm{Dipole}=1.9149371,0 ., 1.0754856 \backslash$ Quadrupole=-6.466058 $6,1.1711651,5.2948935,0 .,-9.1619617,0 . \backslash P G=C S \quad[S G(C 9 H 7 N 1)] \backslash \backslash @$ 


\section{7, $\mathrm{X}=\mathrm{CHO}$}

$1 \backslash 1 \backslash G I N C-X 148 \backslash F O p t \backslash R B 3 L Y P \backslash G e n \backslash C 9 H 8 O 1 \backslash J M H 502 \backslash 15-N o v-2011 \backslash 0 \backslash \backslash \# B 3 L Y P / g e n$ 6D INT(grid=ultrafine) OPT IOP $(2 / 17=4)$ Freq=noraman

maxdisk $=458752000 \backslash \backslash$ formyl-dienophile.freq $\backslash \backslash 0,1 \backslash C,-0.0167218863,0,0.11$ $30606826 \backslash \mathrm{C}, 0.0938643813,0 ., 1.5187258546 \backslash \mathrm{C}, 1.3431657735,0 ., 2.1329476005$ $\backslash C, 2.511401109,0,, 1.3600981269 \backslash \mathrm{C}, 2.4197895056,0,-0.0339656025 \backslash \mathrm{C}, 1.167$ $7659125,0 .,-0.6490286164 \backslash \mathrm{H},-0.8014935685,0 ., 2.1333144299 \backslash \mathrm{H}, 1.409319515$ $7,0 ., 3.217746443 \backslash \mathrm{H}, 3.4846411761,0 ., 1.8437423773 \backslash \mathrm{H}, 3.3210379443,0 .,-0.6$ $412202033 \backslash \mathrm{H}, 1.1001538185,0 .,-1.7347988936 \backslash \mathrm{C},-1.3025437611,0 .,-0.585033$ $8796 \backslash \mathrm{C},-2.544757744,0 .,-0.0509745077 \backslash \mathrm{H},-1.2315654148,0,,-1.6746930639 \backslash$ $\mathrm{C},-3.7273303853,0 .,-0.9135582026 \backslash \mathrm{H},-2.7315685261,0,1.0201983024 \backslash \mathrm{O},-4$. $8798497167,0 .,-0.5081965743 \backslash \mathrm{H},-3.5176191337,0 .,-2.0063642731 \backslash \backslash$ Version= EM64L-G03RevE. $01 \backslash$ State $=1-A^{\prime} \backslash \mathrm{HF}=-422.9953337 \backslash \mathrm{RMSD}=9.955 \mathrm{e}-09 \backslash \mathrm{RMSF}=1.935 \mathrm{e}$ $-05 \backslash$ Thermal $=0 . \backslash$ Dipole $=1.9675194,0 ., 0.0161028 \backslash P G=C S \quad[S G(C 9 H 801)] \backslash \backslash @$

\section{$17, \mathrm{X}=\mathrm{COOMe}$}

$1 \backslash 1 \backslash G I N C-V 1257 \backslash F O p t \backslash R B 3 L Y P \backslash G e n \backslash C 10 H 1002 \backslash J M H 502 \backslash 22-O C t-2012 \backslash 0 \backslash \backslash \# B 3 L Y P / g$ en 6D INT(grid=ultrafine) OPT IOP(2/17=4) Freq=noraman maxdisk $=262144000 \backslash \backslash$ meo-dienophile.a2 .freq $\backslash \backslash 0,1 \backslash C,-0.0568095831,0 ., 0.15$ $39806611 \backslash C, 0.1192030325,0,1.5526533555 \backslash C, 1.3953096355,0 ., 2.1095798187$ $\backslash C, 2.5274114944,0 ., 1.2846284521 \backslash \mathrm{C}, 2.3711524675,0,,-0.1034466023 \backslash \mathrm{C}, 1.09$ $17580884,0,-0.6606262285 \backslash \mathrm{H},-0.7466318282,0 ., 2.2085510619 \backslash \mathrm{H}, 1.51072631$ $07,0 ., 3.1904279658 \backslash \mathrm{H}, 3.5217725036,0$, $1.7234239674 \backslash \mathrm{H}, 3.2432761814,0$,, 0 $.7520375516 \backslash \mathrm{H}, 0.9733917023,0 .,-1.7418507797 \backslash \mathrm{C},-1.371778403,0 .,-0.49149$ $98435 \backslash \mathrm{C},-2.5816600072,0 ., 0.1023383845 \backslash \mathrm{H},-1.3646056408,0,,-1.5810972077$ $\backslash \mathrm{C},-3.8130148347,0,,-0.7112101162 \backslash \mathrm{H},-2.7136941481,0 ., 1.1796187731 \backslash \mathrm{O},-3$ $.8732136702,0 .,-1.9291851961 \backslash 0,-4.9138700359,0 ., 0.0833950251 \backslash \mathrm{C},-6.1790$ $798991,0,-0.5992415626 \backslash \mathrm{H},-6.9324328767,0 ., 0.1891723536 \backslash \mathrm{H},-6.274260244$ $7,0.8912779056,-1.2257008652 \backslash \mathrm{H},-6.2742602447,-0.8912779056,-1.22570086$ $52 \backslash \backslash$ Version=EM64L-G09RevA. 02 $\backslash$ State $=1-A^{\prime} \backslash \mathrm{HF}=-537.5534476 \backslash \mathrm{RMSD}=6.687 \mathrm{e}-09$ $\backslash \mathrm{RMSF}=8.357 \mathrm{e}-05 \backslash \mathrm{Dipole}=0.4094065,0 ., 0.7496465 \backslash$ Quadrupole $=9.1843168,-5$. $7494471,-3.4348697,0 .,-1.7454724,0 . \backslash P G=C S \quad[S G(\mathrm{C} 10 \mathrm{H} 802), x(\mathrm{H} 2)] \backslash \backslash @$

\section{7, $\mathrm{X}=$ COOEt}

$1 \backslash 1 \backslash G I N C-V 1273 \backslash F O p t \backslash R B 3 L Y P \backslash G e n \backslash C 11 H 12 O 2 \backslash J M H 502 \backslash 22-O C t-2012 \backslash 0 \backslash \backslash \# B 3 L Y P / g$ en 6D INT(grid=ultrafine) OPT IOP(2/17=4) Freq=noraman maxdisk=262144000 \eto-dienophile.a2.global.freq $\backslash \backslash 0,1 \backslash \mathrm{C},-0.10162945,0$. $, 0.1418276683 \backslash \mathrm{C}, 0.1123673626,0 ., 1.5352812671 \backslash \mathrm{C}, 1.4030566227,0 ., 2.05758$ $31085 \backslash \mathrm{C}, 2.512459711,0 ., 1.2023454413 \backslash \mathrm{C}, 2.3185726265,0 .,-0.1809455986 \backslash \mathrm{C}$, $1.0245575452,0 .,-0.7033808495 \backslash \mathrm{H},-0.7354934204,0 ., 2.2142095494 \backslash \mathrm{H}, 1.5476$ $95064,0 ., 3.1349240724 \backslash \mathrm{H}, 3.5183806145,0 ., 1.6139833654 \backslash \mathrm{H}, 3.172849673,0 .$, $-0.8528990589 \backslash \mathrm{H}, 0.8769739952,0,,-1.7810106509 \backslash \mathrm{C},-1.4338151997,0,-0.46$ $76491127 \backslash \mathrm{C},-2.626947913,0 ., 0.1585689676 \backslash \mathrm{H},-1.456834658,0 .,-1.557047015$ 
$6 \backslash \mathrm{C},-3.8817531331,0 .,-0.6203529658 \backslash \mathrm{H},-2.7297977777,0.1 .2392376423 \backslash 0,-$ $3.9719675127,0 \ldots,-1.8367933122 \backslash 0,-4.9589682294,0 ., 0.2046997777 \backslash \mathrm{C},-6.257$ $5732826,0 .,-0.4337458771 \backslash \mathrm{C},-7.3082361785,0.0 .661340653 \backslash \mathrm{H},-6.330960470$ $4,0.8846591957,-1.074865295 \backslash \mathrm{H},-6.3309604704,-0.8846591957,-1.074865295$ $\backslash \mathrm{H},-8.3084083308,0 ., 0.2122415983 \backslash \mathrm{H},-7.2160995941,-0.8884785981,1.29542$ $04599 \backslash \mathrm{H},-7.2160995941,0.8884785981,1.2954204599 \backslash \backslash$ Version=EM64L-G09RevA $.02 \backslash$ State $=1-A^{\prime} \backslash H F=-576.8731192 \backslash \mathrm{RMSD}=5.931 \mathrm{e}-09 \backslash \mathrm{RMSF}=1.659 \mathrm{e}-05 \backslash \mathrm{Dipole}=0$. $3270667,0.0 .7837774 \backslash$ Quadrupole $=10.3219293,-6.1433115,-4.1786178,0 .,-1$ $.7183739,0 . \backslash \mathrm{PG}=\mathrm{CS}[\mathrm{SG}(\mathrm{C} 11 \mathrm{H} 802), \mathrm{X}(\mathrm{H} 4)] \backslash \backslash @$

\section{7, $\mathrm{X}=\mathrm{COOCH}_{2} \mathrm{CF}_{3}$}

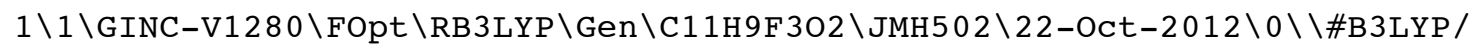
gen 6D INT(grid=ultrafine) OPT IOP(2/17=4) Freq=noraman

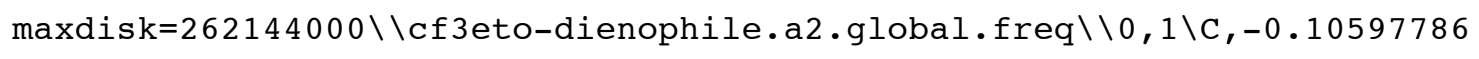
$44,0.0 .1489573233 \backslash \mathrm{C}, 0.1164207957,0$. $1.5413073614 \backslash \mathrm{C}, 1.410658703,0.2 .0$ $538142773 \backslash \mathrm{C}, 2.5134159649,0$. $1.1897886152 \backslash \mathrm{C}, 2.3108191346,0$. -0.19231898 $41 \backslash \mathrm{C}, 1.0134491039,0 .,-0.7055916011 \backslash \mathrm{H},-0.7264007274,0 ., 2.2263528581 \backslash \mathrm{H}, 1$

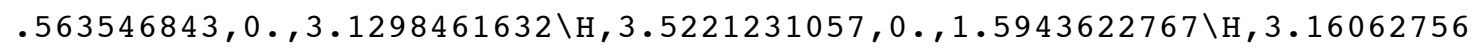
$62,0 .,-0.8696609589 \backslash \mathrm{H}, 0.8580456679,0,-1.7820560709 \backslash \mathrm{C},-1.4406034612,0$. $,-0.4504547748 \backslash \mathrm{C},-2.6303438756,0$. $0.1853491083 \backslash \mathrm{H},-1.4703300956,0$.,-1.5 $396642578 \backslash \mathrm{C},-3.8813592685,0,-0.5866170378 \backslash \mathrm{H},-2.7286755958,0.1 .265964$ $872 \backslash 0,-4.0007026814,0 \ldots,-1.797899421 \backslash 0,-4.963587941,0.0 .252082391 \backslash \mathrm{C},-6$ $.2319028487,0 .,-0.3937687673 \backslash \mathrm{C},-7.2935729678,0.0 .6915448765 \backslash \mathrm{H},-6.3566$ $719549,0.8903775336,-1.0166196854 \backslash \mathrm{H},-6.3566719549,-0.8903775336,-1.016$ $6196854 \backslash \mathrm{F},-8.5203008794,0.0 .1115912822 \backslash \mathrm{F},-7.2182773841,-1.0897798076$, $1.4857709192 \backslash \mathrm{F},-7.2182773841,1.0897798076,1.4857709192 \backslash \backslash$ Version=EM64LG09RevA. 02 \State $=1-A^{\prime} \backslash H F=-874.6121058 \backslash R M S D=5.655 e-09 \backslash R M S F=1.100 e-05 \backslash D i$ pole $=1.5236707,0.0 .167744 \backslash$ Quadrupole $=5.1911122,-3.823625,-1.3674873,0$ $., 3.7577159,0 . \backslash \mathrm{PG}=\mathrm{CS}[\mathrm{SG}(\mathrm{C} 11 \mathrm{H} 7 \mathrm{~F} 1 \mathrm{O} 2), \mathrm{X}(\mathrm{H} 2 \mathrm{~F} 2)] \backslash \backslash \mathrm{a}$

\section{$17, \mathrm{X}=\mathrm{COO}^{t} \mathrm{Bu}$}

$1 \backslash 1 \backslash G I N C-V 1292 \backslash F O p t \backslash R B 3 L Y P \backslash G e n \backslash C 13 H 1602 \backslash J M H 502 \backslash 22-O c t-2012 \backslash 0 \backslash \backslash \# B 3 L Y P / g$ en 6D INT(grid=ultrafine) OPT IOP(2/17=4) Freq=noraman maxdisk=262144000\\tbuo-dienophile.a2.global.freq $\backslash \backslash 0,1 \backslash \mathrm{C},-0.0716381767$ $, 0.0 .1672657784 \backslash \mathrm{C}, 0.2132377672,0.1 .5479482657 \backslash \mathrm{C}, 1.5289158178,0.2 .00$ $39001579 \backslash \mathrm{C}, 2.5935066454,0$. $1.0935077289 \backslash \mathrm{C}, 2.3292835523,0$, -0.278102396 $8 \backslash \mathrm{C}, 1.0102354193,0 \ldots,-0.7339350536 \backslash \mathrm{H},-0.5991511736,0$. 2.2689305607\H, 1 .

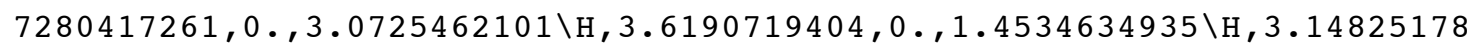
$85,0 .,-0.9927012516 \backslash \mathrm{H}, 0.8079992652,0,-1.8026542665 \backslash \mathrm{C},-1.4335726932,0$. $,-0.373390542 \backslash \mathrm{C},-2.5928527927,0.0 .3128328381 \backslash \mathrm{H},-1.5126977181,0 .,-1.46$ $01549801 \backslash \mathrm{C},-3.8894951393,0 .,-0.4027349285 \backslash \mathrm{H},-2.639326372,0 ., 1.39731322$ $64 \backslash 0,-4.0207626394,0 .,-1.6161881662 \backslash 0,-4.9080658414,0 \ldots, 0.4889704209 \backslash \mathrm{C}$, $-6.3237417647,0.0 .0674105871 \backslash \mathrm{C},-7.0698069704,0 ., 1.4048128974 \backslash \mathrm{C},-6.635$ 
$0340177,1.2737831142,-0.7268846685 \backslash \mathrm{C},-6.6350340177,-1.2737831142,-0.72$ $68846685 \backslash \mathrm{H},-8.1516494195,0$. $1.2309046327 \backslash \mathrm{H},-6.8130565019,-0.8881935582$ $, 1.9922416457 \backslash \mathrm{H},-6.8130565019,0.8881935582,1.9922416457 \backslash \mathrm{H},-7.713825392$ $9,1.3288702352,-0.9156599551 \backslash \mathrm{H},-6.3457494252,2.162140432,-0.1536831871$ $\backslash \mathrm{H},-6.1123977731,1.2858983222,-1.6852839419 \backslash \mathrm{H},-7.7138253929,-1.3288702$ $352,-0.9156599551 \backslash \mathrm{H},-6.1123977731,-1.2858983222,-1.6852839419 \backslash \mathrm{H},-6.345$ $7494252,-2.162140432,-0.1536831871 \backslash \backslash$ Version=EM64L-G09RevA.02 $\backslash$ State=1-A ' $\backslash \mathrm{HF}=-655.5049961 \backslash \mathrm{RMSD}=1.892 \mathrm{e}-09 \backslash \mathrm{RMSF}=1.197 \mathrm{e}-05 \backslash \mathrm{Dipole}=0.2483293,0 ., 0$. $7014221 \backslash$ Quadrupole $=10.4770191,-6.7467768,-3.7302423,0 .,-1.009475,0 . \backslash P G$ $=\mathrm{CS}[\mathrm{SG}(\mathrm{C} 11 \mathrm{H} 8 \mathrm{O} 2), \mathrm{X}(\mathrm{C} 2 \mathrm{H} 8)] \backslash \backslash \mathrm{Q}$

\section{Binding Studies by NMR Spectroscopy}

The construct of the dengue virus NS2B-NS3 protease used in this work was described previously. ${ }^{[14]}$ The protein was expressed in vivo in the E. coli strain Rosetta:: $\lambda$ DE3/pRARE using a high-cell density method. ${ }^{[15]}$ The pelleted cells were re-suspended in $50 \mathrm{~mL}$ lysis buffer A (50 mM HEPES, pH 7.5, $300 \mathrm{mM} \mathrm{NaCl}$ ), disrupted by French press lysis at 12000 psi and centrifuged at $16000 \times \mathrm{g}$ for $45 \mathrm{~min}$. at $4^{\circ} \mathrm{C}$ to remove the cell debris. The supernatant was filtered (Sartorius $0.45 \mu \mathrm{m}$ filter) and loaded onto a 5-mL HisTrap HP column (GE Healthcare) equilibrated with buffer A. After washing the column with $20 \mathrm{~mL}$ buffer $A$ at a flow rate of $1 \mathrm{~mL} / \mathrm{min}$, a gradient from 15-500 mM imidazole was applied over $60 \mathrm{~min}$. The dengue virus protease eluted at $200 \mathrm{mM}$ imidazole. Fractions containing the protease were pooled, dialyzed against NMR buffer (20 mM Tris, $\mathrm{pH}$ 6.9, $50 \mathrm{mM} \mathrm{NaCl}$ ) and loaded onto a $50 \mathrm{~mL}$ Toyopearl DEAE-650M column equilibrated with the same buffer. After washing the column with $50 \mathrm{~mL}$ of NMR buffer, a gradient from 50 $\mathrm{mM}$ to $1 \mathrm{M} \mathrm{NaCl}$ was applied over $200 \mathrm{~mL}$. The dengue virus protease eluted at $300 \mathrm{mM} \mathrm{NaCl}$. Fractions containing the dengue virus protease were pooled, dialyzed against NMR buffer and concentrated in a centrifugal filter device (Millipore, MWCO 10000) for NMR experiments.

All NMR spectra were recorded at $25^{\circ} \mathrm{C}$ on a Bruker $800 \mathrm{MHz}$ NMR spectrometer equipped with a cryoprobe. $0.1 \mathrm{mM}$ samples of the dengue virus protease were prepared in NMR buffer $(20 \mathrm{mM}$ Tris, $\mathrm{pH}$ 6.9, $50 \mathrm{mM} \mathrm{NaCl}$ ). In different experiments, panduratin A 1 and 4-hydroxypanduratin A 2 were individually added in equimolar ratio and in five-fold molar excess to the protein from a 100 $\mathrm{mM}$ stock solution in DMSO- $\mathrm{d}_{6}$. 


\section{References}

[1] W. L. F. Armarego, C. L. L. Chai, Purification of Laboratory Chemicals, 5th ed., Butterworth-Heinemann, Burlington, MA, 2003.

[2] T.-S. Chou, H.-H. Tso, L.-J. Chang, J. Chem. Soc., Chem. Commun. 1984, 1323-1324.

[3] N. N. Win, S. Awale, H. Esumi, Y. Tezuka, S. Kadota, Chem. Pharm. Bull. 2008, 56, 491496.

[4] B. M. Trost, F. D. Toste, J. Am. Chem. Soc. 2003, 125, 3090-3100.

[5] E. Kiehlmann, R. W. Lauener, Can. J. Chem. 1989, 67, 335-344.

[6] P. Bañuelos, J. M. García, E. Gómez-Bengoa, A. Herrero, J. M. Odriozola, M. Oiarbide, C. Palomo, J. Razkin, J. Org. Chem. 2010, 75, 1458-1473.

[7] K. C. Nicolaou, H. Xu, M. Wartmann, Angew. Chem. 2005, 117, 766-771; Angew. Chem. Int. Ed. 2005, 44, 756-761.

[8] P. Tuntiwachwuttikul, O. Pancharoen, V. Reutrakul, L. T. Byrne, Aust. J. Chem. 1984, 37, 449-453.

[9] G. Trakoontivakorn, K. Nakahara, H. Shinmoto, M. Takenaka, M. Onishi-Kameyama, H. Ono, M. Yoshida, T. Nagata, T. Tsushida, J. Agric. Food Chem. 2001, 49, 3046-3050.

[10] J.-Q. Gu, E. J. Park, J. S. Vigo, J. G. Graham, H. H. S. Fong, J. M. Pezzuto, A. D. Kinghorn, J. Nat. Prod. 2002, 65, 1616-1620.

[11] N. N. Win, S. Awale, H. Esumi, Y. Tezuka, S. Kadota, J. Nat. Prod. 2007, 70, 1582-1587.

[12] M. J. Frisch, G. W. Trucks, H. B. Schlegel, G. E. Scuseria, M. A. Robb, J. R. Cheeseman, G. Scalmani, V. Barone, B. Mennucci, G. A. Petersson, H. Nakatsuji, M. Caricato, X. Li, H. P. Hratchian, A. F. Izmaylov, J. Bloino, G. Zheng, J. L. Sonnenberg, M. Hada, M. Ehara, K. Toyota, R. Fukuda, J. Hasegawa, M. Ishida, T. Nakajima, Y. Honda, O. Kitao, H. Nakai, T. Vreven, J. A. Montgomery, Jr., J. E. Peralta, F. Ogliaro, M. Bearpark, J. J. Heyd, E. Brothers, K. N. Kudin, V. N. Staroverov, R. Kobayashi, J. Normand, K. Raghavachari, A. Rendell, J. C. Burant, S. S. Iyengar, J. Tomasi, M. Cossi, N. Rega, J. M. Millam, M. Klene, J. E. Knox, J. B. Cross, V. Bakken, C. Adamo, J. Jaramillo, R. Gomperts, R. E. Stratmann, O. Yazyev, A. J. Austin, R. Cammi, C. Pomelli, J. W. Ochterski, R. L. Martin, K. Morokuma, V. G. Zakrzewski, G. A. Voth, P. Salvador, J. J. Dannenberg, S. Dapprich, A. D. Daniels, Ö. Farkas, J. B. Foresman, J. V. Ortiz, J. Cioslowski, D. J. Fox, Gaussian 09, Revision A.1: Gaussian, Inc. Wallingford CT, 2009.

[13] M. Higashi, A. V. Marenich, R. M. Olson, A. Chamberlin, J. Pu, C. P. Kelly, J. D. Thompson, J. D. Xidos, J. Li, T. Zhu, G. D. Hawkins, Y.-Y. Chuang, P. L. Fast, B. J. Lynch, D. A. Liotard, D. Rinaldi, J. Gao, C. J. Cramer, D. G. Truhlar, GAMESSPLUS - version 2008-2, University 
of Minnesota, 2008, based on the General Atomic and Molecular Electronic Structure System (GAMESS) as described in M. W. Schmidt, K. K. Baldridge, J. A. Boatz, S. T. Elbert, M. S. Gordon, J. H. Jensen, S. Koseki, N. Matsunaga, K. A. Nguyen, S. Su, T. L. Windus, M. Dupuis, J. A. Montgomery Jr., J. Comp. Chem. 1993, 14, 1347-1363.

[14] L. de la Cruz, T. H. D. Nguyen, K. Ozawa, J. Shin, B. Graham, T. Huber, G. Otting, J. Am. Chem. Soc. 2011, 133, 19205-19215.

[15] A. Sivashanmugam, V. Murray, C. Cui, Y. Zhang, J. Wang, Q. Li, Protein Sci. 2009, 18, 936948. 


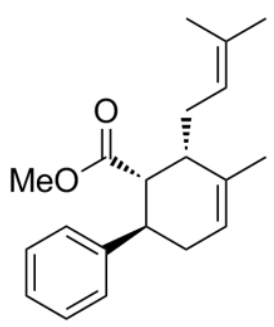

panduratin $\mathrm{H} 3$

${ }^{1} \mathrm{H}-\mathrm{NMR}$ spectrum, $400 \mathrm{MHz}, \mathrm{CDCl}_{3}$

S37 


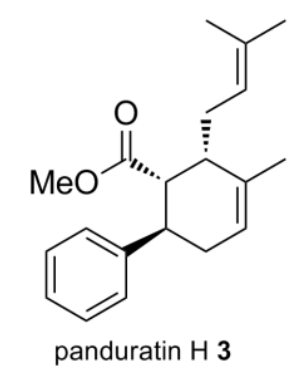

${ }^{13} \mathrm{C}$-NMR spectrum, $100 \mathrm{MHz}, \mathrm{CDCl}_{3}$

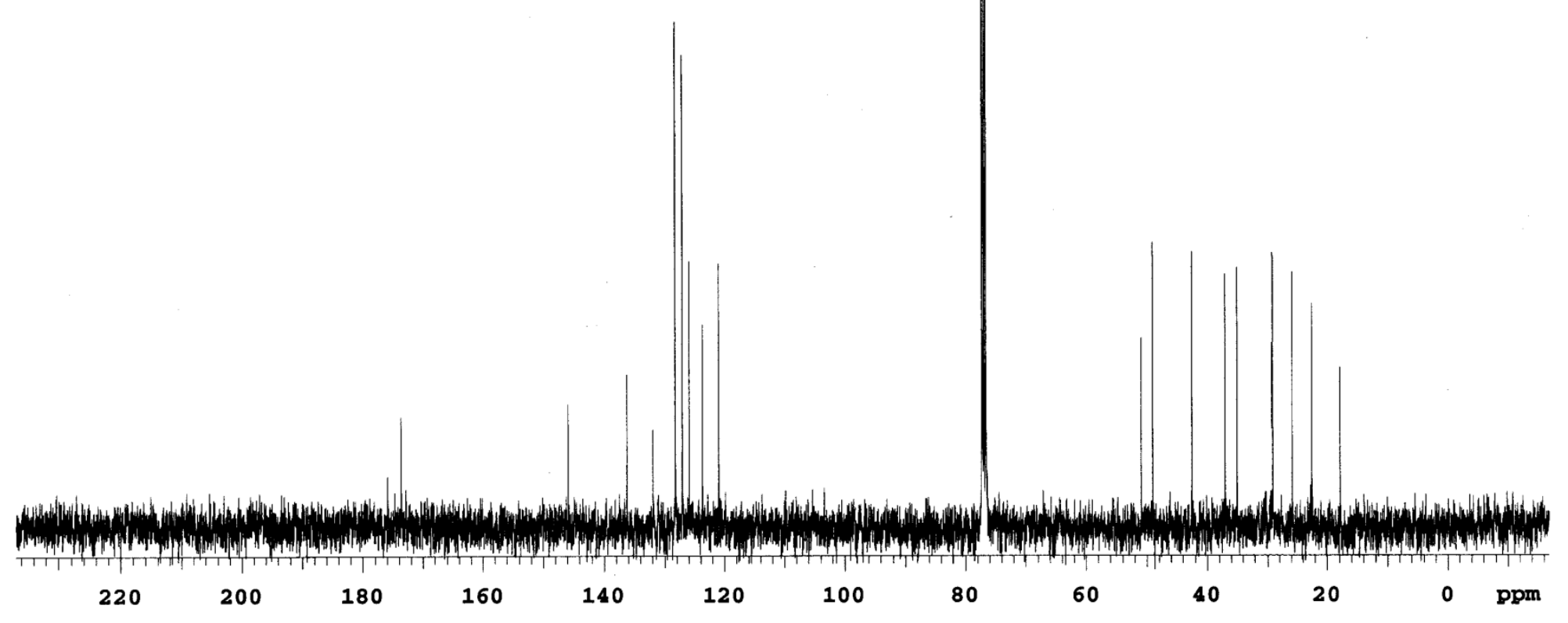




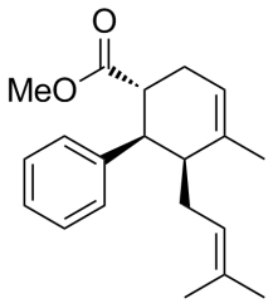

panduratin I 4

${ }^{1} \mathrm{H}-\mathrm{NMR}$ spectrum, $400 \mathrm{MHz}, \mathrm{CDCl}_{3}$

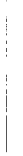

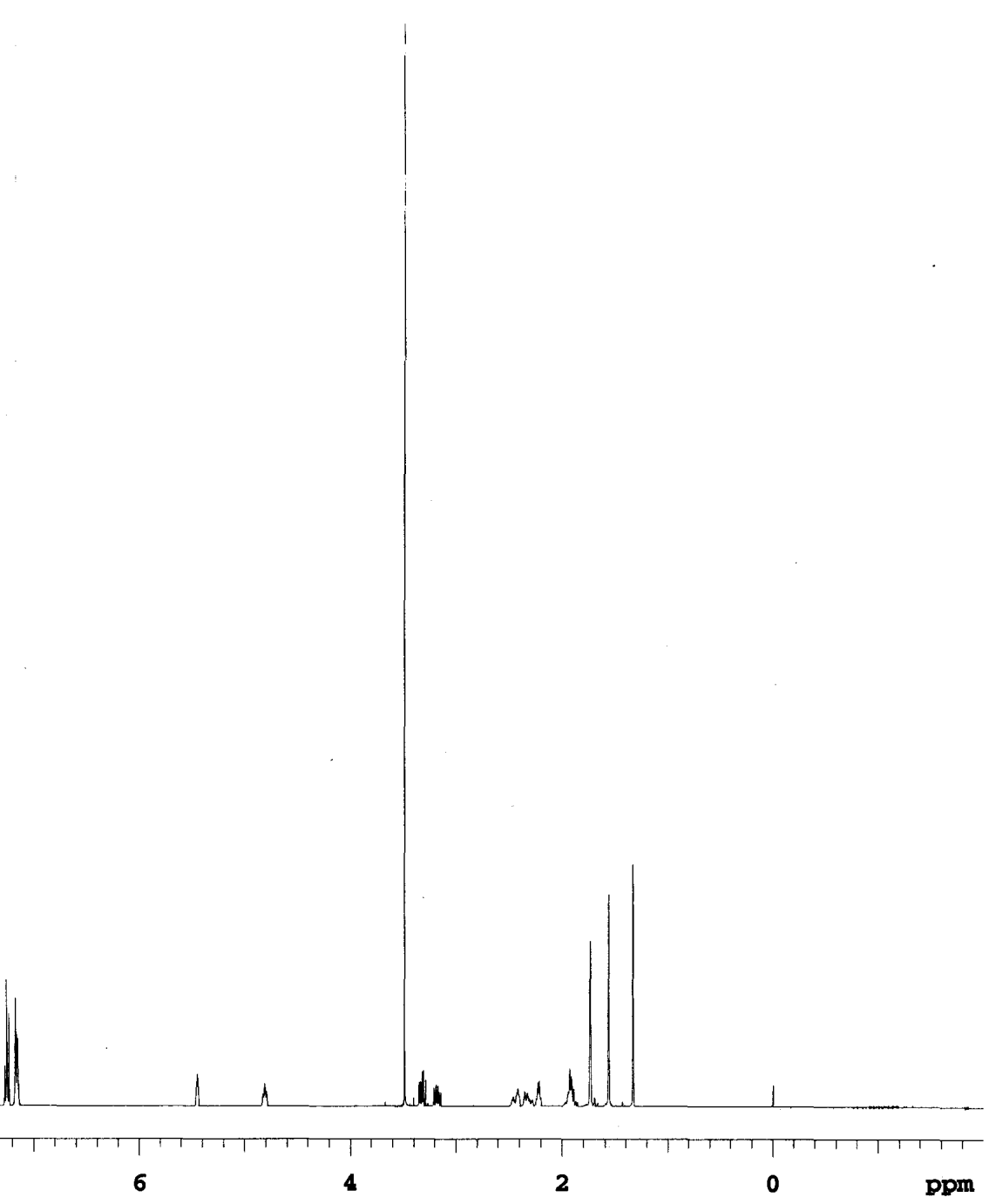

$14 \quad 12$

10

8

2

ppm

S39 


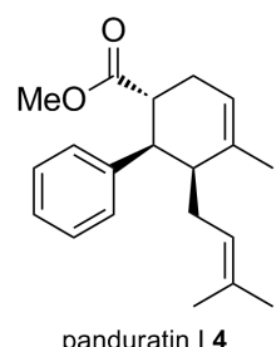

${ }^{13} \mathrm{C}-\mathrm{NMR}$ spectrum, $100 \mathrm{MHz}, \mathrm{CDCl}_{3}$

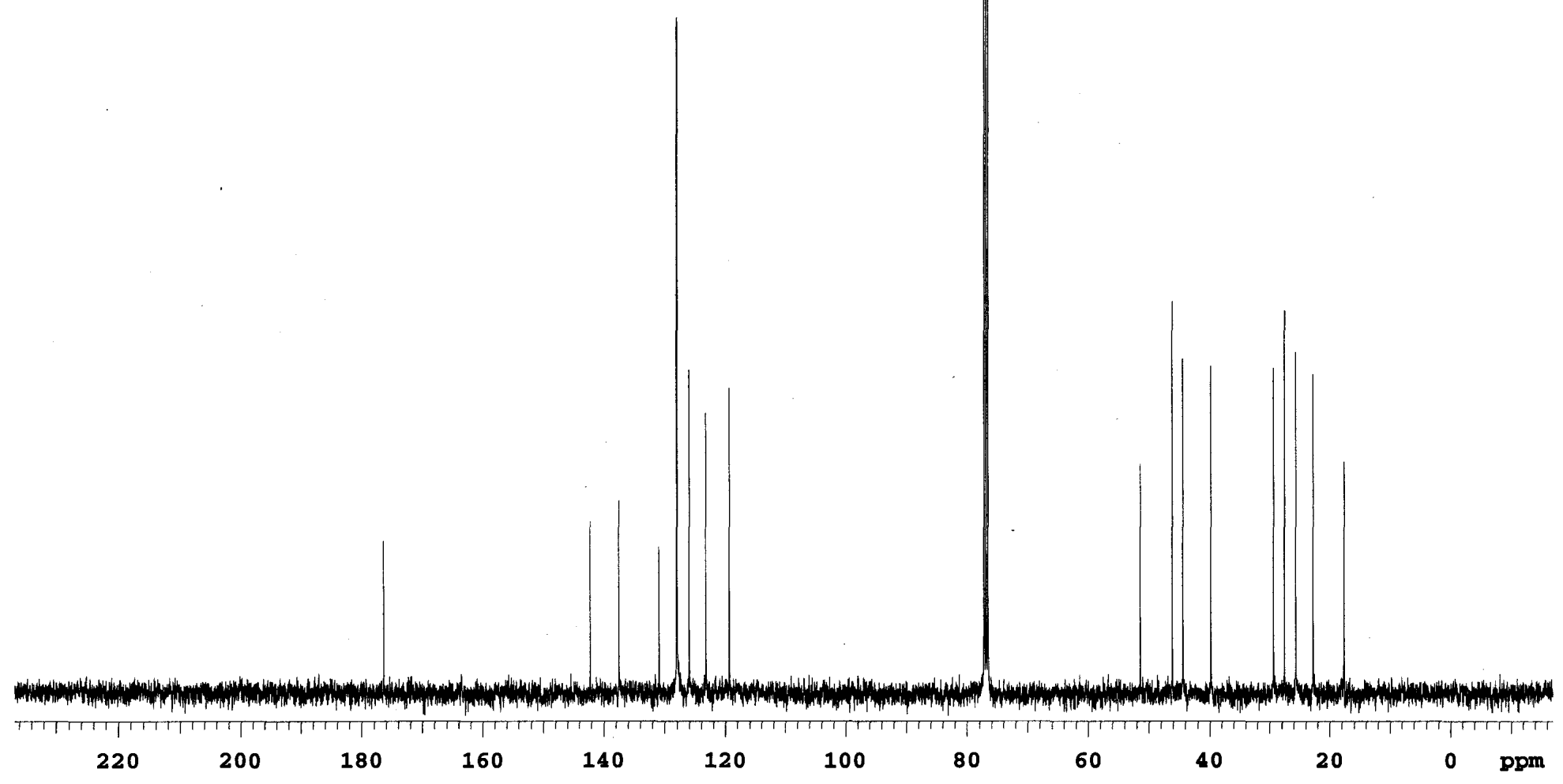




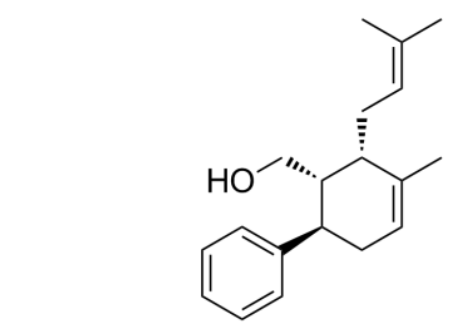

( \pm )--((1R,2S,6R)-3-methyl-2-(3-methylbut-2-en-1-yl)

-6-phenylcyclohex-3-en-1-yl)methanol 10

${ }^{1} \mathrm{H}-\mathrm{NMR}$ spectrum, $400 \mathrm{MHz} \mathrm{CDCl}_{3}$

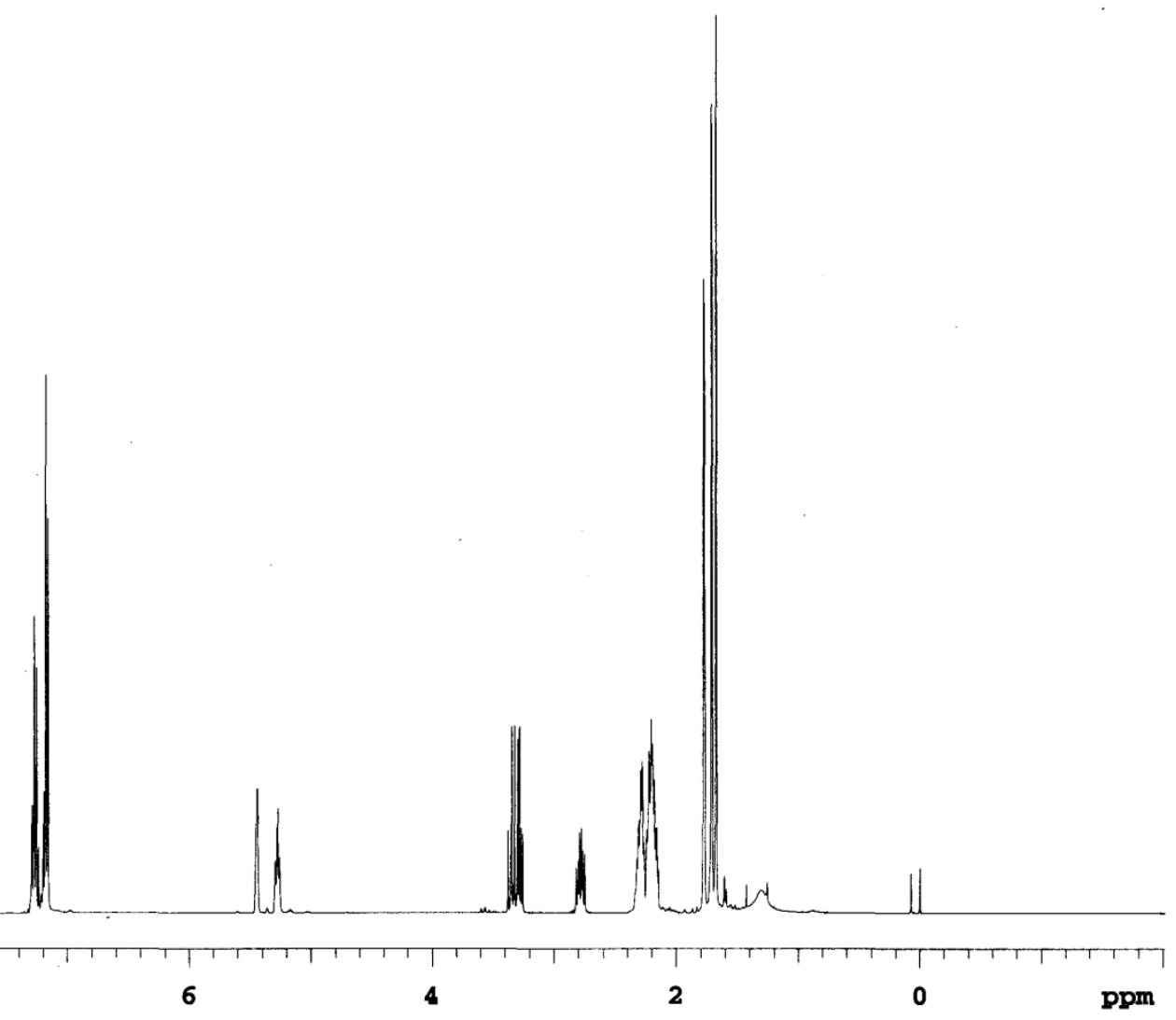




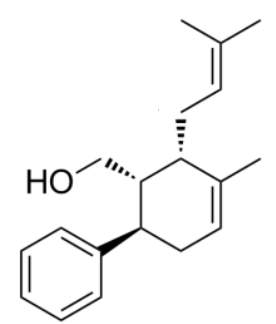

$( \pm)-((1 R, 2 S, 6 R)-3-m e t h y l-2-(3-m e t h y l b u t-2-e n-1-y l)$

-6-phenylcyclohex-3-en-1-yl)methanol 10

${ }^{13} \mathrm{C}-\mathrm{NMR}$ spectrum, $100 \mathrm{MHz}, \mathrm{CDCl}_{3}$

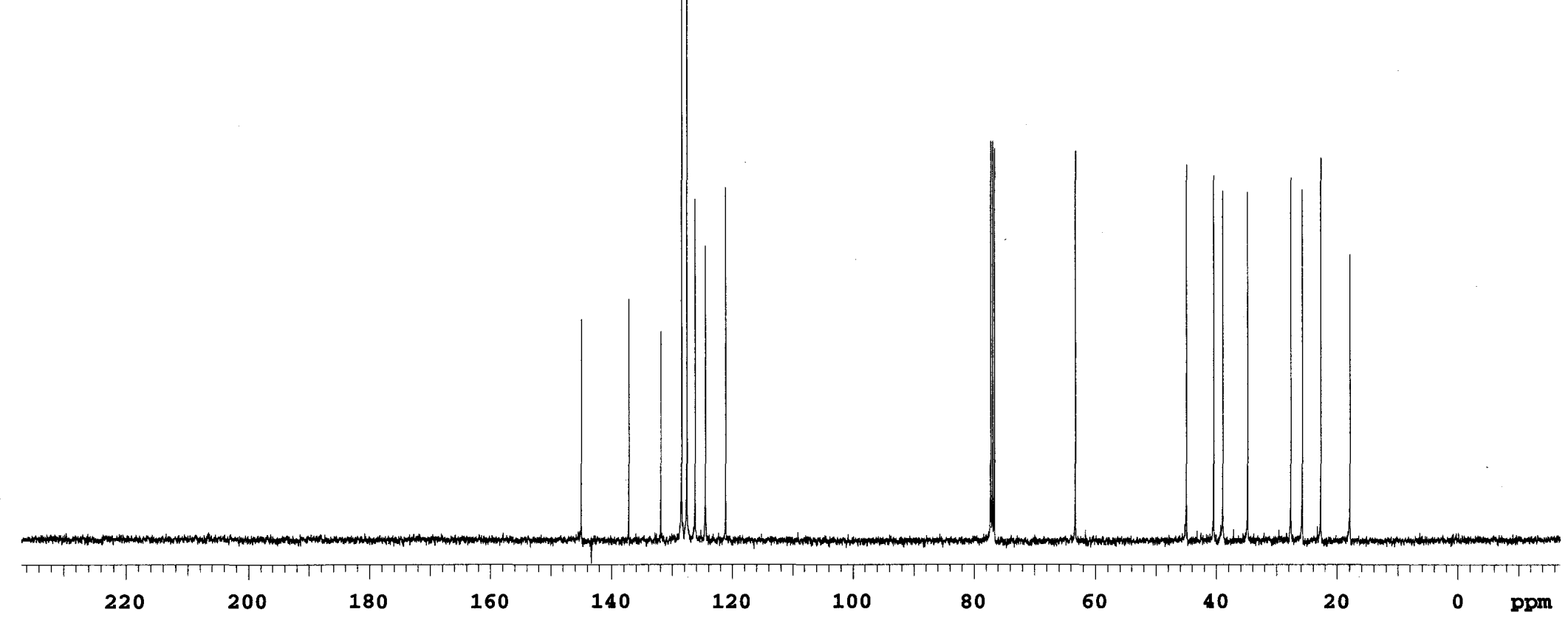




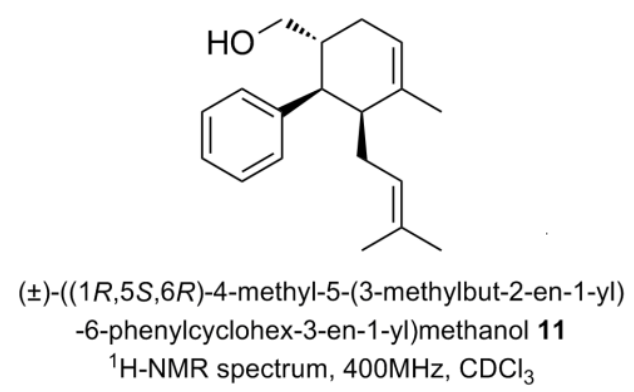

${ }^{1} \mathrm{H}$-NMR spectrum, $400 \mathrm{MHz}, \mathrm{CDCl}_{3}$

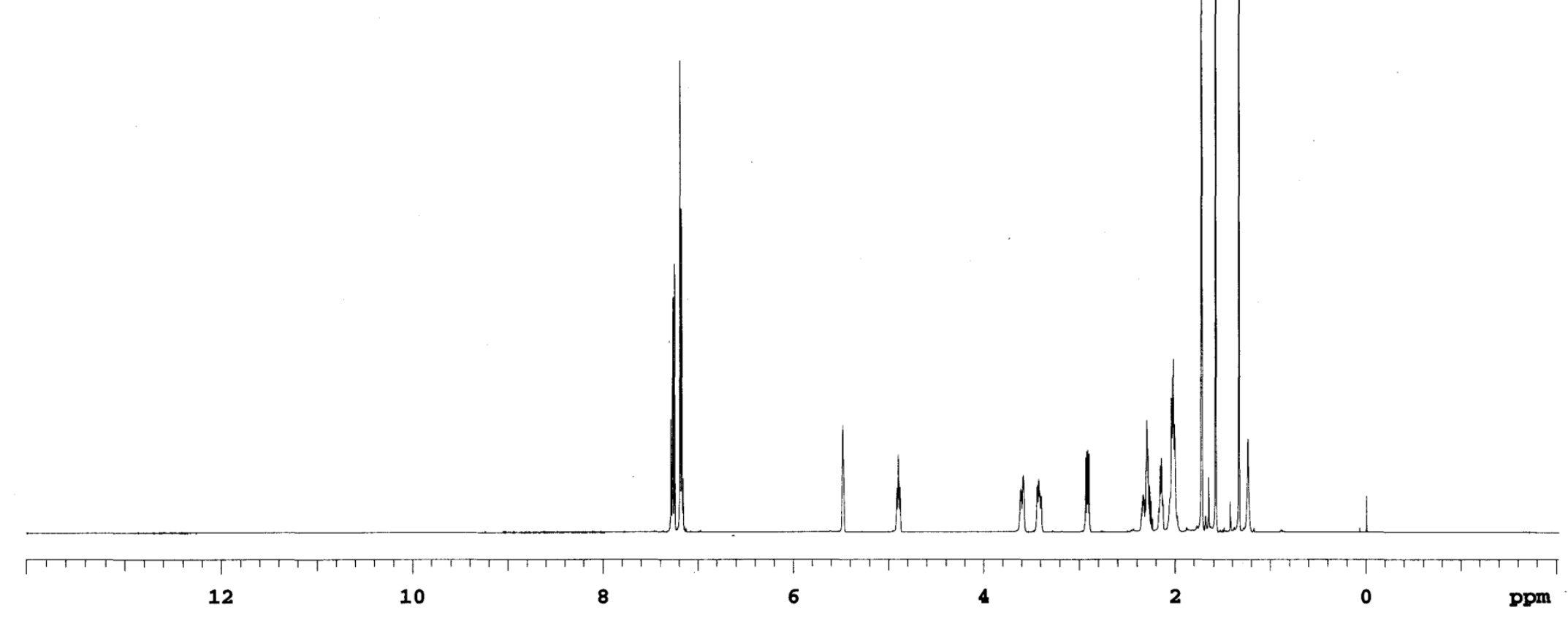




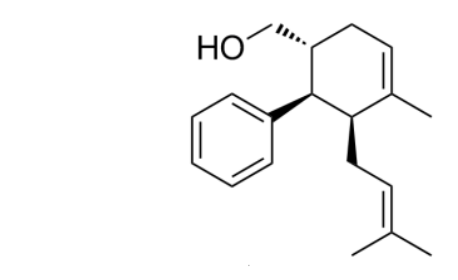

$( \pm)-((1 R, 5 S, 6 R)-4-m e t h y l-5-(3-m e t h y l b u t-2-e n-1-y l)$

-6-phenylcyclohex-3-en-1-yl)methanol 11

${ }^{13} \mathrm{C}$-NMR spectrum, $100 \mathrm{MHz}, \mathrm{CDCl}_{3}$

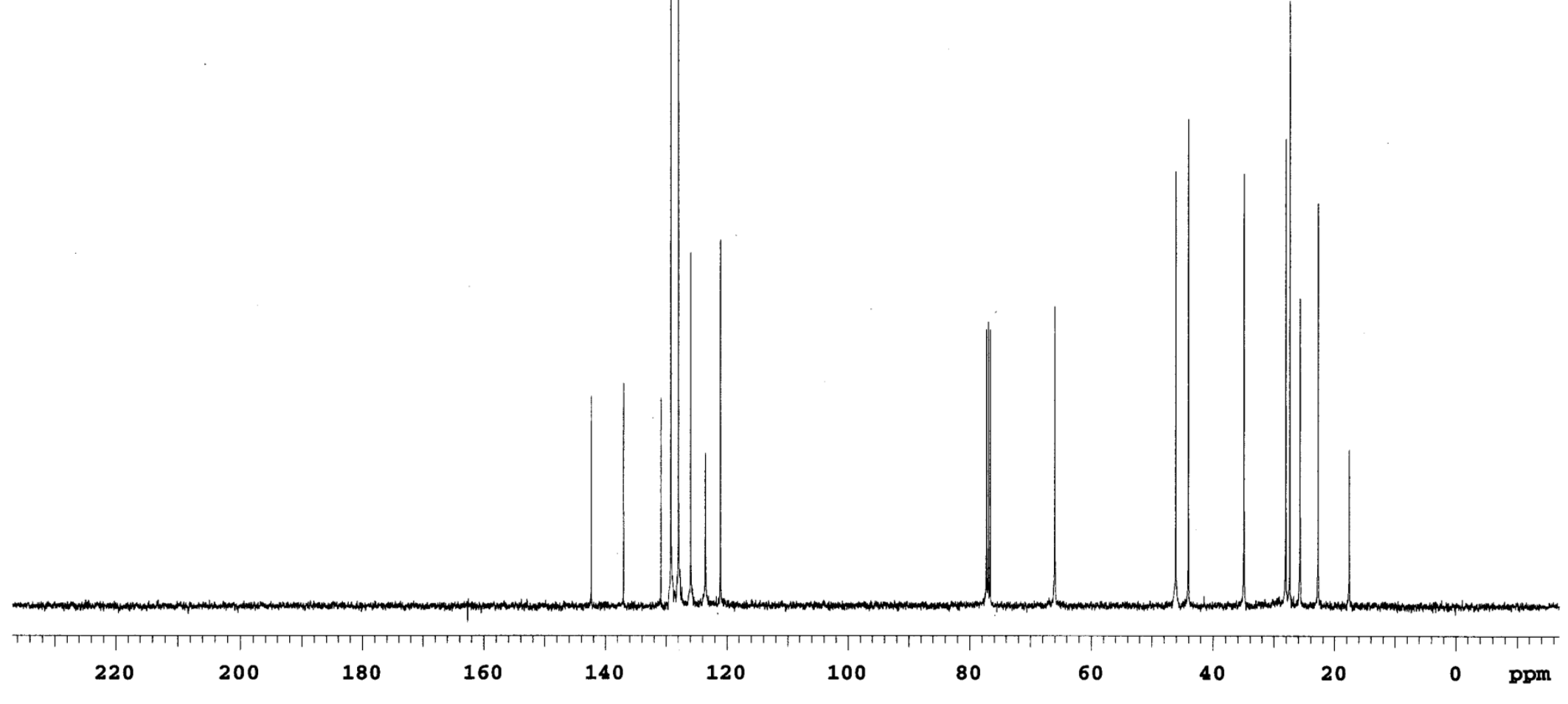




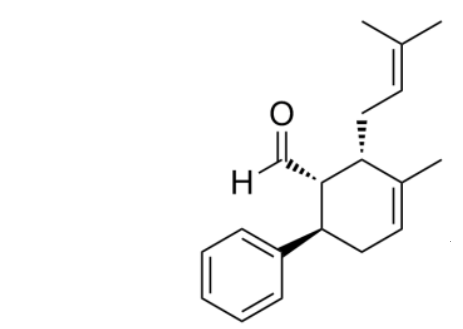

$( \pm)-((1 R, 2 S, 6 R)-3-m e t h y l-2-(3-m e t h y l b u t-2-e n-1-y l)$

-6-phenylcyclohex-3-en-1-yl)methanal 12

${ }^{1} \mathrm{H}-\mathrm{NMR}$ spectrum, $400 \mathrm{MHz}, \mathrm{CDCl}_{3}$

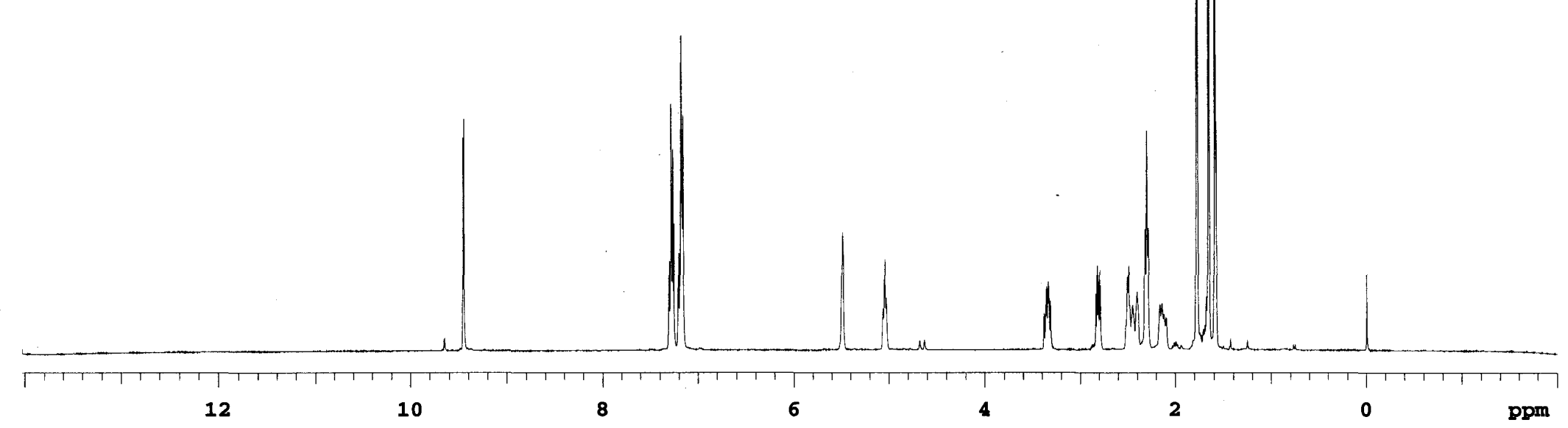




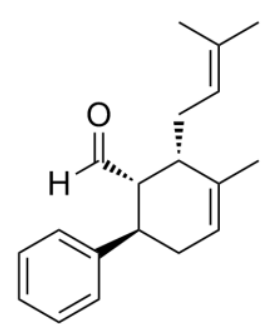

$( \pm)-((1 R, 2 S, 6 R)-3$-methyl-2-(3-methylbut-2-en-1-yl) -6-phenylcyclohex-3-en-1-yl)methanal 12

${ }^{13} \mathrm{C}$-NMR spectrum, $100 \mathrm{MHz}, \mathrm{CDCl}_{3}$

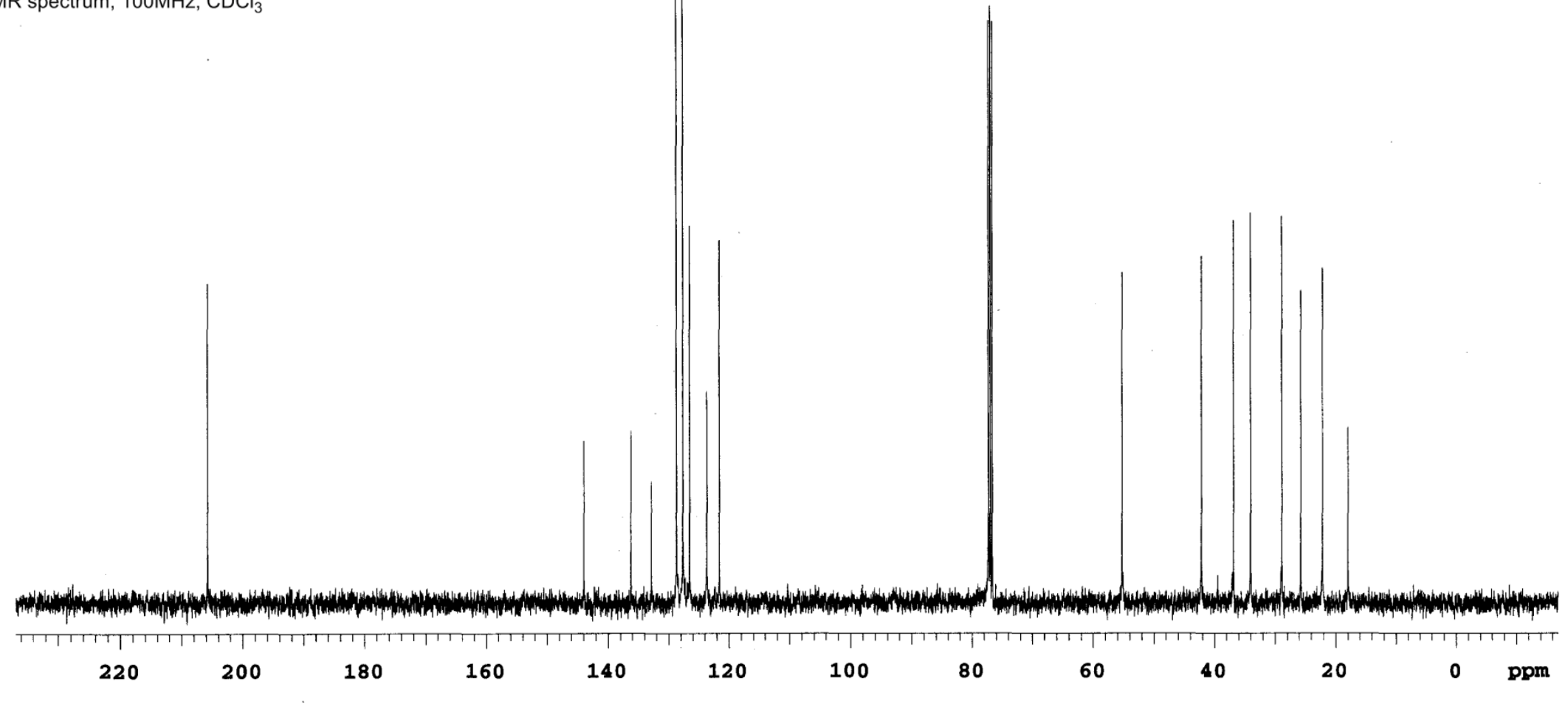




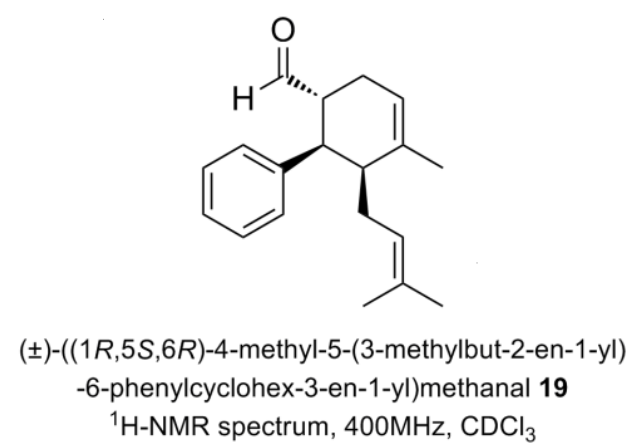

${ }^{1} \mathrm{H}$-NMR spectrum, $400 \mathrm{MHz}, \mathrm{CDCl}_{3}$ 


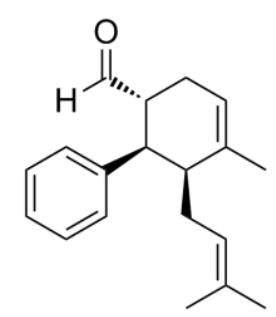

( \pm )-((1R,5S,6R)-4-methyl-5-(3-methylbut-2-en-1-yl) -6-phenylcyclohex-3-en-1-yl)methanal 19

${ }^{13} \mathrm{C}$-NMR spectrum, $100 \mathrm{MHz}, \mathrm{CDCl}_{3}$

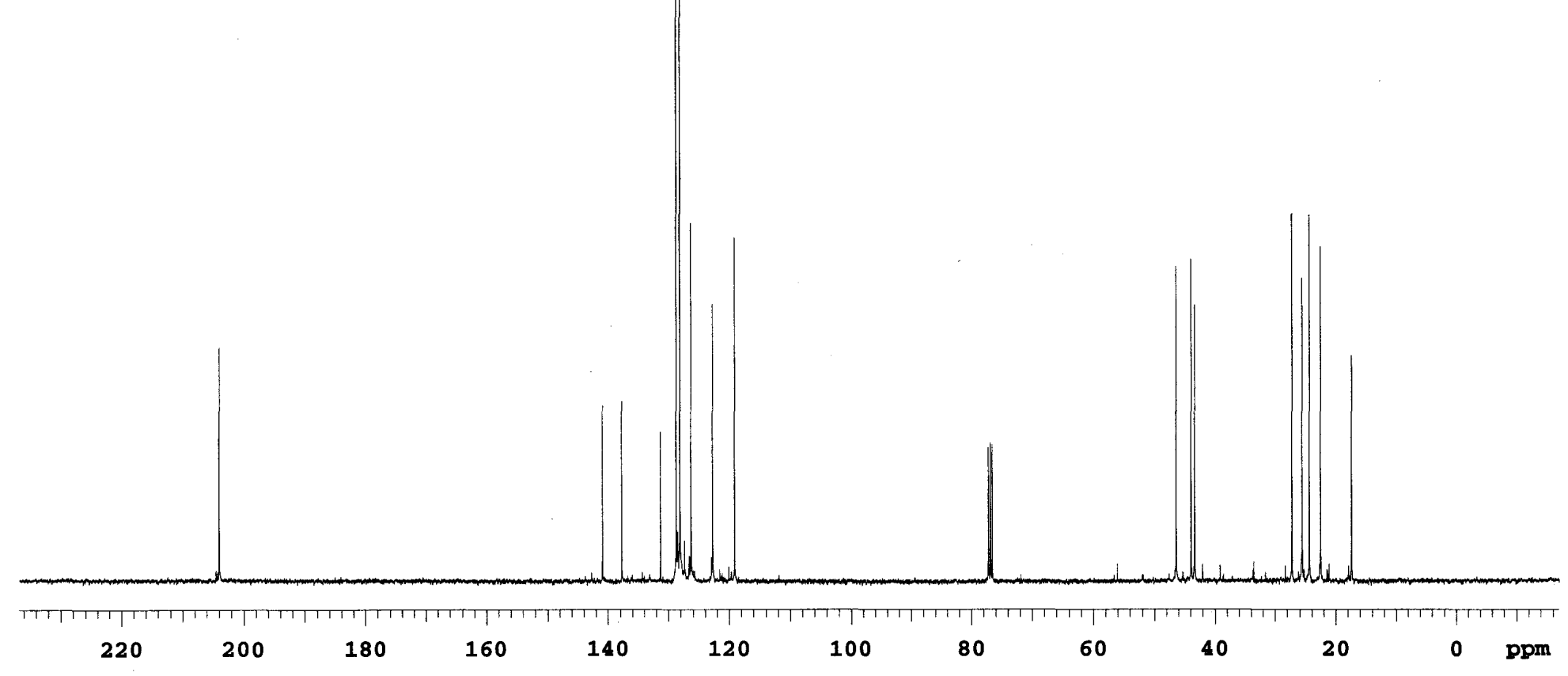




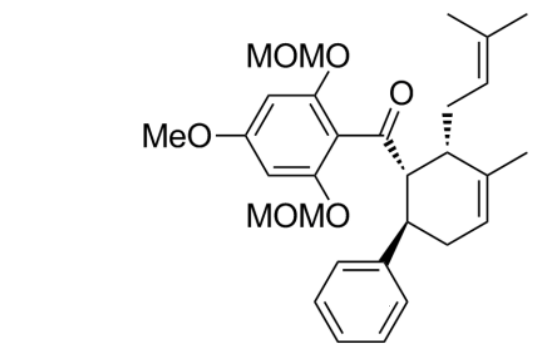

( \pm )-((1R,2S,6R)-3-methyl-2-(3-methylbut-2-en-1-yl)-6phenylcyclohex-3-en-1-yl) (4-methoxy-2,6-

bis(methoxymethoxy) phenyl) methanone 14 ${ }^{1} \mathrm{H}-\mathrm{NMR}$ spectrum, $400 \mathrm{MHz}, \mathrm{CDCl}_{3}$

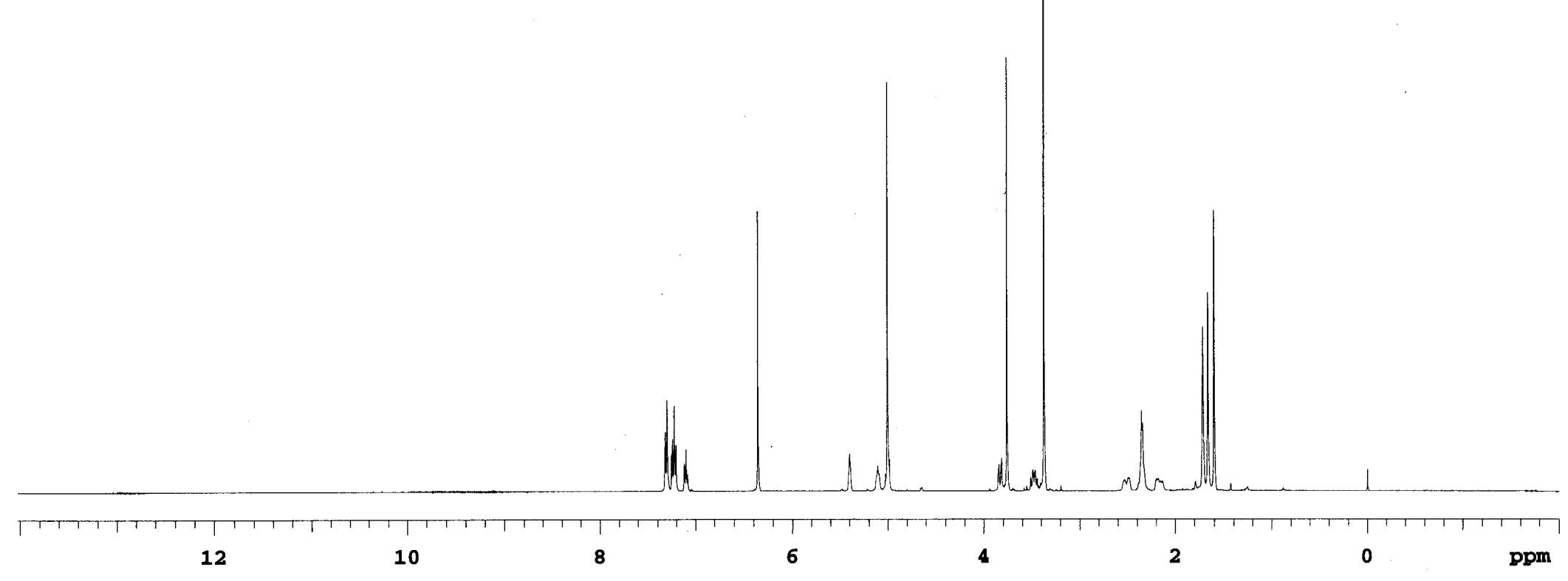




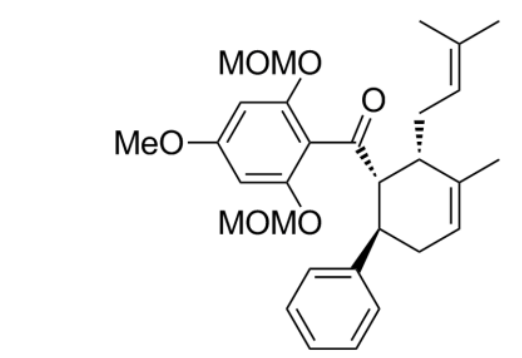

$( \pm)-((1 R, 2 S, 6 R)-3-m e t h y l-2-(3-m e t h y l b u t-2-e n-1-y \mid)-6-$ phenylcyclohex-3-en-1-yl) (4-methoxy-2,6bis(methoxymethoxy) phenyl) methanone 14 ${ }^{13} \mathrm{C}-\mathrm{NMR}$ spectrum, $100 \mathrm{MHz}, \mathrm{CDCl}_{3}$

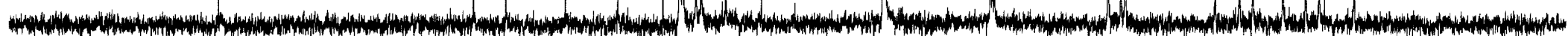

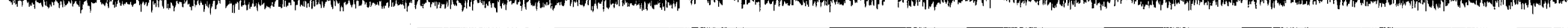

200

180

160

140

120

100

80

60

40

20

0 ppm 


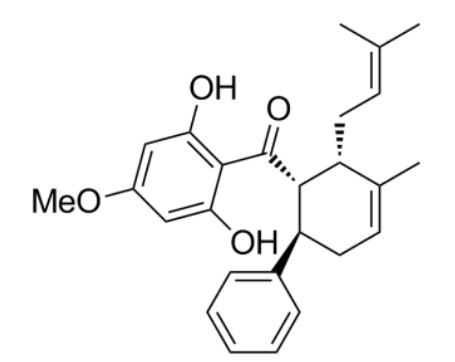

panduratin A 1

${ }^{1} \mathrm{H}-\mathrm{NMR}$ spectrum, $400 \mathrm{MHz}, \mathrm{CD}_{3} \mathrm{OD}$

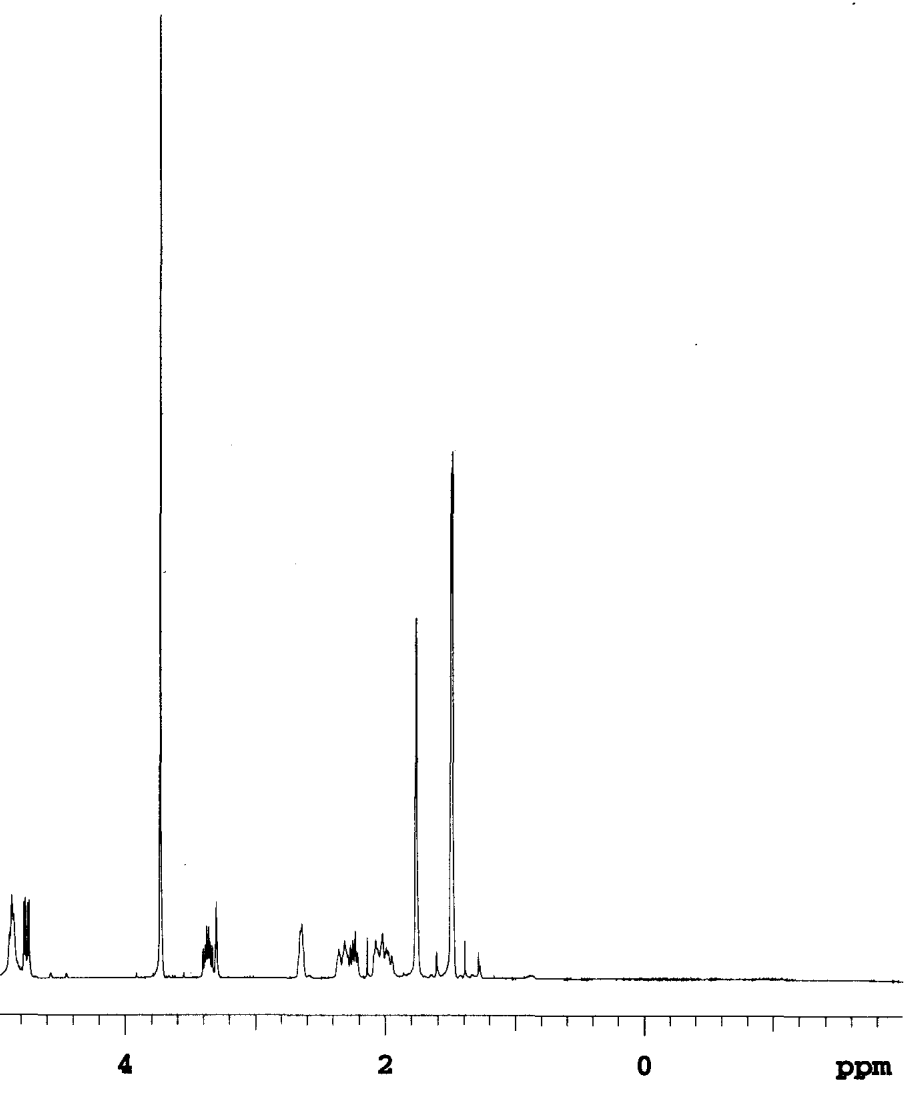




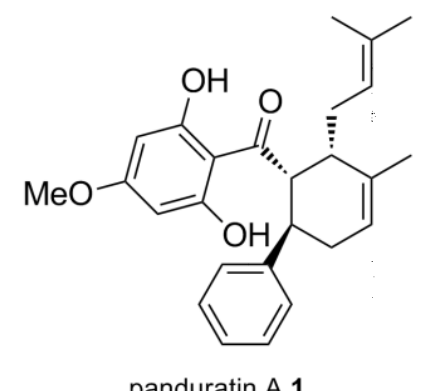

${ }^{13} \mathrm{C}$-NMR spectrum, $100 \mathrm{MHz}, \mathrm{CD}_{3} \mathrm{OD}$

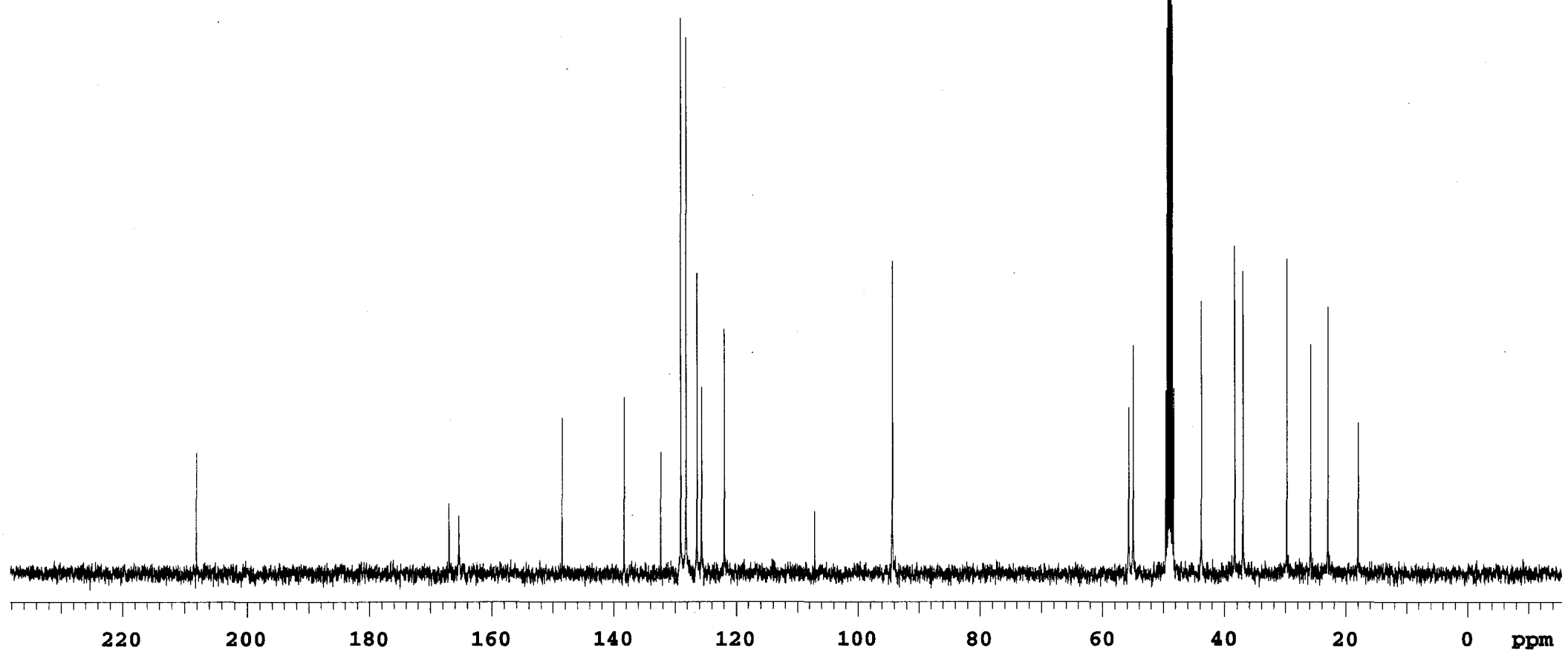




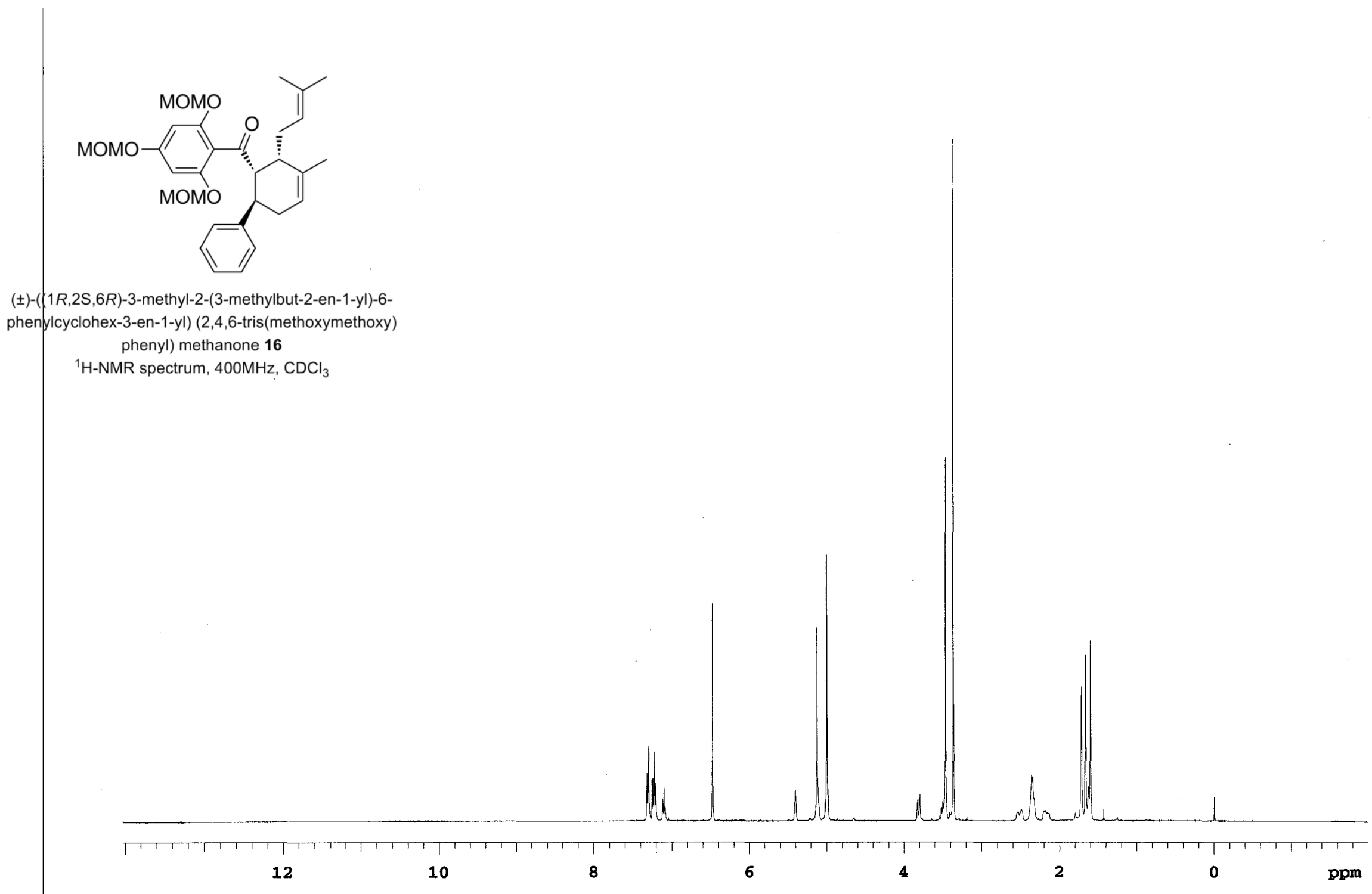




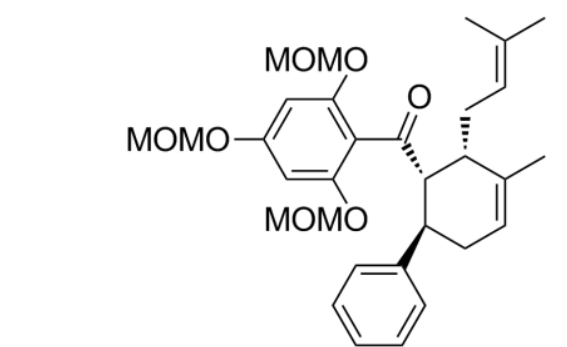

$( \pm)-((1 R, 2 \mathrm{~S}, 6 R)-3-$ methyl-2-(3-methylbut-2-en-1-yl)-6phenylcyclohex-3-en-1-yl) (2,4,6-tris(methoxymethoxy) phenyl) methanone 16

${ }^{13} \mathrm{C}$-NMR spectrum, $100 \mathrm{MHz}, \mathrm{CDCl}_{3}$

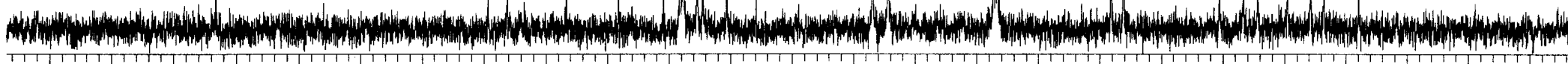




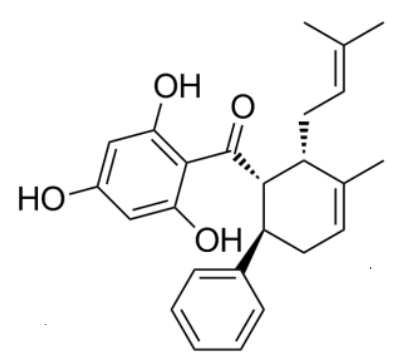

4-hydroxypanduratin $\mathrm{A}$

${ }^{1} \mathrm{H}$-NMR spectrum, $400 \mathrm{MHz}, \mathrm{CD}_{3} \mathrm{OD}$

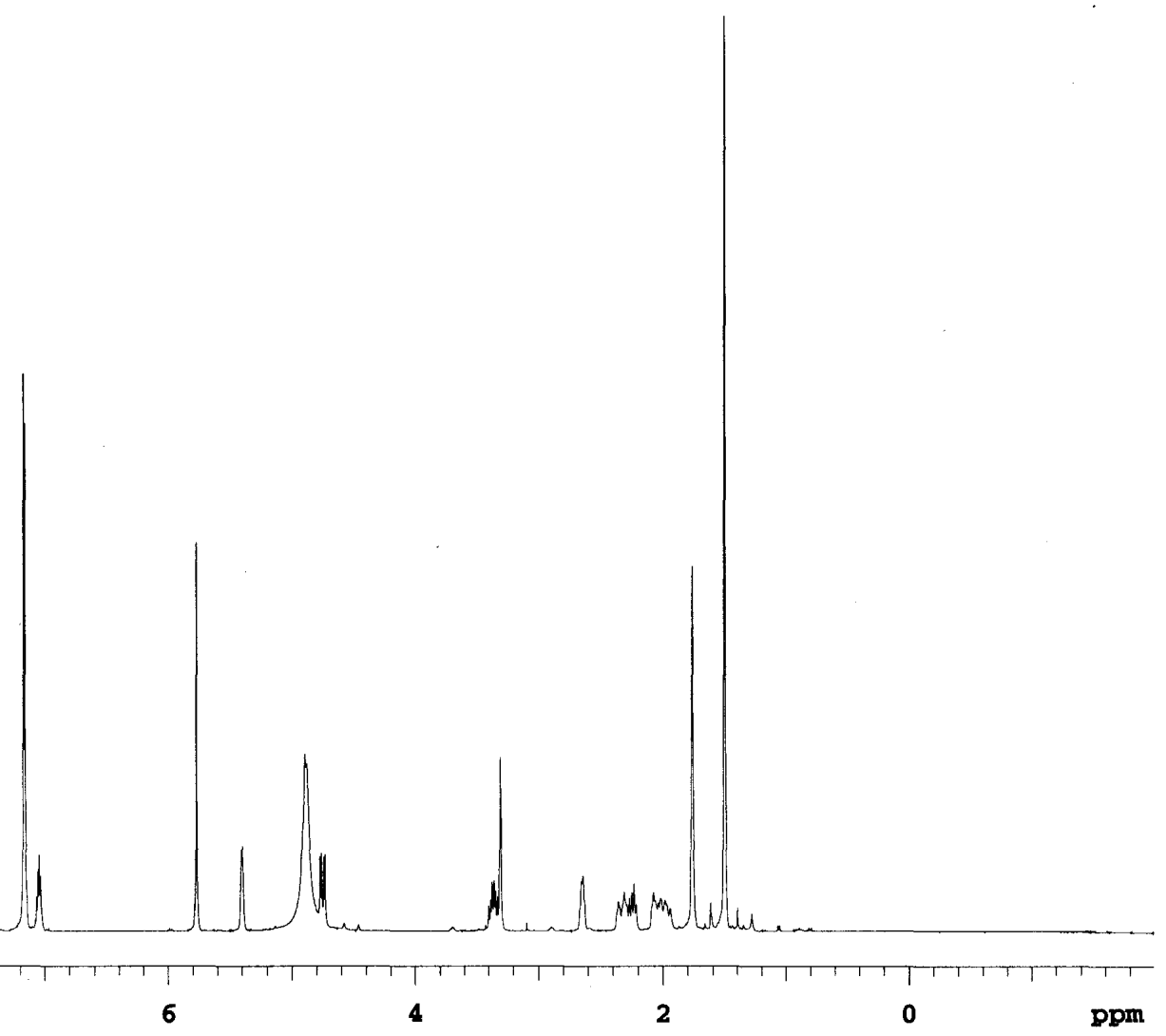




$$
11
$$




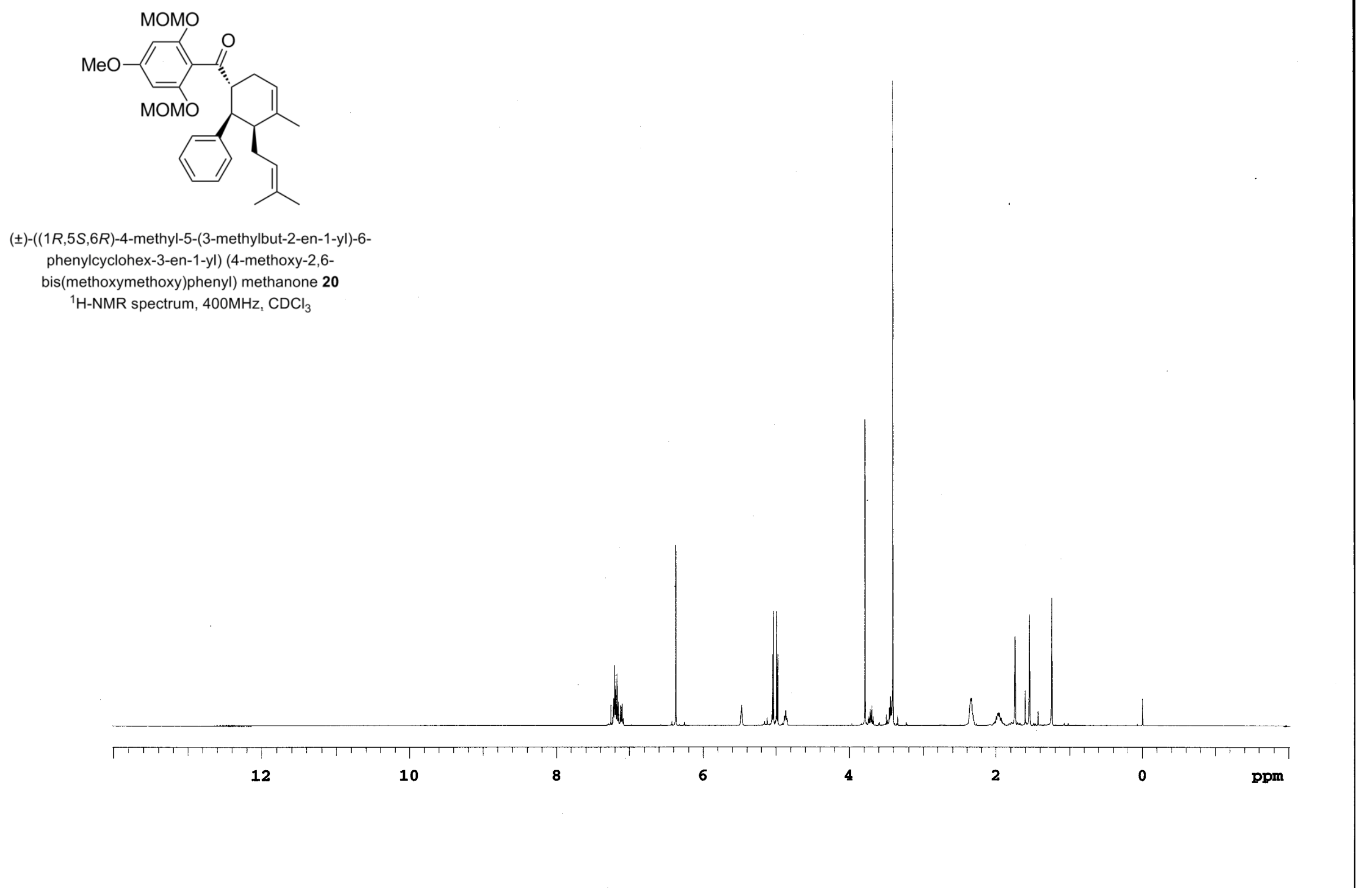




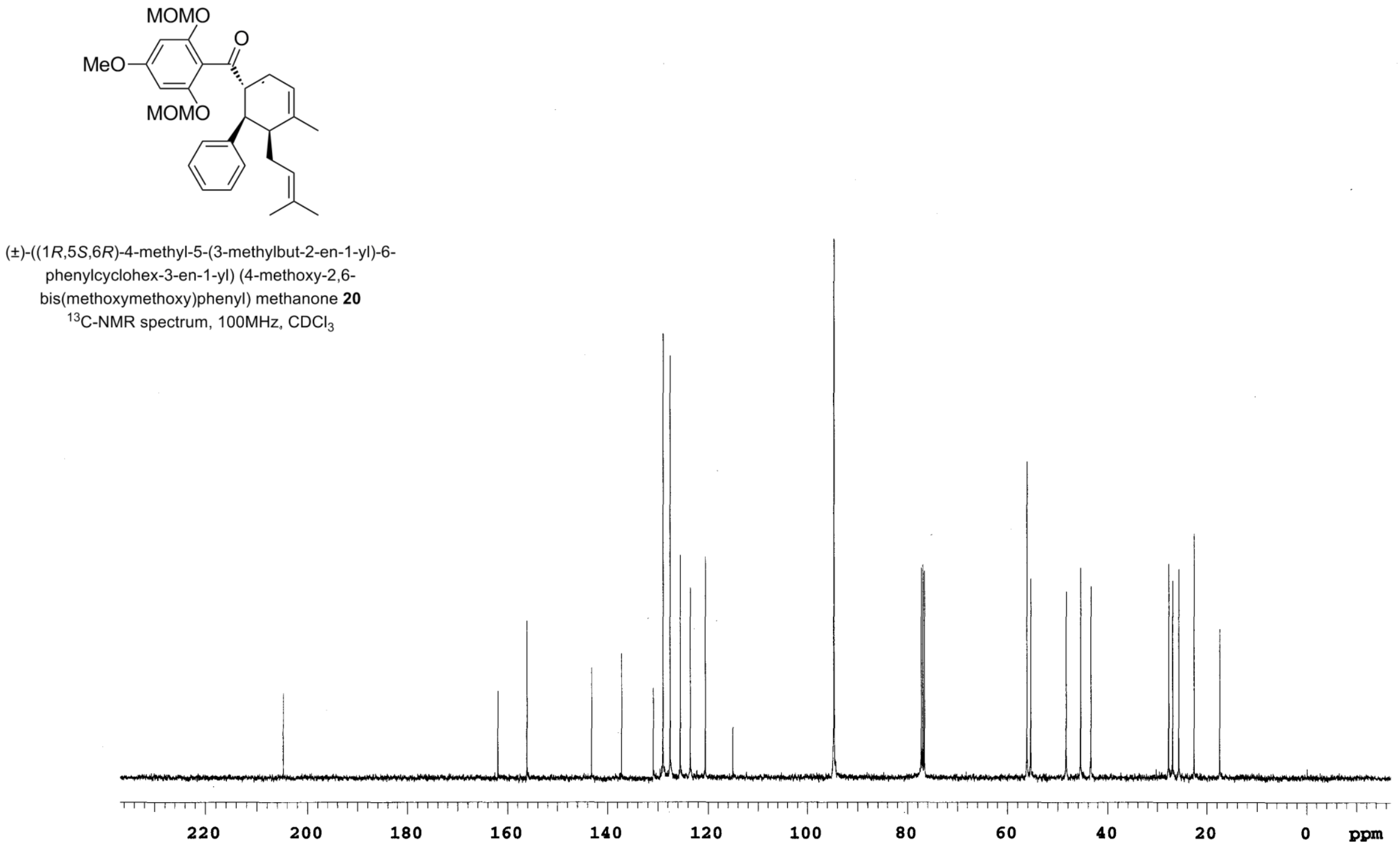




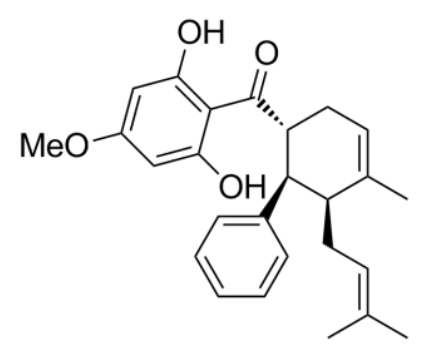

nicolaioidesin B $\mathbf{5}$

${ }^{1} \mathrm{H}-\mathrm{NMR}$ spectrum, $400 \mathrm{MHz}, \mathrm{CD}_{3} \mathrm{OD}$

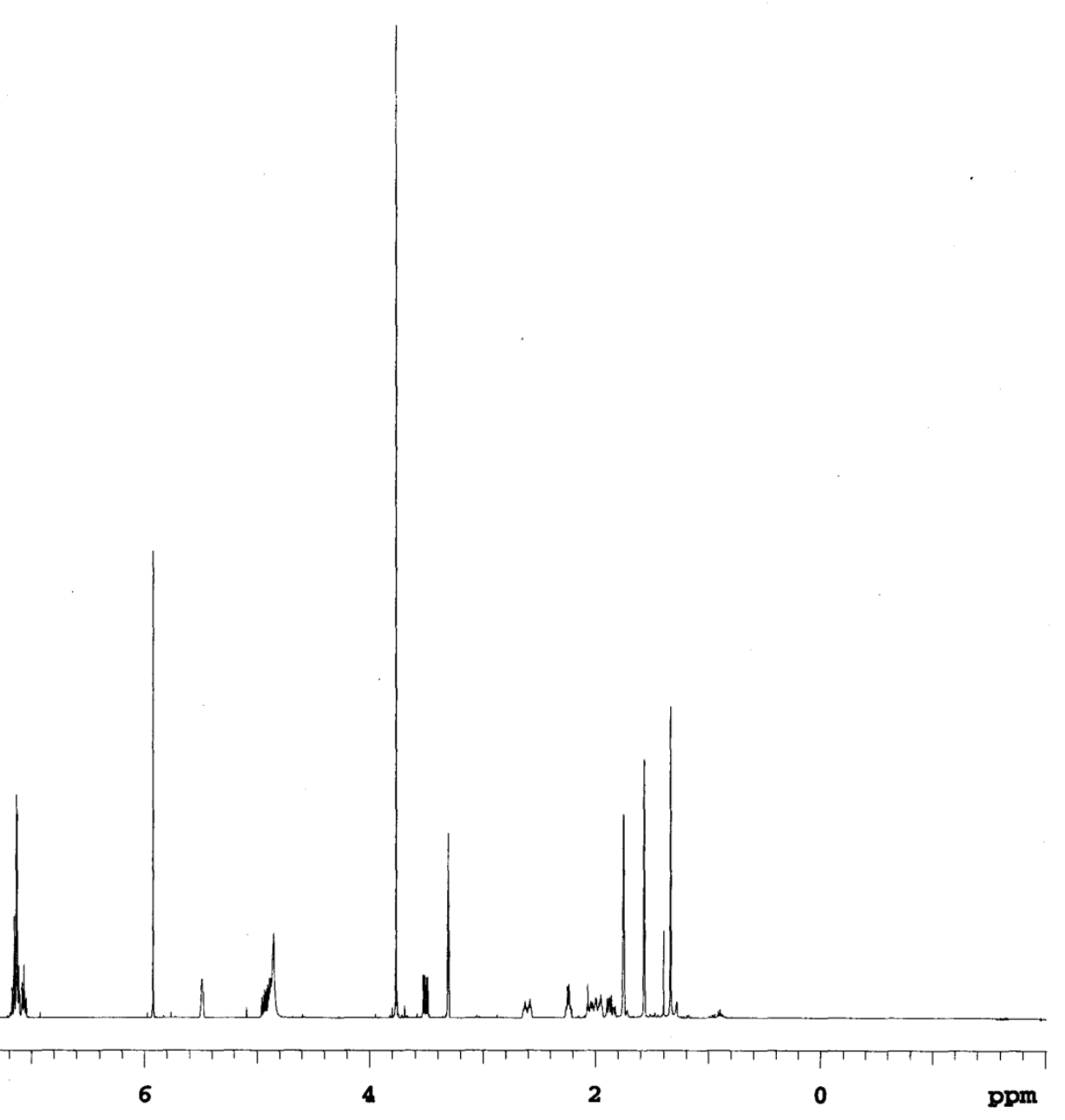




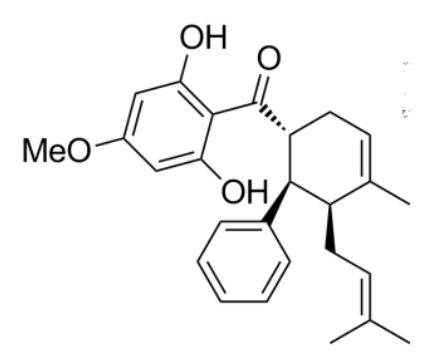

nicolaioidesin B $\mathbf{5}$

${ }^{13} \mathrm{C}-\mathrm{NMR}$ spectrum, $100 \mathrm{MHz}, \mathrm{CD}_{3} \mathrm{OD}$

whom

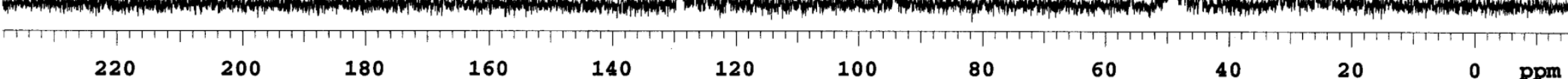




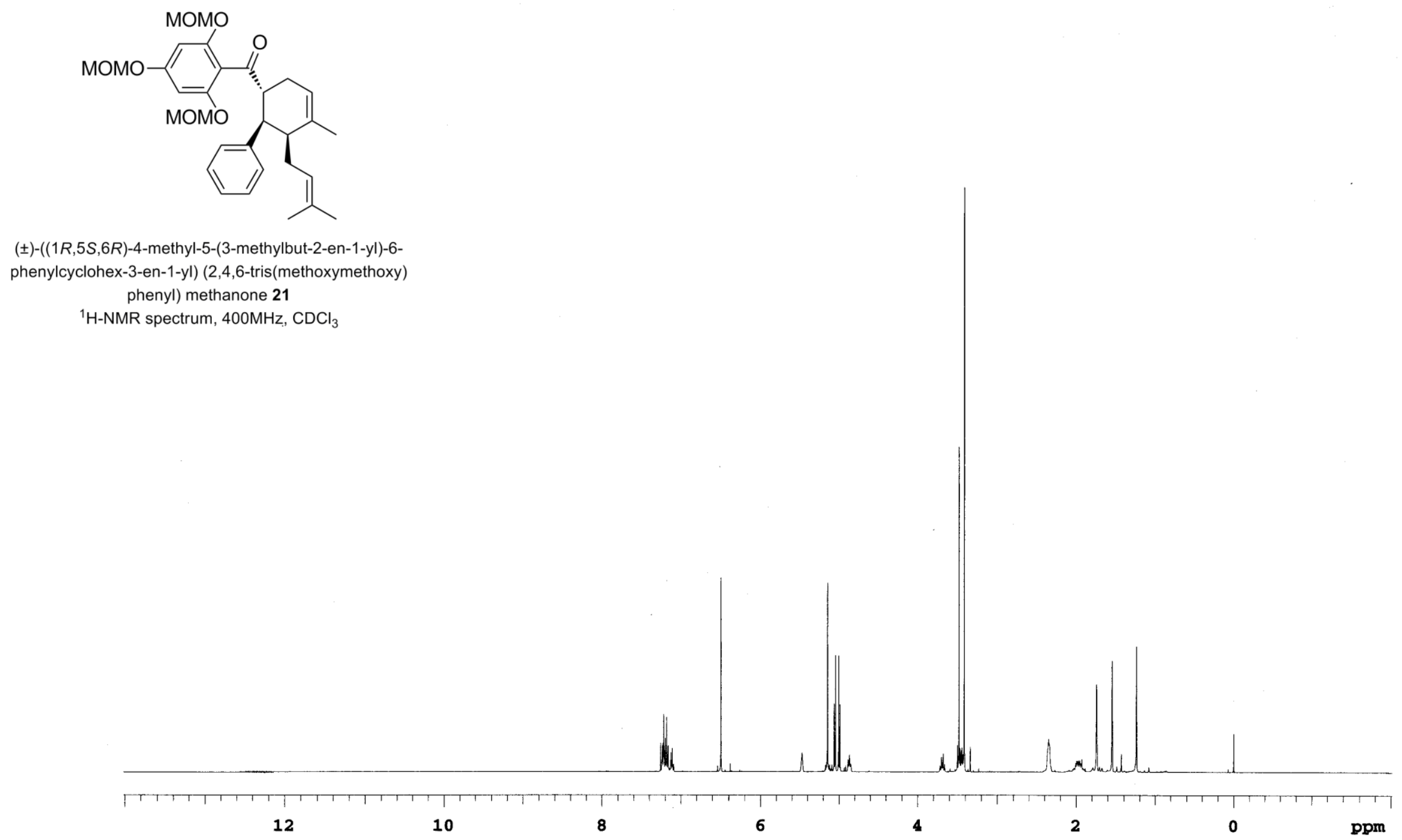




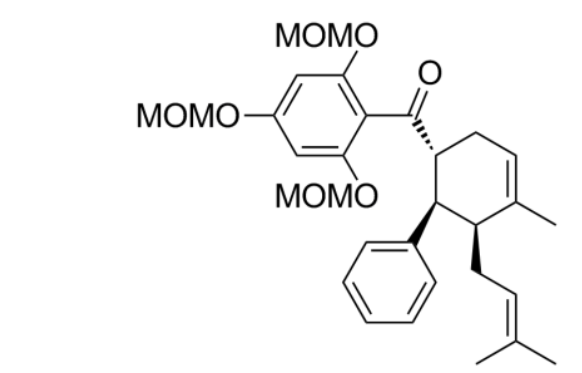

$( \pm)-((1 R, 5 S, 6 R)-4-m e t h y l-5-(3-m e t h y l b u t-2-e n-1-y l)-6-$ phenylcyclohex-3-en-1-yl) (2,4,6-tris(methoxymethoxy) phenyl) methanone 21

${ }^{13} \mathrm{C}$-NMR spectrum, $100 \mathrm{MHz}, \mathrm{CDCl}_{3}$

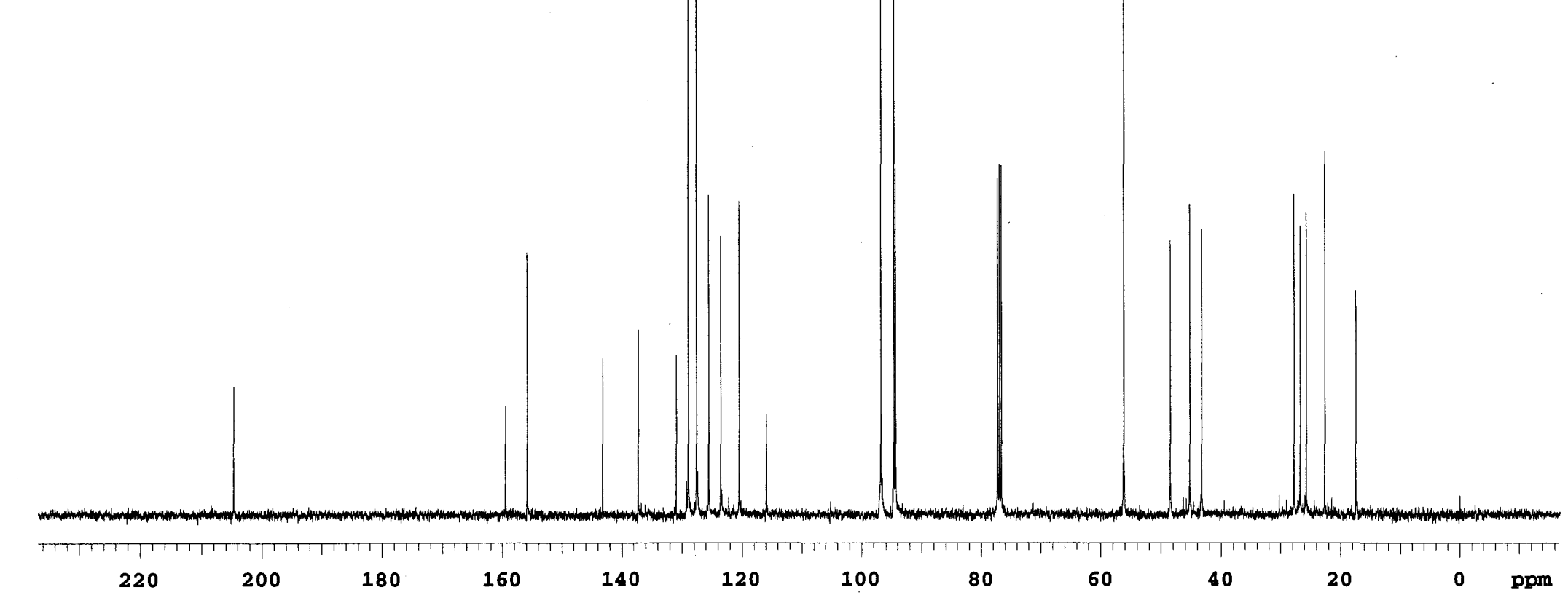




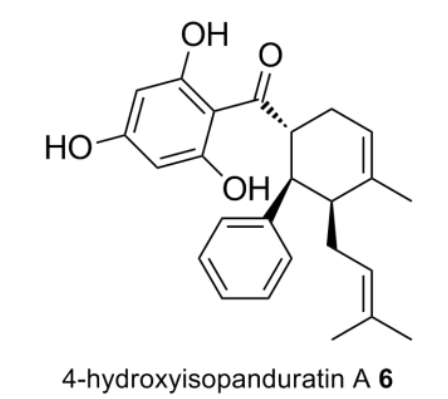

${ }^{1} \mathrm{H}-\mathrm{NMR}$ spectrum, $400 \mathrm{MHz}, \mathrm{CD}_{3} \mathrm{OD}$

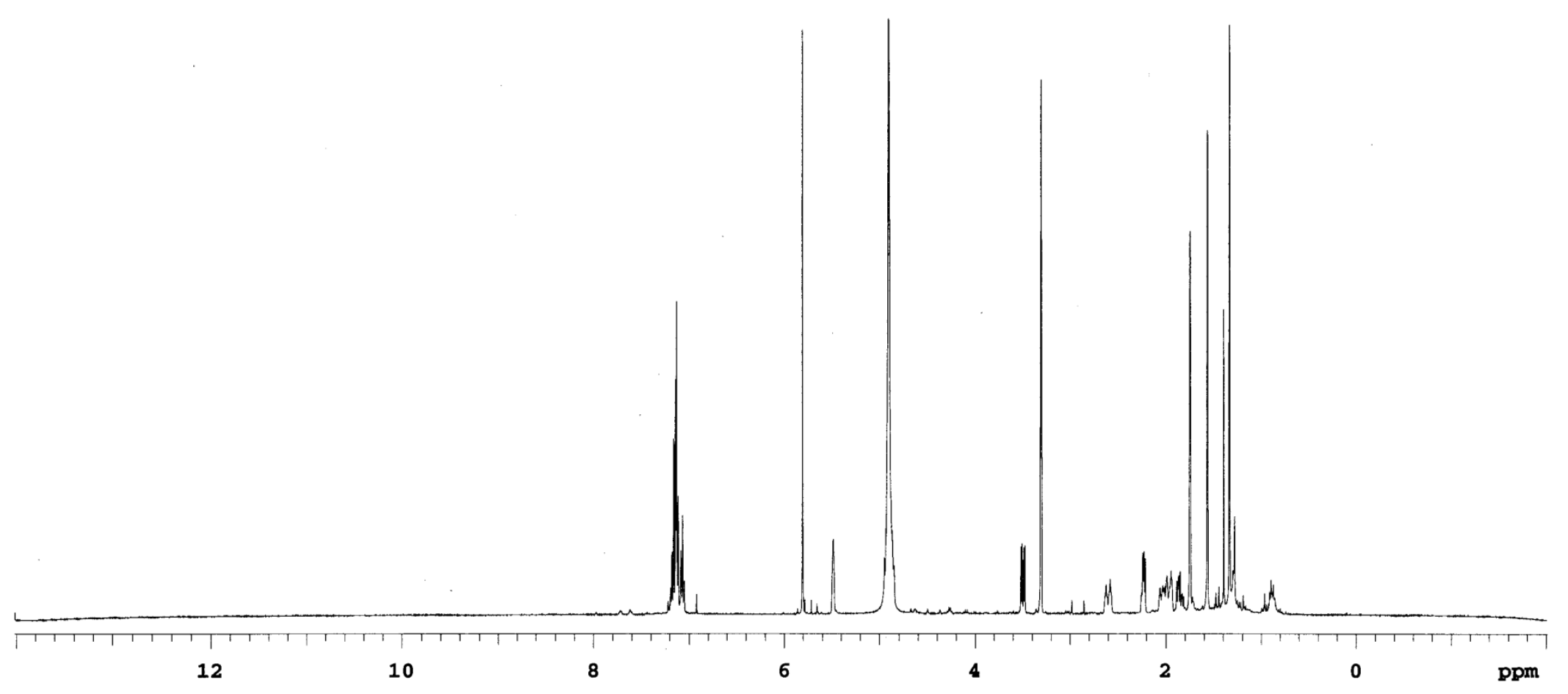




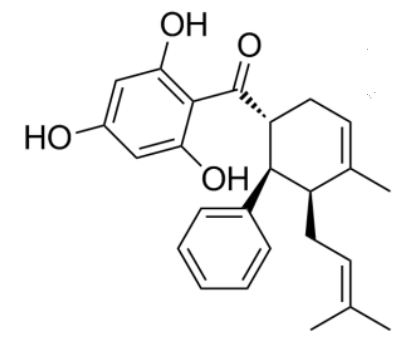

4-hydroxyisopanduratin $\mathrm{A} 6$

${ }^{13} \mathrm{C}$-NMR spectrum, $100 \mathrm{MHz}, \mathrm{CD}_{3} \mathrm{OD}$

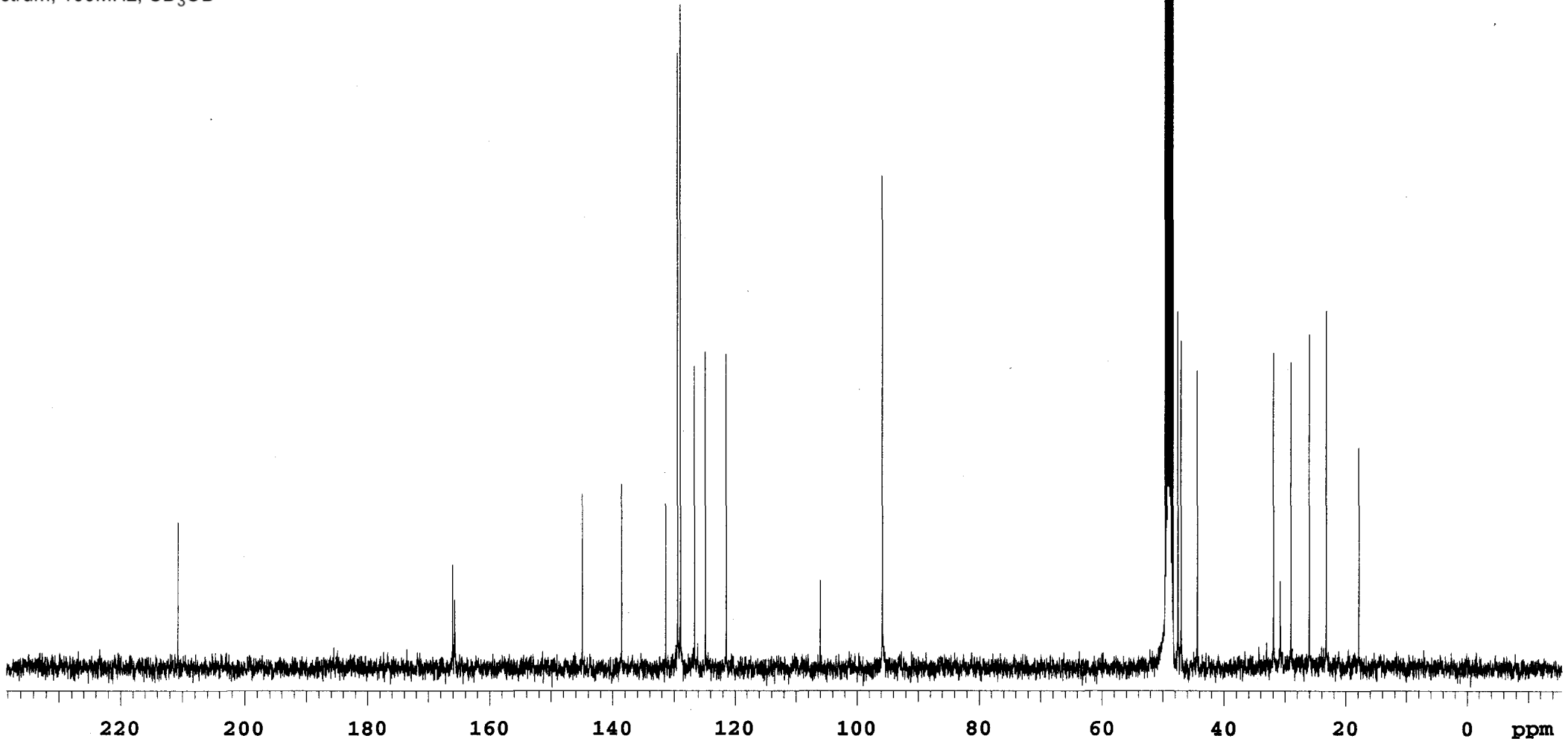

